Supporting Information for

\title{
Synthesis of Difluorinated Halohydrins by the Chemoselective Addition of Difluoroenolates to $\alpha$-Haloketones
}

\author{
Munia F. Sowaileh, Maali D. Alshammari, and David A. Colby* \\ Department of BioMolecular Sciences, University of Mississippi, University, Mississippi 38677
}

\section{Table of Contents:}

I. Experimental and Characterization Data.........................................S1

II. References............................................................................. S11

III. Spectral Data............................................................................

\section{Experimental and Characterization Data}

General Procedures. Experiments requiring anhydrous conditions were performed under argon atmosphere and organic solvents were dried over molecular sieves. All solvents and reagents were purchased from commercial sources unless otherwise noted. Thin-layer chromatography was conducted using MilliporeSigma TLC silica gel $60 \mathrm{~F}_{254}$ plates. Preparative thin-layer chromatography was performed using Sorbent Technologies silica G prep TLC plates with UV254. Flash chromatography was conducted using SiliCycle Siliaflash silica gel P60 (40-63 $\mu \mathrm{m}) 60 \AA$. Melting points were taken on an OptiMelt apparatus from Stanford Research Systems and are not corrected. NMR spectra were recorded on a Bruker ARX $300 \mathrm{MHz}$, a Bruker Topspin Avance III HD $500 \mathrm{MHz}$ spectrometer equipped with prodigy cryoprobe, or a Bruker Avance III HD 400 $\mathrm{MHz}$ spectrometer. The residual solvent peaks for chloroform were used as an internal standard for ${ }^{1} \mathrm{H}$ and ${ }^{13} \mathrm{C}$ NMR spectra (i.e., $7.26 \mathrm{ppm}$ and $77.16 \mathrm{ppm}$, respectively) whereas trifluorotoluene was used as an added internal standard for ${ }^{19} \mathrm{~F}$ NMR spectra (i.e., $-63.72 \mathrm{ppm}$ ). Mass spectrometry were acquired by the Department of Chemistry at the University of Mississippi using SYNAPT HD Mass Spectrometer from Waters. Infrared spectra were recorded on Agilent Technologies Cary 630 FTIR. The $\alpha$-haloketones were purchased from commercial suppliers and used without further purification. The pentafluoro-gem-diols were synthesized according to the reported procedures. ${ }^{1,2}$

Table S1. Optimization of the Preparation of Halohydrin 1. ${ }^{3}$

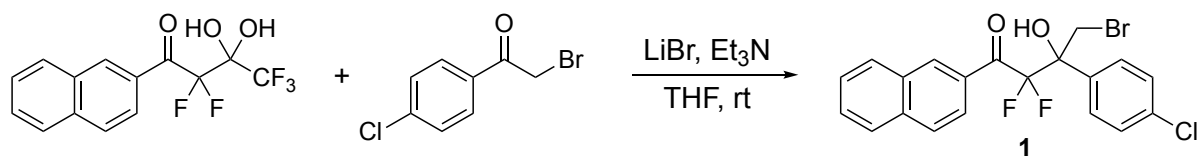

$\begin{array}{cccccc}\text { entry } & \begin{array}{c}\text { haloketone } \\ \text { (equiv.) }\end{array} & \mathrm{Et}_{3} \mathrm{~N} \text { (equiv.) } & \text { LiBr (equiv.) } & \text { conc. (M) } & \text { yield }\left({ }^{19} \mathrm{~F} \mathrm{NMR}\right) \\ 1 & 1 & 1.2 & - & 0.1 & 0 \% \\ 2 & 1 & 1.2 & 5 & 0.1 & 54 \% \\ 3 & 2 & 1.2 & 5 & 0.1 & 77 \%\end{array}$




$\begin{array}{cccccc}4 & 2 & 1.2 & 5 & 0.5 & 87 \% \\ 5 & 2 & 3 & 5 & 0.5 & 65 \%\end{array}$

Representative Procedure for the Preparation of Halohydrins. ${ }^{3}$ To a solution of 2,2,4,4,4pentafluoro-3,3-dihydroxy-1-(naphthalen-2-yl)-butan-1-one ${ }^{1} \quad(100 \quad \mathrm{mg}, \quad 0.3 \quad \mathrm{mmol}), \quad 2-$ bromoacetophenone (124 mg, $0.624 \mathrm{mmol})$, and $\mathrm{LiBr}(135 \mathrm{mg}, 1.56 \mathrm{mmol})$ in THF (500 $\mu \mathrm{L})$ was added $\mathrm{Et}_{3} \mathrm{~N}(53 \mu \mathrm{L}, 0.37 \mathrm{mmol})$ dropwise at $0{ }^{\circ} \mathrm{C}$. The mixture was warmed to $\mathrm{rt}$ and stirred for 30 min. The mixture was quenched with saturated aqueous $\mathrm{NH}_{4} \mathrm{Cl}(3 \mathrm{~mL})$ and extracted with EtOAc $(3 \times 5 \mathrm{~mL})$. The organics were dried over $\mathrm{Na}_{2} \mathrm{SO}_{4}$ and concentrated under reduced pressure. $\mathrm{SiO}_{2}$ flash chromatography (19:1 hexanes/EtOAc) afforded the product $\mathbf{1}$ in $88 \%$ yield (98 $\mathrm{mg})$ as a colorless solid.

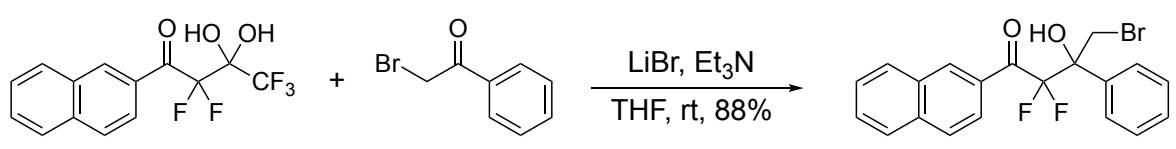

1

4-Bromo-2,2-difluoro-3-hydroxy-1-(naphthalen-2-yl)-3-phenylbutan-1-one $1 . \quad$ See representative reaction procedure: $\mathrm{mp} 64-65{ }^{\circ} \mathrm{C} ;{ }^{1} \mathrm{H} \mathrm{NMR}\left(400 \mathrm{MHz}, \mathrm{CDCl}_{3}\right) \delta 8.54(\mathrm{~s}, 1 \mathrm{H}), 7.95$ $(\mathrm{dd}, J=14.2,8.5 \mathrm{~Hz}, 2 \mathrm{H}), 7.85(\mathrm{dd}, J=8.4,4.6 \mathrm{~Hz}, 2 \mathrm{H}), 7.63(\mathrm{ddd}, J=8.3,6.9,1.3 \mathrm{~Hz}, 1 \mathrm{H})$, 7.60-7.53 (m, 3H), 7.43-7.36 (m, 3H), $4.26(\mathrm{~s}, 2 \mathrm{H}), 3.53(\mathrm{~s}, 1 \mathrm{H}) ;{ }^{13} \mathrm{C} \mathrm{NMR}\left(100 \mathrm{MHz}, \mathrm{CDCl}_{3}\right) \delta$ $190.4\left(\mathrm{dd}, J_{\mathrm{CF}}=29.9,27.7 \mathrm{~Hz}, 1 \mathrm{C}\right), 136.4,135.9,133.5\left(\mathrm{dd}, J_{\mathrm{CF}}=5.9,4.0 \mathrm{~Hz}, 1 \mathrm{C}\right), 132.0,130.4$ $\left(\mathrm{dd}, J_{\mathrm{CF}}=2.6,1.1 \mathrm{~Hz}, 1 \mathrm{C}\right), 130.2,129.4,128.9,128.4(2 \mathrm{C}), 128.1,127.6,126.9,126.8\left(\mathrm{dd}, J_{\mathrm{CF}}=\right.$ $3.3,1.5 \mathrm{~Hz}, 2 \mathrm{C}), 125.0\left(\mathrm{dd}, J_{\mathrm{CF}}=2.9,2.2 \mathrm{~Hz}, 1 \mathrm{C}\right), 117.6\left(\mathrm{q}, J_{\mathrm{CF}}=262.4 \mathrm{~Hz}, 1 \mathrm{C}\right), 78.0\left(\mathrm{t}, J_{\mathrm{CF}}=\right.$ $24.8 \mathrm{~Hz}, 1 \mathrm{C}), 37.8\left(\mathrm{t}, J_{\mathrm{CF}}=3.5 \mathrm{~Hz}, 1 \mathrm{C}\right) ;{ }^{19} \mathrm{~F} \mathrm{NMR}\left(376 \mathrm{MHz}, \mathrm{CDCl}_{3}\right) \delta-106.0\left(\mathrm{~d}, J_{F F}=270.6 \mathrm{~Hz}\right.$, $1 \mathrm{~F}),-107.4\left(\mathrm{~d}, J_{F F}=270.7 \mathrm{~Hz}, 1 \mathrm{~F}\right)$; IR (film) $v_{\max } 3519,3061,2925,1688,1626,1450 \mathrm{~cm}^{-1}$; HRMS (ESI-TOF) $m / z$ calcd for $\mathrm{C}_{20} \mathrm{H}_{16} \mathrm{BrF}_{2} \mathrm{O}_{2}(\mathrm{M}+\mathrm{H})^{+} 405.0302$, found 405.0306.

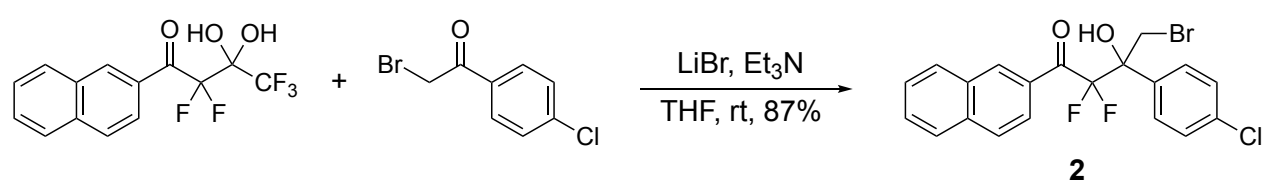

4-Bromo-3-(4-chlorophenyl)-2,2-difluoro-3-hydroxy-1-(naphthalen-2-yl)butan-1-one 2. See representative reaction procedure. A solution of 2,2,4,4,4-pentafluoro-3,3-dihydroxy-1(naphthalen-2-yl)-butan-1-one ${ }^{1}(300 \mathrm{mg}, 1.0 \mathrm{mmol}), 2$-bromo-4'-chloroacetophenone (438 $\mathrm{mg}$, $1.87 \mathrm{mmol})$, and $\mathrm{LiBr}(244 \mathrm{mg}, 2.81 \mathrm{mmol})$ in THF $(2 \mathrm{~mL})$ was treated with $\mathrm{Et}_{3} \mathrm{~N}(157 \mu \mathrm{L}, 1.12$ mmol). $\mathrm{SiO}_{2}$ flash chromatography (19:1 hexanes/EtOAc) afforded the product 2 in $87 \%$ yield (358 mg) as a colorless solid: mp $131-133{ }^{\circ} \mathrm{C} ;{ }^{1} \mathrm{H}$ NMR $\left(400 \mathrm{MHz}, \mathrm{CDCl}_{3}\right) \delta 8.58(\mathrm{~s}, 1 \mathrm{H}), 7.97$ $(\mathrm{dd}, J=13.9,8.4 \mathrm{~Hz}, 2 \mathrm{H}), 7.87(\mathrm{~d}, J=8.9 \mathrm{~Hz}, 2 \mathrm{H}), 7.65(\mathrm{t}, J=7.5 \mathrm{~Hz}, 1 \mathrm{H}), 7.57$ (t, $J=7.5 \mathrm{~Hz}$, $1 \mathrm{H}), 7.51(\mathrm{~d}, J=8.8 \mathrm{~Hz}, 2 \mathrm{H}), 7.38(\mathrm{~d}, J=8.8 \mathrm{~Hz}, 2 \mathrm{H}), 4.22(\mathrm{q}, J=11.2 \mathrm{~Hz}, 2 \mathrm{H}), 3.58(\mathrm{~s}, 1 \mathrm{H}) ;{ }^{13} \mathrm{C}$ NMR $\left(100 \mathrm{MHz}, \mathrm{CDCl}_{3}\right) \delta 190.2\left(\mathrm{dd}, J_{\mathrm{CF}}=29.9,27.8 \mathrm{~Hz}, 1 \mathrm{C}\right), 136.0,135.1,135.0,133.6(\mathrm{dd}$, $\left.J_{\mathrm{CF}}=5.7,4.1 \mathrm{~Hz}, 1 \mathrm{C}\right), 132.1,130.2(2 \mathrm{C}), 129.6,128.6(2 \mathrm{C}), 128.4(2 \mathrm{C}), 128.3,127.7,127.0,125.0$ $\left(\mathrm{t}, J_{\mathrm{CF}}=2.0 \mathrm{~Hz}, 1 \mathrm{C}\right), 116.0\left(\mathrm{dd}, J_{\mathrm{CF}}=266.3,264.2 \mathrm{~Hz}, 1 \mathrm{C}\right), 77.9\left(\mathrm{t}, J_{\mathrm{CF}}=24.8 \mathrm{~Hz}, 1 \mathrm{C}\right), 37.5(\mathrm{t}$, $\left.J_{\mathrm{CF}}=3.5 \mathrm{~Hz}, 1 \mathrm{C}\right) ;{ }^{19} \mathrm{~F} \mathrm{NMR}\left(376 \mathrm{MHz}, \mathrm{CDCl}_{3}\right) \delta-105.7\left(\mathrm{~d}, J_{F F}=274.5 \mathrm{~Hz}, 1 \mathrm{~F}\right),-107.2\left(\mathrm{~d}, J_{F F}=\right.$ $274.4 \mathrm{~Hz}, 1 \mathrm{~F}$ ); IR (film) $v_{\max } 3518,3061,2961,2925,1686,1626,1493 \mathrm{~cm}^{-1}$; HRMS (ESI-TOF) $m / z$ calcd for $\mathrm{C}_{20} \mathrm{H}_{14} \mathrm{BrClF}_{2} \mathrm{O}_{2} \mathrm{Na}(\mathrm{M}+\mathrm{Na})^{+} 460.9731$, found 460.9740 . 


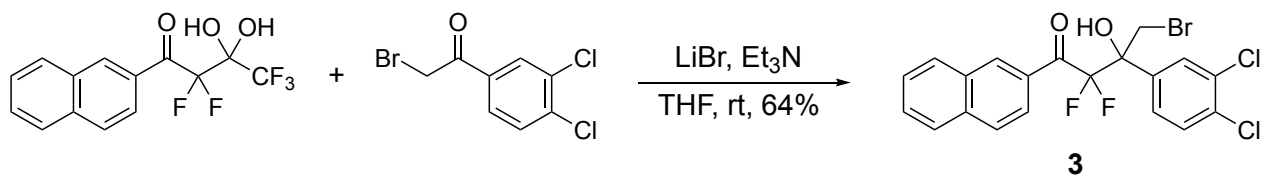

4-Bromo-3-(3,4-dichlorophenyl)-2,2-difluoro-3-hydroxy-1-(naphthalen-2-yl)butan-1-one 3 . See representative reaction procedure. A solution of 2,2,4,4,4-pentafluoro-3,3-dihydroxy-1(naphthalen-2-yl)-butan-1-one ${ }^{1}$ (100 mg, $\left.0.3 \mathrm{mmol}\right), 2$-bromo-3',4'-dichloroacetophenone (167 $\mathrm{mg}, 0.624 \mathrm{mmol})$, and $\mathrm{LiBr}(135 \mathrm{mg}, 1.56 \mathrm{mmol})$ in THF $(500 \mu \mathrm{L})$ was treated with $\mathrm{Et}_{3} \mathrm{~N}(53 \mu \mathrm{L}$, $0.37 \mathrm{mmol}) . \mathrm{SiO}_{2}$ flash chromatography (19:1 hexanes/EtOAc) afforded the product 3 in $64 \%$ yield (94 mg) as a pale yellow solid: $\mathrm{mp} 132-134{ }^{\circ} \mathrm{C} ;{ }^{1} \mathrm{H} \mathrm{NMR}\left(400 \mathrm{MHz}, \mathrm{CDCl}_{3}\right) \delta 8.60(\mathrm{~s}, 1 \mathrm{H})$, $7.98(\mathrm{dd}, J=11.2,8.3 \mathrm{~Hz}, 2 \mathrm{H}), 7.88(\mathrm{~d}, J=9.3 \mathrm{~Hz}, 2 \mathrm{H}), 7.70(\mathrm{~d}, J=2.2 \mathrm{~Hz}, 1 \mathrm{H}), 7.66(\mathrm{t}, J=7.5$ $\mathrm{Hz}, 1 \mathrm{H}), 7.58(\mathrm{t}, J=7.6 \mathrm{~Hz}, 1 \mathrm{H}), 7.48(\mathrm{~d}, J=8.5 \mathrm{~Hz}, 1 \mathrm{H}), 7.40(\mathrm{dd}, J=8.5,1.1 \mathrm{~Hz}, 1 \mathrm{H}), 4.23$ (d, $J=11.3 \mathrm{~Hz}, 1 \mathrm{H}), 4.13(\mathrm{~d}, J=11.3 \mathrm{~Hz}, 1 \mathrm{H}), 3.64(\mathrm{~s}, 1 \mathrm{H}) ;{ }^{13} \mathrm{C}$ NMR $\left(100 \mathrm{MHz}, \mathrm{CDCl}_{3}\right) \delta 190.0$ $\left(\mathrm{dd}, J_{\mathrm{CF}}=29.9,27.7 \mathrm{~Hz}, 1 \mathrm{C}\right), 136.9,136.1,133.6\left(\mathrm{dd}, J_{\mathrm{CF}}=5.8,4.0 \mathrm{~Hz}, 1 \mathrm{C}\right), 133.4,132.9,132.1$, $130.3,130.2,130.1,129.7,129.3,128.4,127.7,127.1,126.2,124.9\left(\mathrm{t}, J_{\mathrm{CF}}=2.1 \mathrm{~Hz}, 1 \mathrm{C}\right), 115.8$ $\left(\mathrm{dd}, J_{\mathrm{CF}}=265.6,262.4 \mathrm{~Hz}, 1 \mathrm{C}\right), 77.7\left(\mathrm{t}, J_{\mathrm{CF}}=25.0 \mathrm{~Hz}, 1 \mathrm{C}\right), 37.1\left(\mathrm{t}, J_{\mathrm{CF}}=3.3 \mathrm{~Hz}, 1 \mathrm{C}\right) ;{ }^{19} \mathrm{~F} \mathrm{NMR}$ $\left(376 \mathrm{MHz}, \mathrm{CDCl}_{3}\right) \delta-105.3\left(\mathrm{~d}, J_{F F}=277.6 \mathrm{~Hz}, 1 \mathrm{~F}\right),-106.9\left(\mathrm{~d}, J_{F F}=277.7 \mathrm{~Hz}, 1 \mathrm{~F}\right)$; IR (film) $v_{\max }$ $3519,3061,2924,1685,1626,1471 \mathrm{~cm}^{-1}$; HRMS (ESI-TOF) $\mathrm{m} / z$ calcd for $\mathrm{C}_{20} \mathrm{H}_{14} \mathrm{BrCl}_{2} \mathrm{~F}_{2} \mathrm{O}_{2}$ $(\mathrm{M}+\mathrm{H})^{+}$472.9522, found 472.9539 .

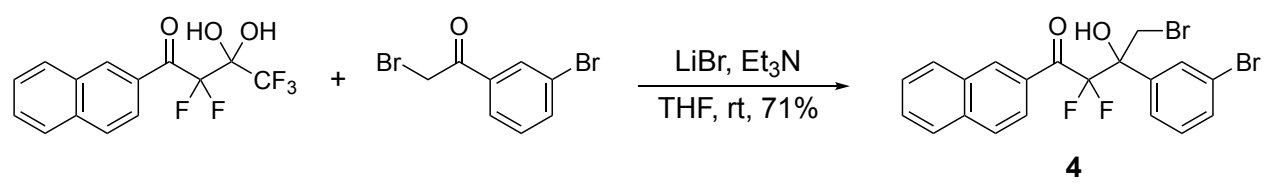

4-Bromo-3-(3-bromophenyl)-2,2-difluoro-3-hydroxy-1-(naphthalen-2-yl)butan-1-one 4. See representative reaction procedure. A solution of 2,2,4,4,4-pentafluoro-3,3-dihydroxy-1(naphthalen-2-yl)-butan-1-one ${ }^{1}(100 \mathrm{mg}, 0.3 \mathrm{mmol}), 2,3^{\prime}$-dibromoacetophenone (260 mg, 0.94 $\mathrm{mmol})$, and $\mathrm{LiBr}(135 \mathrm{mg}, 1.56 \mathrm{mmol})$ in THF $(500 \mu \mathrm{L})$ was treated with $\mathrm{Et}_{3} \mathrm{~N}(53 \mu \mathrm{L}, 0.37 \mathrm{mmol})$. $\mathrm{SiO}_{2}$ flash chromatography (19:1 hexanes/EtOAc) afforded the product 4 in $71 \%$ yield $(128 \mathrm{mg})$ as a colorless oil: ${ }^{1} \mathrm{H}$ NMR $\left(400 \mathrm{MHz}, \mathrm{CDCl}_{3}\right) \delta 8.58(\mathrm{~s}, 1 \mathrm{H}), 7.97(\mathrm{dd}, J=11.8,8.4 \mathrm{~Hz}, 2 \mathrm{H}), 7.87$ $(\mathrm{d}, J=8.5 \mathrm{~Hz}, 2 \mathrm{H}), 7.77(\mathrm{~s}, 1 \mathrm{H}), 7.66(\mathrm{ddd}, J=8.2,6.9,1.3 \mathrm{~Hz}, 1 \mathrm{H}), 7.57(\mathrm{ddd}, J=8.1,6.8,1.3$ $\mathrm{Hz}, 1 \mathrm{H}), 7.50(\mathrm{dd}, J=7.8,2.0 \mathrm{~Hz}, 2 \mathrm{H}), 7.29(\mathrm{t}, J=7.9 \mathrm{~Hz}, 1 \mathrm{H}), 4.24(\mathrm{~d}, J=10.6 \mathrm{~Hz}, 1 \mathrm{H}), 4.18$ $(\mathrm{d}, J=11.2 \mathrm{~Hz}, 1 \mathrm{H}), 3.62(\mathrm{~s}, 1 \mathrm{H}) ;{ }^{13} \mathrm{C} \mathrm{NMR}\left(100 \mathrm{MHz}, \mathrm{CDCl}_{3}\right) \delta 190.3\left(\mathrm{dd}, J_{\mathrm{CF}}=30.2,27.5 \mathrm{~Hz}\right.$, 1C), 139.0, 136.1, 133.7 (dd, $\left.J_{\mathrm{CF}}=6.1,3.7 \mathrm{~Hz}, 1 \mathrm{C}\right), 132.2(2 \mathrm{C}), 132.2,130.4,130.3,130.0,129.7$, $128.4,127.9,127.2,125.6,125.1,122.9,116.1\left(\mathrm{dd}, J_{\mathrm{CF}}=267.2,263.9 \mathrm{~Hz}, 1 \mathrm{C}\right), 78.0\left(\mathrm{t}, J_{\mathrm{CF}}=25.0\right.$ $\mathrm{Hz}, 1 \mathrm{C}), 37.6\left(\mathrm{t}, J_{\mathrm{CF}}=3.6 \mathrm{~Hz}, 1 \mathrm{C}\right) ;{ }^{19} \mathrm{~F} \mathrm{NMR}\left(376 \mathrm{MHz}, \mathrm{CDCl}_{3}\right) \delta-105.6\left(\mathrm{~d}, J_{F F}=274.7 \mathrm{~Hz}, 1 \mathrm{~F}\right)$, $-107.0\left(\mathrm{~d}, J_{F F}=274.7 \mathrm{~Hz}, 1 \mathrm{~F}\right)$; IR (film) $v_{\max } 3512,3062,2925,1682,1625 \mathrm{~cm}^{-1}$; HRMS (ESITOF) $m / z$ calcd for $\mathrm{C}_{20} \mathrm{H}_{15} \mathrm{Br}_{2} \mathrm{~F}_{2} \mathrm{O}_{2}(\mathrm{M}+\mathrm{H})^{+} 482.9407$, found 482.9433 .
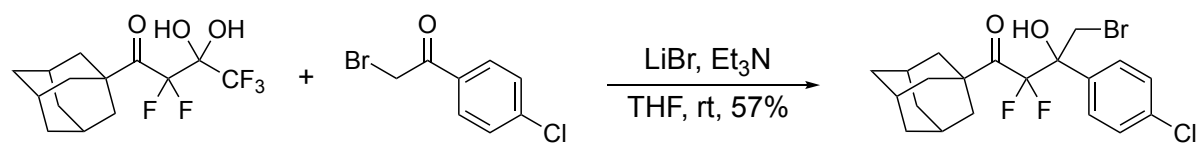

5

1-Adamantanyl-4-bromo-3-(4-chlorophenyl)-2,2-difluoro-3-hydroxybutan-1-one $5 . \quad$ See representative reaction procedure. A solution of 1-adamantanyl-2,2,4,4,4-pentafluoro-3,3dihydroxybutan-1-one ${ }^{1}$ (300 mg, $0.9 \mathrm{mmol}$ ), 2-bromo-4'-chloroacetophenone (427 $\mathrm{mg}, 1.83$ $\mathrm{mmol})$, and $\mathrm{LiBr}(397 \mathrm{mg}, 4.57 \mathrm{mmol})$ in $\mathrm{THF}(2 \mathrm{~mL})$ was treated with $\mathrm{Et}_{3} \mathrm{~N}(153 \mu \mathrm{L}, 1.10 \mathrm{mmol})$. 
$\mathrm{SiO}_{2}$ flash chromatography (19:2:1 hexanes/dichloromethane/EtOAc) afforded the product $\mathbf{5}$ in $57 \%$ yield $(236 \mathrm{mg})$ as a colorless solid: $\mathrm{mp} 106-108{ }^{\circ} \mathrm{C} ;{ }^{1} \mathrm{H} \mathrm{NMR}\left(500 \mathrm{MHz}, \mathrm{CDCl}_{3}\right) \delta 7.42(\mathrm{~d}$, $J=8.5 \mathrm{~Hz}, 2 \mathrm{H}), 7.37(\mathrm{~d}, J=8.7 \mathrm{~Hz}, 2 \mathrm{H}), 4.12(\mathrm{~d}, J=11.1 \mathrm{~Hz}, 1 \mathrm{H}), 4.02(\mathrm{~s}, 1 \mathrm{H}), 4.01(\mathrm{~d}, J=13.0$ $\mathrm{Hz}, 1 \mathrm{H}), 2.00(\mathrm{~s}, 3 \mathrm{H}), 1.79(\mathrm{~m}, 6 \mathrm{H}), 1.71(\mathrm{~d}, J=12.4 \mathrm{~Hz}, 3 \mathrm{H}), 1.64(\mathrm{~d}, J=10.7 \mathrm{~Hz}, 3 \mathrm{H}) ;{ }^{13} \mathrm{C} \mathrm{NMR}$ $\left(100 \mathrm{MHz}, \mathrm{CDCl}_{3}\right) \delta 206.3\left(\mathrm{dd}, J_{C F}=30.0,25.3 \mathrm{~Hz}, 1 \mathrm{C}\right), 135.6,135.0,128.6(2 \mathrm{C}), 128.5(2 \mathrm{C})$, $115.4\left(\mathrm{dd}, J_{C F}=271.4,267.1 \mathrm{~Hz}, 1 \mathrm{C}\right), 78.4\left(\mathrm{t}, J_{C F}=23.9 \mathrm{~Hz}\right), 47.6\left(\mathrm{~d}, J_{C F}=2.1 \mathrm{~Hz}\right), 37.1\left(\mathrm{t}, J_{C F}\right.$ $=3.8 \mathrm{~Hz}, 1 \mathrm{C}), 36.8\left(\mathrm{~d}, J_{C F}=2.3 \mathrm{~Hz}, 3 \mathrm{C}\right), 36.3(3 \mathrm{C}), 27.7(3 \mathrm{C}) ;{ }^{19} \mathrm{~F}$ NMR $\left(376 \mathrm{MHz}, \mathrm{CDCl}_{3}\right) \delta-$ $106.3\left(\mathrm{~d}, J_{F F}=284.2 \mathrm{~Hz}, 1 \mathrm{~F}\right),-110.4\left(\mathrm{~d}, J_{F F}=284.2 \mathrm{~Hz}, 1 \mathrm{~F}\right)$; IR (film) $v_{\max } 3520,2912,2856$, 1714, $1495 \mathrm{~cm}^{-1}$; HRMS (ESI-TOF) $\mathrm{m} / z$ calcd for $\mathrm{C}_{20} \mathrm{H}_{22} \mathrm{BrCl}_{2} \mathrm{~F}_{2} \mathrm{O}_{2}(\mathrm{M}+\mathrm{Cl})^{-} 481.0148$, found 481.0116 .

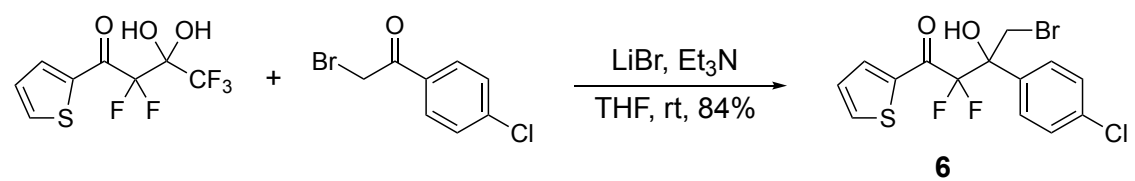

4-Bromo-3-(4-chlorophenyl)-2,2-difluoro-3-hydroxy-1-(thiophen-2-yl)butan-1-one 6. See representative reaction procedure. A solution of 2,2,4,4,4-pentafluoro-3,3-dihydroxy-1-(thiophen2-yl)butan-1-one ${ }^{2}$ (100 mg, $\left.0.36 \mathrm{mmol}\right), 2$-bromo-4'-chloroacetophenone (170 mg, $0.73 \mathrm{mmol}$ ), and $\mathrm{LiBr}(156 \mathrm{mg}, 1.82 \mathrm{mmol})$ in THF $(500 \mu \mathrm{L})$ was treated with $\mathrm{Et}_{3} \mathrm{~N}(61 \mu \mathrm{L}, 0.44 \mathrm{mmol}) . \mathrm{SiO}_{2}$ flash chromatography $(19: 1$ hexanes/EtOAc) afforded the product 6 in $84 \%$ yield $(121 \mathrm{mg})$ as colorless solid: mp 97-99 ${ }^{\circ} \mathrm{C} ;{ }^{1} \mathrm{H}$ NMR $\left(400 \mathrm{MHz}, \mathrm{CDCl}_{3}\right) \delta 7.91(\mathrm{~s}, 1 \mathrm{H}), 7.79(\mathrm{~d}, J=4.9 \mathrm{~Hz}, 1 \mathrm{H})$, $7.47(\mathrm{~d}, J=8.5 \mathrm{~Hz}, 2 \mathrm{H}), 7.36(\mathrm{~d}, J=8.8 \mathrm{~Hz}, 2 \mathrm{H}), 7.14(\mathrm{t}, J=4.5 \mathrm{~Hz}, 1 \mathrm{H}), 4.16(\mathrm{dd}, J=14.5,11.3$ $\mathrm{Hz}, 2 \mathrm{H}), 3.52(\mathrm{~s}, 1 \mathrm{H}) ;{ }^{13} \mathrm{C} \mathrm{NMR}\left(100 \mathrm{MHz}, \mathrm{CDCl}_{3}\right) \delta 182.5\left(\mathrm{dd}, J_{\mathrm{CF}}=30.9,28.9 \mathrm{~Hz}, 1 \mathrm{C}\right), 139.1$ $\left(\mathrm{d}, J_{\mathrm{CF}}=0.8 \mathrm{~Hz}, 1 \mathrm{C}\right), 137.3,137.0\left(\mathrm{dd}, J_{\mathrm{CF}}=7.0,4.5 \mathrm{~Hz}, 1 \mathrm{C}\right), 135.1,134.8,128.8,128.6(2 \mathrm{C})$, $128.4(2 \mathrm{C}), 115.5\left(\mathrm{q}, J_{\mathrm{CF}}=266.5,263.6 \mathrm{~Hz}, 1 \mathrm{C}\right), 77.7\left(\mathrm{t}, J_{\mathrm{CF}}=24.9 \mathrm{~Hz}, 1 \mathrm{C}\right), 37.2\left(\mathrm{t}, J_{\mathrm{CF}}=3.2 \mathrm{~Hz}\right.$, $1 \mathrm{C}) ;{ }^{19} \mathrm{~F}$ NMR $\left(376 \mathrm{MHz}, \mathrm{CDCl}_{3}\right) \delta-108.6\left(\mathrm{~d}, J_{F F}=266.3 \mathrm{~Hz}, 1 \mathrm{~F}\right),-109.7\left(\mathrm{~d}, J_{F F}=266.6 \mathrm{~Hz}\right.$, 1F); IR (film) $v_{\max }$ 3501, 3108, 1659, $1409 \mathrm{~cm}^{-1}$; HRMS (ESI-TOF) $\mathrm{m} / z$ calcd for $\mathrm{C}_{14} \mathrm{H}_{10} \mathrm{BrCl}_{2} \mathrm{~F}_{2} \mathrm{O}_{2} \mathrm{~S}(\mathrm{M}+\mathrm{Cl})^{-} 428.8930$, found 428.8904 .
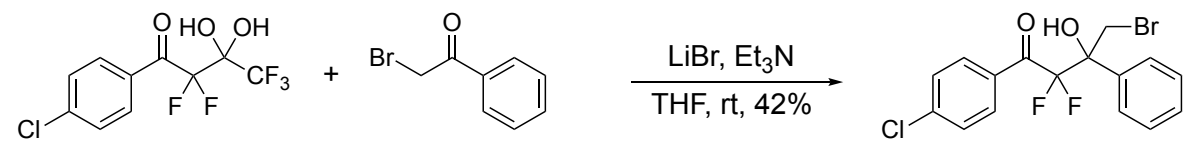

7

4-Bromo-1-(4-chlorophenyl)-2,2-difluoro-3-hydroxy-3-phenylbutan-1-one $7 . \quad$ See representative reaction procedure. A solution of 1-(4-chlorophenyl)-2,2,4,4,4-pentafluoro-3,3dihydroxybutan-1-one ${ }^{1}(120 \mathrm{mg}, 0.37 \mathrm{mmol})$, 2-chloroacetophenone (376 $\left.\mathrm{mg}, 1.88 \mathrm{mmol}\right)$, and $\mathrm{LiBr}(163 \mathrm{mg}, 1.88 \mathrm{mmol})$ in THF $(1.5 \mathrm{~mL})$ was treated with $\mathrm{Et}_{3} \mathrm{~N}(53 \mu \mathrm{L}, 0.45 \mathrm{mmol}) . \mathrm{SiO}_{2}$ flash chromatography (hexanes $\rightarrow 50: 1$ hexanes/EtOAc) afforded the product 7 in $42 \%$ yield (64 $\mathrm{mg}$ ) as a pale yellow oil: ${ }^{1} \mathrm{H} \mathrm{NMR}\left(500 \mathrm{MHz}, \mathrm{CDCl}_{3}\right) \delta 7.93(\mathrm{~d}, J=8.3 \mathrm{~Hz}, 2 \mathrm{H}), 7.52(\mathrm{~d}, J=7.0 \mathrm{~Hz}$, 2H), 7.44-7.38 (m, 5H), $7.22(\mathrm{~d}, J=11.2 \mathrm{~Hz}, 1 \mathrm{H}), 4.19(\mathrm{~d}, J=11.1 \mathrm{~Hz}, 1 \mathrm{H}), 3.37$ (br s, $1 \mathrm{H}) ;{ }^{13} \mathrm{C}$ NMR $\left(125 \mathrm{MHz}, \mathrm{CDCl}_{3}\right) \delta 189.6(\mathrm{dd}, J=30.5,27.3 \mathrm{~Hz}, 1 \mathrm{C}), 141.2,136.23,132.13(\mathrm{dd}, J=4.4$, $2.9 \mathrm{~Hz}, 1 \mathrm{C}), 131.8(\mathrm{dd}, J=1.8 \mathrm{~Hz}, 1 \mathrm{C}), 129.2$ (2C), 128.9 (2C), $128.6(2 \mathrm{C}), 126.9$ (2C), 116.4 (dd, $J=265.7,263.1 \mathrm{~Hz}, 1 \mathrm{C}), 78.0(\mathrm{t}, J=25.1 \mathrm{~Hz}, 1 \mathrm{C}), 38.01(\mathrm{t}, J=3.5 \mathrm{~Hz}, 1 \mathrm{C}) ;{ }^{19} \mathrm{~F} \mathrm{NMR}(376 \mathrm{MHz}$, $\left.\mathrm{CDCl}_{3}\right) \delta-106.77\left(\mathrm{~d}, J_{F F}=267.4 \mathrm{~Hz}, 1 \mathrm{~F}\right),-108.26\left(\mathrm{~d}, J_{F F}=267.5 \mathrm{~Hz}, 1 \mathrm{~F}\right)$; IR (film) $v_{\max } 3477$, $1692,1587 \mathrm{~cm}^{-1}$; HRMS (ESI-TOF) $\mathrm{m} / z$ calcd for $\mathrm{C}_{16} \mathrm{H}_{11} \mathrm{BrClF}_{2} \mathrm{O}_{2}(\mathrm{M}-\mathrm{H})^{-}$386.9599, found 386.9604 . 


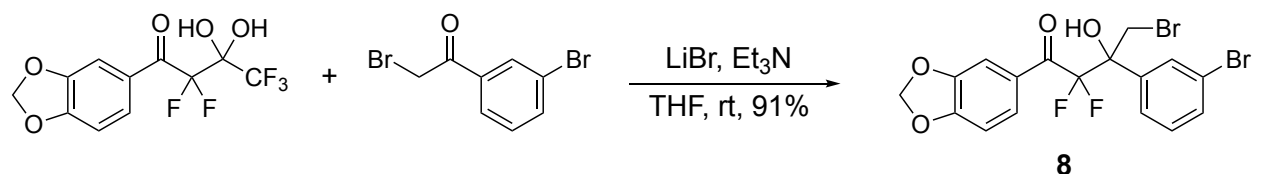

1-(Benzo[1,3]dioxol-5-yl)-4-bromo-3-(3-bromophenyl)-2,2-difluoro-3-hydroxybutan-1-one

8. See representative reaction procedure. A solution of 1-(benzo[1,3]dioxol-5-yl)-2,2,4,4,4pentafluoro-3,3-dihydroxybutan-1-one ${ }^{1}(100 \mathrm{mg}, 0.3 \mathrm{mmol}), 2,3^{\prime}$-dibromoacetophenone (177 mg, $0.637 \mathrm{mmol})$, and $\mathrm{LiBr}(138 \mathrm{mg}, 1.59 \mathrm{mmol})$ in THF $(500 \mu \mathrm{L})$ was treated with $\mathrm{Et}_{3} \mathrm{~N}(53 \mu \mathrm{L}, 0.38$ mmol). $\mathrm{SiO}_{2}$ flash chromatography (9:1 hexanes/EtOAc) afforded the product 8 in $91 \%$ yield (139 $\mathrm{mg})$ as a colorless oil: ${ }^{1} \mathrm{H} \mathrm{NMR}\left(400 \mathrm{MHz}, \mathrm{CDCl}_{3}\right) \delta 7.76-7.67(\mathrm{~m}, 2 \mathrm{H}), 7.51$ (ddd, $J=7.9,1.9$, $1.0 \mathrm{~Hz}, 1 \mathrm{H}), 7.49-7.44(\mathrm{~m}, 2 \mathrm{H}), 7.28(\mathrm{t}, J=7.9 \mathrm{~Hz}, 1 \mathrm{H}), 6.85(\mathrm{~d}, J=8.4 \mathrm{~Hz}, 1 \mathrm{H}), 6.07(\mathrm{~s}, 2 \mathrm{H})$, $4.16(\mathrm{~d}, J=11.2 \mathrm{~Hz}, 1 \mathrm{H}), 4.11(\mathrm{dd}, J=11.0,1.8 \mathrm{~Hz}, 1 \mathrm{H}), 3.62(\mathrm{~s}, 1 \mathrm{H}) ;{ }^{13} \mathrm{C} \mathrm{NMR}(100 \mathrm{MHz}$, $\left.\mathrm{CDCl}_{3}\right) \delta 188.2\left(\mathrm{dd}, J_{\mathrm{CF}}=30.3,27.6 \mathrm{~Hz}, 1 \mathrm{C}\right), 153.4,148.1,139.0,132.1,130.3,129.9,128.3(\mathrm{dd}$, $\left.J_{\mathrm{CF}}=5.7,3.6 \mathrm{~Hz}, 1 \mathrm{C}\right), 127.4,125.6,122.8,115.9\left(\mathrm{dd}, J_{\mathrm{CF}}=267.5,263.5 \mathrm{~Hz}, 1 \mathrm{C}\right), 110.2\left(\mathrm{dd}, J_{\mathrm{CF}}\right.$ $=4.3,2.5 \mathrm{~Hz}, 1 \mathrm{C}), 108.2,102.3,77.9\left(\mathrm{t}, J_{\mathrm{CF}}=25.5,24.4 \mathrm{~Hz}, 1 \mathrm{C}\right), 37.5\left(\mathrm{t}, J_{\mathrm{CF}}=3.5 \mathrm{~Hz}, 1 \mathrm{C}\right) ;{ }^{19} \mathrm{~F}$ $\operatorname{NMR}\left(376 \mathrm{MHz}, \mathrm{CDCl}_{3}\right) \delta-105.2\left(\mathrm{~d}, J_{F F}=275.8 \mathrm{~Hz}, 1 \mathrm{~F}\right),-106.7\left(\mathrm{~d}, J_{F F}=275.9 \mathrm{~Hz}, 1 \mathrm{~F}\right)$; IR (film) $v_{\max }$ 3507, 3079, 2910, 1680, 1605, 1445, $1268 \mathrm{~cm}^{-1}$; HRMS (ESI-TOF) $\mathrm{m} / z$ calcd for $\mathrm{C}_{17} \mathrm{H}_{12} \mathrm{ClBr}_{2} \mathrm{~F}_{2} \mathrm{O}_{4}(\mathrm{M}+\mathrm{Cl})^{-} 510.8759$, found 510.8751 .

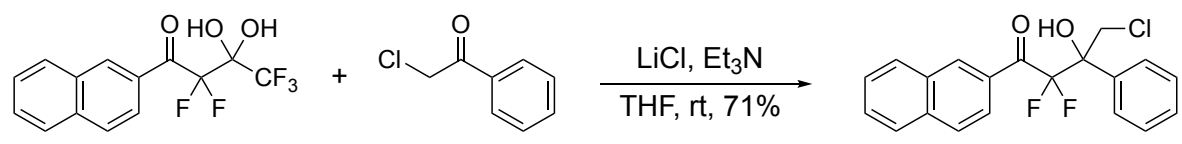

9

4-Chloro-2,2-difluoro-3-hydroxy-1-(naphthalen-2-yl)-3-phenylbutan-1-one $9 . \quad$ See representative reaction procedure. A solution of 2,2,4,4,4-pentafluoro-3,3-dihydroxy-1(naphthalen-2-yl)-butan-1-one ${ }^{1}$ (100 mg, $\left.0.3 \mathrm{mmol}\right), 2$-chloroacetophenone (97 mg, $\left.0.62 \mathrm{mmol}\right)$, and $\mathrm{LiCl}(66 \mathrm{mg}, 1.6 \mathrm{mmol})$ in THF $(500 \mu \mathrm{L})$ was treated with $\mathrm{Et}_{3} \mathrm{~N}\left(53 \mu \mathrm{L}, 0.37 \mathrm{mmol}^{2} . \mathrm{SiO}_{2}\right.$ flash chromatography (19:1 hexanes/EtOAc) afforded the product 9 in $71 \%$ yield $(80 \mathrm{mg})$ as a colorless film: ${ }^{1} \mathrm{H}$ NMR $\left(400 \mathrm{MHz}, \mathrm{CDCl}_{3}\right) \delta 8.53(\mathrm{~s}, 1 \mathrm{H}), 7.95(\mathrm{dd}, J=14.3,8.2 \mathrm{~Hz}, 2 \mathrm{H}), 7.85$ $(\mathrm{dd}, J=8.4,4.5 \mathrm{~Hz}, 2 \mathrm{H}), 7.67-7.53(\mathrm{~m}, 4 \mathrm{H}), 7.44-7.36(\mathrm{~m}, 3 \mathrm{H}), 4.37(\mathrm{~s}, 2 \mathrm{H}), 3.61(\mathrm{~s}, 1 \mathrm{H}) ;{ }^{13} \mathrm{C}$ $\operatorname{NMR}\left(100 \mathrm{MHz}, \mathrm{CDCl}_{3}\right) \delta 190.6\left(\mathrm{dd}, J_{\mathrm{CF}}=30.0,27.8 \mathrm{~Hz}, 1 \mathrm{C}\right), 136.1,136.0,133.7\left(\mathrm{dd}, J_{\mathrm{CF}}=5.5\right.$, 4.1 Hz, 1C), 132.3, 130.7, 130.4, 129.6, 129.1, 128.6 (2C), 128.3, 127.8, 127.1 (2C), 127.1, 125.2 $\left(\mathrm{t}, J_{\mathrm{CF}}=2.2 \mathrm{~Hz}, 1 \mathrm{C}\right), 116.9\left(\mathrm{dd}, J_{\mathrm{CF}}=264.1,262.0 \mathrm{~Hz}, 1 \mathrm{C}\right), 78.6\left(\mathrm{t}, J_{\mathrm{CF}}=24.8 \mathrm{~Hz}, 1 \mathrm{C}\right), 48.3(\mathrm{t}$, $\left.J_{\mathrm{CF}}=3.9 \mathrm{~Hz}, 1 \mathrm{C}\right) ;{ }^{19} \mathrm{~F}$ NMR $\left(376 \mathrm{MHz}, \mathrm{CDCl}_{3}\right) \delta-106.6\left(\mathrm{~d}, J_{F F}=271.8 \mathrm{~Hz}, 1 \mathrm{~F}\right),-107.8\left(\mathrm{~d}, J_{F F}\right.$ $=271.8 \mathrm{~Hz}, 1 \mathrm{~F}$ ); IR (film) $v_{\max } 3529,3061,2925,1685,1625,1450 \mathrm{~cm}^{-1}$; HRMS (ESI-TOF) $\mathrm{m} / z$ calcd for $\mathrm{C}_{20} \mathrm{H}_{16} \mathrm{ClF}_{2} \mathrm{O}_{2}(\mathrm{M}+\mathrm{H})^{+} 361.0807$, found 361.0782 .

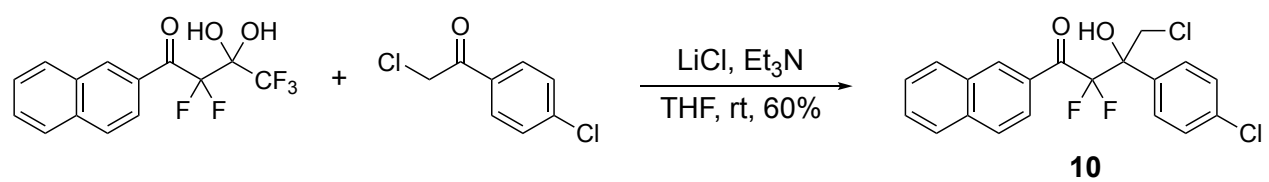

4-Chloro-3-(4-chlorophenyl)-2,2-difluoro-3-hydroxy-1-(naphthalen-2-yl)butan-1-one 10. See representative reaction procedure. A solution of 2,2,4,4,4-pentafluoro-3,3-dihydroxy-1(naphthalen-2-yl)-butan-1-one ${ }^{1}(30 \mathrm{mg}, 0.09 \mathrm{mmol}), 2,4^{\prime}$-dichloroacetophenone (35 mg, 0.19 $\mathrm{mmol})$, and $\mathrm{LiCl}(26 \mathrm{mg}, 0.61 \mathrm{mmol})$ in THF $(500 \mu \mathrm{L})$ was treated with $\mathrm{Et}_{3} \mathrm{~N}(26 \mu \mathrm{L}, 0.19 \mathrm{mmol})$. $\mathrm{SiO}_{2}$ flash chromatography (19:1 hexanes/EtOAc) afforded the product $\mathbf{1 0}$ in $60 \%$ yield $(22 \mathrm{mg})$ as a colorless solid: mp $107-109{ }^{\circ} \mathrm{C} ;{ }^{1} \mathrm{H}$ NMR $\left(400 \mathrm{MHz}, \mathrm{CDCl}_{3}\right) \delta 8.59$ (s, 1H), $7.99(\mathrm{~d}, J=8.7$ 
$\mathrm{Hz}, 1 \mathrm{H}), 7.95(\mathrm{~d}, J=8.2 \mathrm{~Hz}, 1 \mathrm{H}), 7.86(\mathrm{~m}, 2 \mathrm{H}) .7 .65(\mathrm{td}, J=7.0,1.1 \mathrm{~Hz}, 1 \mathrm{H}), 7.57(\mathrm{td}, J=8.0$, $1.0 \mathrm{~Hz}, 1 \mathrm{H}), 7.54(\mathrm{~d}, J=8.8 \mathrm{~Hz}, 2 \mathrm{H}), 7.39(\mathrm{~d}, J=8.7 \mathrm{~Hz}, 2 \mathrm{H}), 4.37(\mathrm{~d}, J=11.9 \mathrm{~Hz}, 1 \mathrm{H}), 4.32(\mathrm{~d}$, $J=11.9 \mathrm{~Hz}, 1 \mathrm{H}), 3.73($ br s, $1 \mathrm{H}) ;{ }^{13} \mathrm{C} \mathrm{NMR}\left(100 \mathrm{MHz}, \mathrm{CDCl}_{3}\right) \delta 190.2\left(\mathrm{t}, J_{\mathrm{CF}}=27.9 \mathrm{~Hz}, 1 \mathrm{C}\right)$, $136.0,135.1,134.5,133.6\left(\mathrm{dd}, J_{\mathrm{CF}}=4.2,4.2 \mathrm{~Hz}, 1 \mathrm{C}\right), 132.1,130.3,130.2,129.6,128.6(2 \mathrm{C})$, $128.5(2 \mathrm{C}), 128.3127 .7,127.0,124.9\left(\mathrm{t}, J_{\mathrm{CF}}=2.1 \mathrm{~Hz}, 1 \mathrm{C}\right), 116.5\left(\mathrm{dd}, J_{\mathrm{CF}}=267.5,263.2 \mathrm{~Hz}, 1 \mathrm{C}\right)$, $78.3\left(\mathrm{t}, J_{\mathrm{CF}}=24.8 \mathrm{~Hz}, 1 \mathrm{C}\right), 47.9\left(\mathrm{t}, J_{\mathrm{CF}}=3.8 \mathrm{~Hz}, 1 \mathrm{C}\right) ;{ }^{19} \mathrm{~F} \mathrm{NMR}\left(376 \mathrm{MHz}, \mathrm{CDCl}_{3}\right) \delta-106.3(\mathrm{~d}$, $\left.J_{F F}=275.8 \mathrm{~Hz}, 1 \mathrm{~F}\right),-107.7\left(\mathrm{~d}, J_{F F}=275.6 \mathrm{~Hz}, 1 \mathrm{~F}\right)$; IR (film) $v_{\max } 3513,3060,2925,1682,1671$, $1624,1495 \mathrm{~cm}^{-1}$; HRMS (ESI-TOF) $\mathrm{m} / z$ calcd for $\mathrm{C}_{20} \mathrm{H}_{14} \mathrm{Cl}_{2} \mathrm{~F}_{2} \mathrm{NaO}_{2}(\mathrm{M}+\mathrm{Na})^{+} 417.0237$, found 417.0242 .

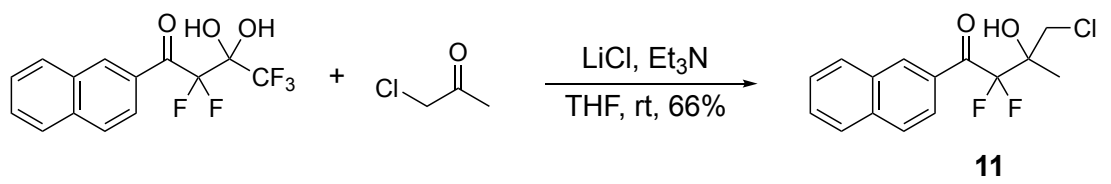

4-Chloro-2,2-difluoro-3-hydroxy-3-methyl-1-(naphthalen-2-yl)butan-1-one $11 . \quad$ See representative reaction procedure. A solution of 2,2,4,4,4-pentafluoro-3,3-dihydroxy-1(naphthalen-2-yl)-butan-1-one ${ }^{1}(100 \mathrm{mg}, 0.3 \mathrm{mmol})$, chloroacetone $(125 \mu \mathrm{L}, 1.56 \mathrm{mmol})$, and $\mathrm{LiCl}$ (66 mg, $1.6 \mathrm{mmol})$ in THF $(500 \mu \mathrm{L})$ was treated with $\mathrm{Et}_{3} \mathrm{~N}(53 \mu \mathrm{L}, 0.37 \mathrm{mmol}) . \mathrm{SiO}_{2}$ flash chromatography (19:1 hexanes/EtOAc) afforded the product 11 in $66 \%$ yield (68 $\mathrm{mg})$ as a colorless oil: ${ }^{1} \mathrm{H}$ NMR $\left(400 \mathrm{MHz}, \mathrm{CDCl}_{3}\right) \delta 8.73(\mathrm{~s}, 1 \mathrm{H}), 8.09(\mathrm{~d}, J=8.3 \mathrm{~Hz}, 1 \mathrm{H}), 8.00(\mathrm{~d}, J=8.1 \mathrm{~Hz}, 1 \mathrm{H})$, $7.93-7.87(\mathrm{~m}, 2 \mathrm{H}), 7.66(\mathrm{t}, J=8.1 \mathrm{~Hz}, 1 \mathrm{H}), 7.58(\mathrm{t}, J=8.0 \mathrm{~Hz}, 1 \mathrm{H}), 4.01(\mathrm{~d}, J=11.6 \mathrm{~Hz}, 1 \mathrm{H})$, $3.80(\mathrm{~d}, J=11.6 \mathrm{~Hz}, 1 \mathrm{H}), 3.09(\mathrm{~s}, 1 \mathrm{H}), 1.60(\mathrm{~s}, 3 \mathrm{H}) ;{ }^{13} \mathrm{C} \mathrm{NMR}\left(100 \mathrm{MHz}, \mathrm{CDCl}_{3}\right) \delta 190.6\left(\mathrm{t}, J_{\mathrm{CF}}\right.$ $=29.8 \mathrm{~Hz}, 1 \mathrm{C}), 136.1,133.8\left(\mathrm{t}, J_{\mathrm{CF}}=4.9 \mathrm{~Hz}, 1 \mathrm{C}\right), 132.3,130.3,130.2,129.6,128.4,127.8,127.1$, $125.1,117.2\left(\mathrm{t}, J_{\mathrm{CF}}=263.3 \mathrm{~Hz}, 1 \mathrm{C}\right), 75.2\left(\mathrm{t}, J_{\mathrm{CF}}=24.4 \mathrm{~Hz}, 1 \mathrm{C}\right), 48.6\left(\mathrm{t}, J_{\mathrm{CF}}=3.9 \mathrm{~Hz}, 1 \mathrm{C}\right), 19.7$ $\left(\mathrm{t}, J_{\mathrm{CF}}=3.9 \mathrm{~Hz}, 1 \mathrm{C}\right) ;{ }^{19} \mathrm{~F} \mathrm{NMR}\left(376 \mathrm{MHz}, \mathrm{CDCl}_{3}\right) \delta-108.9\left(\mathrm{~d}, J_{F F}=284.0 \mathrm{~Hz}, 1 \mathrm{~F}\right),-110.6\left(\mathrm{~d}, J_{F F}\right.$ $=284.0 \mathrm{~Hz} 1 \mathrm{~F}$ ); IR (film) $v_{\max } 3361,2924,2853,1686,1662,1625,1494 \mathrm{~cm}^{-1}$; HRMS (ESI-TOF) $m / z$ calcd for $\mathrm{C}_{15} \mathrm{H}_{14} \mathrm{ClF}_{2} \mathrm{O}_{2}(\mathrm{M}+\mathrm{H})^{+} 299.0650$, found 299.0623 .
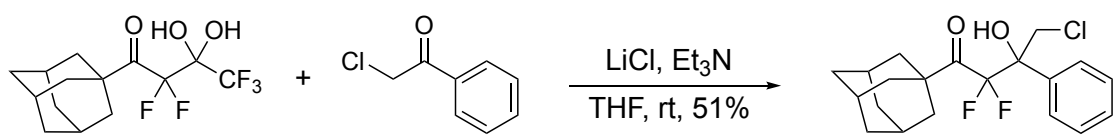

12

1-Adamantanyl-4-chloro-2,2-difluoro-3-hydroxy-3-phenylbutan-1-one 12. See representative reaction procedure. A solution of 1-adamantanyl)-2,2,4,4,4-pentafluoro-3,3-dihydroxybutan-1one $^{1}$ (100 mg, $\left.0.304 \mathrm{mmol}\right)$, 2-chloroacetophenone (94 mg, $\left.0.61 \mathrm{mmol}\right)$, and $\mathrm{LiCl}$ (65 mg, 1.5 mmol) in THF $(500 \mu \mathrm{L})$ was treated with $\mathrm{Et}_{3} \mathrm{~N}(51 \mu \mathrm{L}, 0.37 \mathrm{mmol}) . \mathrm{SiO}_{2}$ flash chromatography (19:1 hexanes/EtOAc) afforded the product 12 in $51 \%$ yield $(57 \mathrm{mg})$ as a colorless solid: $102-103$ $\mathrm{mp}{ }^{\circ} \mathrm{C} ;{ }^{1} \mathrm{H}$ NMR $\left(400 \mathrm{MHz}, \mathrm{CDCl}_{3}\right) \delta 7.50(\mathrm{~d}, J=7.5 \mathrm{~Hz}, 2 \mathrm{H}), 7.43-7.33(\mathrm{~m}, 3 \mathrm{H}), 4.28(\mathrm{~d}, J=$ $11.8 \mathrm{~Hz}, 1 \mathrm{H}), 4.21$ (d, $J=11.7 \mathrm{~Hz}, 1 \mathrm{H}), 4.08$ (br s, 1H), $1.98(\mathrm{~s}, 3 \mathrm{H}), 1.83-1.74(\mathrm{~m}, 6 \mathrm{H}), 1.73-$ $1.58(\mathrm{~m}, 6 \mathrm{H}) ;{ }^{13} \mathrm{C} \mathrm{NMR}\left(100 \mathrm{MHz}, \mathrm{CDCl}_{3}\right) \delta 206.5\left(\mathrm{dd}, J_{\mathrm{CF}}=29.2,24.3 \mathrm{~Hz}, 1 \mathrm{C}\right), 136.3,128.9$, $128.5(2 \mathrm{C}), 127.1(2 \mathrm{C}), 116.1\left(\mathrm{dd}, J_{\mathrm{CF}}=269.0,264.7 \mathrm{~Hz}, 1 \mathrm{C}\right), 79.0\left(\mathrm{t}, J_{\mathrm{CF}}=23.7 \mathrm{~Hz}, 1 \mathrm{C}\right), 48.0(\mathrm{t}$, $\left.J_{\mathrm{CF}}=4.1 \mathrm{~Hz}, 1 \mathrm{C}\right), 36.7\left(\mathrm{~d}, J_{\mathrm{CF}}=2.2 \mathrm{~Hz}, 3 \mathrm{C}\right), 36.4(3 \mathrm{C}), 27.7(3 \mathrm{C}) ;{ }^{19} \mathrm{~F}$ NMR $\left(376 \mathrm{MHz}, \mathrm{CDCl}_{3}\right)$ $\delta-106.7\left(\mathrm{~d}, J_{F F}=282.3 \mathrm{~Hz}, 1 \mathrm{~F}\right),-111.2\left(\mathrm{~d}, J_{F F}=282.0 \mathrm{~Hz}, 1 \mathrm{~F}\right)$; IR (film) $v_{\max } 3506,2912,2856$, 1716, $1452 \mathrm{~cm}^{-1}$; HRMS (ESI-TOF) $\mathrm{m} / z$ calcd for $\mathrm{C}_{20} \mathrm{H}_{23} \mathrm{ClF}_{2} \mathrm{NaO}_{2}(\mathrm{M}+\mathrm{Na})^{+} 391.1252$, found 391.1230 . 

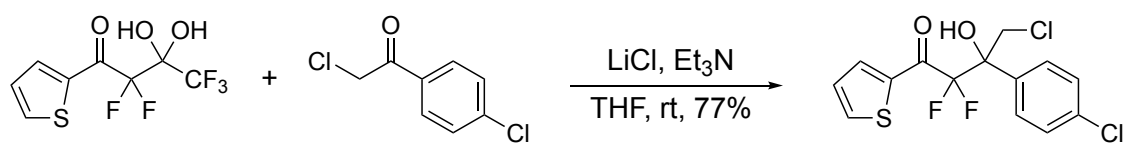

13

4-Chloro-3-(4-chlorophenyl)-2,2-difluoro-3-hydroxy-1-(thiophen-2-yl)butan-1-one 13. See representative reaction procedure. A solution of 2,2,4,4,4-pentafluoro-3,3-dihydroxy-1-(thiophen2-yl)butan-1-one ${ }^{2}$ (55 mg, $0.20 \mathrm{mmol}$ ), 2,4'-dichloroacetophenone (76 mg, $0.04 \mathrm{mmol}$ ), and $\mathrm{LiCl}$ $(42 \mathrm{mg}, 1.0 \mathrm{mmol})$ in THF $(500 \mu \mathrm{L})$ was treated with $\mathrm{Et}_{3} \mathrm{~N}(33 \mu \mathrm{L}, 0.24 \mathrm{mmol}) . \mathrm{SiO}_{2}$ flash chromatography (19:1 hexanes/EtOAc) afforded the product 13 in 77\% yield (54 $\mathrm{mg})$ as a colorless oil: ${ }^{1} \mathrm{H}$ NMR $\left(400 \mathrm{MHz}, \mathrm{CDCl}_{3}\right) \delta 7.92(\mathrm{~m}, 1 \mathrm{H}), 7.79(\mathrm{dd}, J=4.9,1.0 \mathrm{~Hz}, 1 \mathrm{H}), 7.49(\mathrm{~d}, J=8.5$ $\mathrm{Hz}, 2 \mathrm{H}), 7.37(\mathrm{~d}, J=8.8 \mathrm{~Hz}, 2 \mathrm{H}), 7.15(\mathrm{dd}, J=4.8,4.1 \mathrm{~Hz}, 1 \mathrm{H}), 4.30(\mathrm{~d}, J=11.2 \mathrm{~Hz}, 1 \mathrm{H}), 4.26$ $(\mathrm{d}, J=12.4 \mathrm{~Hz}, 1 \mathrm{H}), 3.55(\mathrm{~s}, 1 \mathrm{H}) ;{ }^{13} \mathrm{C}$ DEPTQ NMR $\left(100 \mathrm{MHz}, \mathrm{CDCl}_{3}\right) \delta 182.7\left(\mathrm{t}, J_{\mathrm{CF}}=29.9 \mathrm{~Hz}\right.$, 1C), 139.1, 137.4, 137.1 (dd, $\left.J_{\mathrm{CF}}=6.6,5.2 \mathrm{~Hz}, 1 \mathrm{C}\right), 135.3,134.4,128.9,128.8$ (2C), $128.6(2 \mathrm{C})$, $116.1\left(\mathrm{t}, J_{\mathrm{CF}}=267.5 \mathrm{~Hz}, 1 \mathrm{C}\right), 78.3\left(\mathrm{t}, J_{\mathrm{CF}}=24.7 \mathrm{~Hz}, 1 \mathrm{C}\right), 47.9\left(\mathrm{t}, J_{\mathrm{CF}}=3.6 \mathrm{~Hz}, 1 \mathrm{C}\right) ;{ }^{19} \mathrm{~F} \mathrm{NMR}(376$ $\left.\mathrm{MHz}, \mathrm{CDCl}_{3}\right) \delta-109.0\left(\mathrm{~d}, J_{F F}=268.6 \mathrm{~Hz}, 1 \mathrm{~F}\right),-110.0\left(\mathrm{~d}, J_{F F}=268.6 \mathrm{~Hz}, 1 \mathrm{~F}\right)$; IR (film) $v_{\max }$ 3493, 3115, 2926, 1666, $1414 \mathrm{~cm}^{-1}$; HRMS (ESI-TOF) $\mathrm{m} / z$ calcd for $\mathrm{C}_{14} \mathrm{H}_{11} \mathrm{Cl}_{2} \mathrm{~F}_{2} \mathrm{O}_{2} \mathrm{~S}(\mathrm{M}+\mathrm{H})^{+}$ 350.9825 , found 350.9849 .

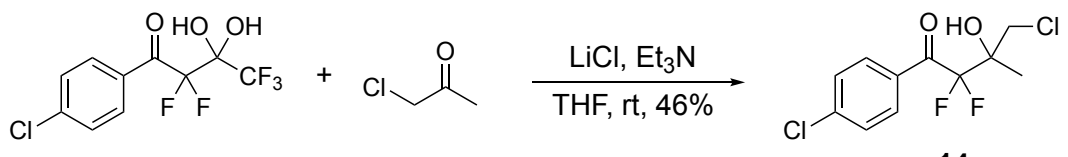

14

4-Chloro-1-(4-chlorophenyl)-2,2-difluoro-3-hydroxy-3-methylbutan-1-one $14 . \quad$ See representative reaction procedure. A solution of 1-(4-chlorophenyl)-2,2,4,4,4-pentafluoro-3,3dihydroxybutan-1-one ${ }^{1}(65 \mathrm{mg}, 0.21 \mathrm{mmol})$, chloroacetone $(85 \mu \mathrm{L}, 1.1 \mathrm{mmol})$, and $\mathrm{LiCl}(45 \mathrm{mg}$, $1.1 \mathrm{mmol})$ in THF $(500 \mu \mathrm{L})$ was treated with $\mathrm{Et}_{3} \mathrm{~N}(36 \mu \mathrm{L}, 0.26 \mathrm{mmol}) . \mathrm{SiO}_{2}$ flash chromatography (19:1 hexanes/EtOAc) afforded the product 14 in $46 \%$ yield $(30 \mathrm{mg})$ as a colorless oil: ${ }^{1} \mathrm{H}$ NMR $\left(400 \mathrm{MHz}, \mathrm{CDCl}_{3}\right) \delta 8.06(\mathrm{~d}, J=8.6 \mathrm{~Hz}, 2 \mathrm{H}), 7.47(\mathrm{~d}, J=8.7 \mathrm{~Hz}, 2 \mathrm{H}), 3.95(\mathrm{~d}, J=11.7 \mathrm{~Hz}, 1 \mathrm{H})$, $3.75(\mathrm{~d}, J=11.7 \mathrm{~Hz}, 1 \mathrm{H}), 2.92(\mathrm{~s}, 1 \mathrm{H}), 1.55(\mathrm{~s}, 3 \mathrm{H}) ;{ }^{13} \mathrm{C}$ NMR $\left(100 \mathrm{MHz}, \mathrm{CDCl}_{3}\right) \delta 189.7\left(\mathrm{t}, J_{\mathrm{CF}}\right.$ $=30.3 \mathrm{~Hz}, 1 \mathrm{C}), 141.5,132.2\left(\mathrm{t}, J_{\mathrm{CF}}=3.7 \mathrm{~Hz}, 2 \mathrm{C}\right), 131.5\left(\mathrm{t}, J_{\mathrm{CF}}=2.0 \mathrm{~Hz}, 1 \mathrm{C}\right), 129.1(2 \mathrm{C}), 117.1$ $\left(\mathrm{t}, J_{\mathrm{CF}}=261.0 \mathrm{~Hz}, 1 \mathrm{C}\right), 75.1\left(\mathrm{t}, J_{\mathrm{CF}}=24.7 \mathrm{~Hz}, 1 \mathrm{C}\right), 48.6\left(\mathrm{dd}, J_{\mathrm{CF}}=4.6,3.2 \mathrm{~Hz}, 1 \mathrm{C}\right), 19.8\left(\mathrm{dd}, J_{\mathrm{CF}}\right.$ $=3.8,2.3 \mathrm{~Hz}, 1 \mathrm{C}) ;{ }^{19} \mathrm{~F}$ NMR $\left(376 \mathrm{MHz}, \mathrm{CDCl}_{3}\right) \delta-109.7\left(\mathrm{~d}, J_{F F}=281.0 \mathrm{~Hz}, 1 \mathrm{~F}\right),-111.4\left(\mathrm{~d}, J_{F F}\right.$ $=281.1 \mathrm{~Hz}, 1 \mathrm{~F})$; IR (film) $v_{\max } 3509,2929,2860,1695,1589 \mathrm{~cm}^{-1}$; HRMS (ESI-TOF) $\mathrm{m} / z$ calcd for $\mathrm{C}_{11} \mathrm{H}_{11} \mathrm{C}_{12} \mathrm{~F}_{2} \mathrm{O}_{2}(\mathrm{M}+\mathrm{H})^{+}$283.0104, found 283.0095.
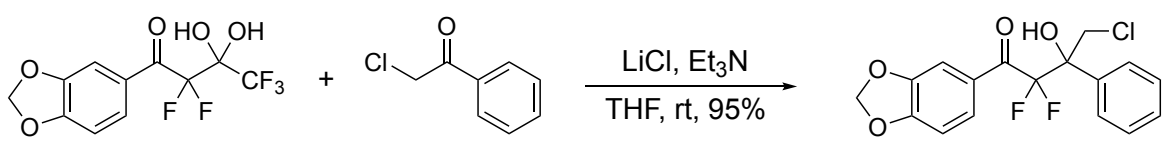

15

1-(Benzo[1,3]dioxol-5-yl)-4-chloro-2,2-difluoro-3-hydroxy-3-phenylbutan-1-one $15 . \quad$ See representative reaction procedure. A solution of 1-(benzo[1,3]dioxol-5-yl)-2,2,4,4,4-pentafluoro3,3-dihydroxybutan-1-one ${ }^{1}$ (100 mg, $0.3 \mathrm{mmol}$ ), 2-chloroacetophenone ( $98 \mathrm{mg}, 0.64 \mathrm{mmol}$ ), and $\mathrm{LiCl}(67 \mathrm{mg}, 1.6 \mathrm{mmol})$ in THF $(500 \mu \mathrm{L})$ was treated with $\mathrm{Et}_{3} \mathrm{~N}(53 \mu \mathrm{L}, 0.38 \mathrm{mmol}) . \mathrm{SiO}_{2}$ flash chromatography (19:1 hexanes/EtOAc) afforded the product 15 in 95\% yield $(107 \mathrm{mg})$ as a colorless oil: ${ }^{1} \mathrm{H}$ NMR $\left(500 \mathrm{MHz}, \mathrm{CDCl}_{3}\right) \delta 7.67(\mathrm{~d}, J=8.4 \mathrm{~Hz}, 1 \mathrm{H}), 7.56(\mathrm{~d}, J=7.1 \mathrm{~Hz}, 2 \mathrm{H}), 7.45$ $(\mathrm{s}, 1 \mathrm{H}), 7.44-7.35(\mathrm{~m}, 3 \mathrm{H}), 6.82(\mathrm{~d}, J=8.4 \mathrm{~Hz}, 1 \mathrm{H}), 6.05(\mathrm{~s}, 2 \mathrm{H}), 4.32(\mathrm{~d}, J=12.1 \mathrm{~Hz}, 1 \mathrm{H}), 4.29$ $(\mathrm{d}, J=11.7 \mathrm{~Hz}, 1 \mathrm{H}), 3.63(\mathrm{~s}, 1 \mathrm{H}) ;{ }^{13} \mathrm{C}$ DEPTQ NMR $\left(125 \mathrm{MHz}, \mathrm{CDCl}_{3}\right) \delta 188.5\left(\mathrm{dd}, J_{\mathrm{CF}}=29.5\right.$, 
$27.3 \mathrm{~Hz}, 1 \mathrm{C}), 153.1,148.0,136.0,129.0,128.5(2 \mathrm{C}), 128.2\left(\mathrm{dd}, J_{\mathrm{CF}}=5.4,3.7 \mathrm{~Hz}, 1 \mathrm{C}\right), 127.7$, $127.0(2 \mathrm{C}), 116.7\left(\mathrm{dd}, J_{\mathrm{CF}}=265.9,263.2 \mathrm{~Hz}, 1 \mathrm{C}\right), 110.2\left(\mathrm{dd}, J_{\mathrm{CF}}=4.1,2.8 \mathrm{~Hz}, 1 \mathrm{C}\right), 108.1,102.2$, $78.5\left(\mathrm{t}, J_{\mathrm{CF}}=24.8 \mathrm{~Hz}, 1 \mathrm{C}\right), 48.2\left(\mathrm{t}, J_{\mathrm{CF}}=4.1 \mathrm{~Hz}, 1 \mathrm{C}\right) ;{ }^{19} \mathrm{~F} \mathrm{NMR}\left(376 \mathrm{MHz}, \mathrm{CDCl}_{3}\right) \delta-106.3(\mathrm{~d}$, $\left.J_{F F}=272.1 \mathrm{~Hz}, 1 \mathrm{~F}\right),-107.6\left(\mathrm{~d}, J_{F F}=272.1 \mathrm{~Hz}, 1 \mathrm{~F}\right)$; IR (film) $v_{\max } 3504,2911,1680,1604,1444$, $1268 \mathrm{~cm}^{-1}$; HRMS (ESI-TOF) $\mathrm{m} / z$ calcd for $\mathrm{C}_{17} \mathrm{H}_{13} \mathrm{Cl}_{2} \mathrm{~F}_{2} \mathrm{O}_{4}(\mathrm{M}+\mathrm{Cl})^{-} 389.0159$, found 389.0162 .

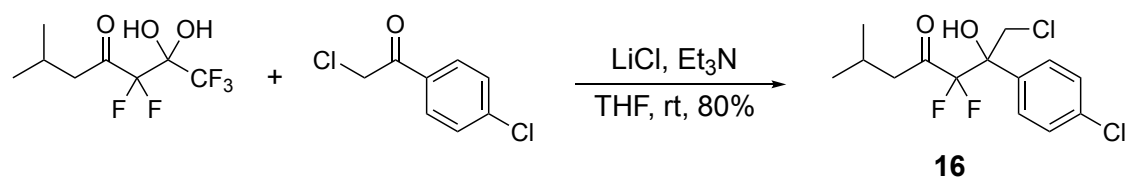

1-Chloro-2-(4-chlorophenyl)-3,3-difluoro-2-hydroxy-6-methylheptan-4-one $16 . \quad$ See representative reaction procedure. A solution of 1,1,1,3,3-pentafluoro-2,2-dihydroxy-6methylheptan-4-one ${ }^{1}$ (75 mg, $0.30 \mathrm{mmol}$ ), 2,4'-dichloroacetophenone (108 $\mathrm{mg}, 0.598 \mathrm{mmol}$ ), and $\mathrm{LiCl}(63 \mathrm{mg}, 1.5 \mathrm{mmol})$ in THF $(500 \mu \mathrm{L})$ was treated with $\mathrm{Et}_{3} \mathrm{~N}(50 \mu \mathrm{L}, 0.36 \mathrm{mmol}) . \mathrm{SiO}_{2}$ flash chromatography (19:1 hexanes/EtOAc) afforded the product 16 in $80 \%$ yield (70 $\mathrm{mg})$ as a colorless oil: ${ }^{1} \mathrm{H}$ NMR $\left(400 \mathrm{MHz}, \mathrm{CDCl}_{3}\right) \delta 7.44(\mathrm{~d}, J=8.4 \mathrm{~Hz}, 2 \mathrm{H}), 7.39(\mathrm{~d}, J=8.6 \mathrm{~Hz}, 2 \mathrm{H}), 4.21(\mathrm{~d}, J=$ $11.9 \mathrm{~Hz}, 1 \mathrm{H}), 4.13(\mathrm{~d}, J=11.9 \mathrm{~Hz}, 1 \mathrm{H}) .3 .34$ (br s, 1H), 2.58-2.41 (m, 2H), $2.10(\mathrm{~m}, 1 \mathrm{H}), 0.88(\mathrm{~d}$, $J=2.5 \mathrm{~Hz}, 3 \mathrm{H}), 0.86(\mathrm{~d}, J=2.8 \mathrm{~Hz}, 3 \mathrm{H}) ;{ }^{13} \mathrm{C} \mathrm{NMR}\left(100 \mathrm{MHz}, \mathrm{CDCl}_{3}\right) \delta 201.1\left(\mathrm{t}, J_{\mathrm{CF}}=29.1,28.0\right.$ $\mathrm{Hz}, 1 \mathrm{C}), 135.3,134.3,128.8(2 \mathrm{C}), 128.3(2 \mathrm{C}), 114.7\left(\mathrm{t}, J_{\mathrm{CF}}=264.1 \mathrm{~Hz}, 1 \mathrm{C}\right), 78.1$ (t, $J_{\mathrm{CF}}=24.9$ $\mathrm{Hz}, 1 \mathrm{C}), 48.0\left(\mathrm{t}, J_{\mathrm{CF}}=3.8 \mathrm{~Hz}, 1 \mathrm{C}\right), 47.7,23.5,22.4,22.4 ;{ }^{19} \mathrm{~F} \mathrm{NMR}\left(376 \mathrm{MHz}, \mathrm{CDCl}_{3}\right) \delta-115.1$ $\left(\mathrm{d}, J_{F F}=263.0 \mathrm{~Hz}, 1 \mathrm{~F}\right),-115.9\left(\mathrm{~d}, J_{F F}=262.6 \mathrm{~Hz}, 1 \mathrm{~F}\right)$; IR (film) $v_{\max } 3514,2965,2930,1741$, $1496 \mathrm{~cm}^{-1}$; HRMS (ESI-TOF) $m / z$ calcd for $\mathrm{C}_{14} \mathrm{H}_{16} \mathrm{C}_{13} \mathrm{~F}_{2} \mathrm{O}_{2}(\mathrm{M}+\mathrm{Cl})^{-} 359.0184$, found 359.0205 .

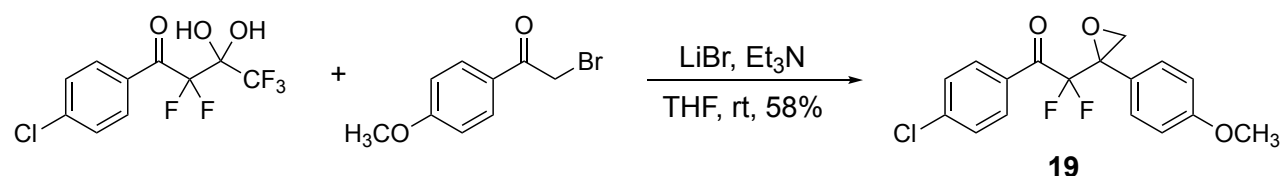

1-(4-Chlorophenyl)-2,2-difluoro-2-(2-(4-methoxyphenyl)oxiran-2-yl)ethan-1-one $19 . \quad$ See representative reaction procedure. A solution of 1-(4-chlorophenyl)-2,2,4,4,4-pentafluoro-3,3dihydroxybutan-1-one ${ }^{1}$ (75 mg, $0.25 \mathrm{mmol}$ ), 2-bromo-4'-methoxyacetophenone (65 mg, 0.49 $\mathrm{mmol})$, and $\mathrm{LiBr}(107 \mathrm{mg}, 1.23 \mathrm{mmol})$ in $\mathrm{THF}(500 \mu \mathrm{L})$ was treated with $\mathrm{Et}_{3} \mathrm{~N}(41 \mu \mathrm{L}, 0.30 \mathrm{mmol})$. $\mathrm{SiO}_{2}$ flash chromatography (19:1 hexanes/EtOAc) afforded the product 19 in $58 \%$ yield (48 $\mathrm{mg}$ ) as a colorless oil: ${ }^{1} \mathrm{H}$ NMR $\left(400 \mathrm{MHz}, \mathrm{CDCl}_{3}\right) \delta 7.97(\mathrm{~d}, J=8.2 \mathrm{~Hz}, 2 \mathrm{H}), 7.44(\mathrm{~d}, J=8.7 \mathrm{~Hz}, 2 \mathrm{H})$, $7.39(\mathrm{~d}, J=8.5 \mathrm{~Hz}, 2 \mathrm{H}), 6.86(\mathrm{~d}, J=8.8 \mathrm{~Hz}, 2 \mathrm{H}), 3.79(\mathrm{~s}, 3 \mathrm{H}), 3.42(\mathrm{~d}, J=5.2 \mathrm{~Hz}, 1 \mathrm{H}), 2.93(\mathrm{~d}$, $J=5.1 \mathrm{~Hz}, 1 \mathrm{H}) ;{ }^{13} \mathrm{C} \mathrm{NMR}\left(100 \mathrm{MHz}, \mathrm{CDCl}_{3}\right) \delta 187.5\left(\mathrm{t}, J_{\mathrm{CF}}=28.9 \mathrm{~Hz}, 1 \mathrm{C}\right), 160.3,141.2,131.8$ $\left(\mathrm{t}, J_{\mathrm{CF}}=3.1 \mathrm{~Hz}, 2 \mathrm{C}\right), 131.1,129.3(2 \mathrm{C}), 129.1(2 \mathrm{C}), 124.1,116.6\left(\mathrm{dd}, J_{\mathrm{CF}}=261.0,257.6 \mathrm{~Hz}, 1 \mathrm{C}\right)$, $114.0(2 \mathrm{C}), 59.1\left(\mathrm{dd}, J_{\mathrm{CF}}=32.0,28.9 \mathrm{~Hz}, 1 \mathrm{C}\right), 55.4,51.2\left(\mathrm{dd}, J_{\mathrm{CF}}=3.9,2.6 \mathrm{~Hz}, 1 \mathrm{C}\right)$; ${ }^{19} \mathrm{~F}$ NMR $\left(376 \mathrm{MHz}, \mathrm{CDCl}_{3}\right) \delta-104.88\left(\mathrm{~d}, J_{F F}=270.8 \mathrm{~Hz}, 1 \mathrm{~F}\right),-107.50\left(\mathrm{~d}, J_{F F}=270.8 \mathrm{~Hz}, 1 \mathrm{~F}\right)$; IR (film) $v_{\max } 3418,2965,2844,1675,1601,1256 \mathrm{~cm}^{-1}$; HRMS (ESI-TOF) $\mathrm{m} / z$ calcd for $\mathrm{C}_{17} \mathrm{H}_{14} \mathrm{ClF}_{2} \mathrm{O}_{3}$ $(\mathrm{M}+\mathrm{H})^{+}$339.0600, found 339.0603.

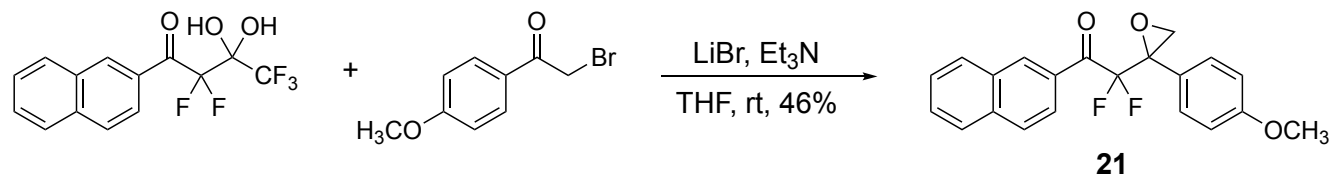

2,2-Difluoro-2-(2-(4-methoxyphenyl)oxiran-2-yl)-1-(naphthalen-2-yl)ethan-1-one 21. See representative reaction procedure. A solution of 2,2,4,4,4-pentafluoro-3,3-dihydroxy-1- 
(naphthalen-2-yl)butan-1-one ${ }^{1}$ (200 mg, $0.6 \mathrm{mmol}$ ), 2-bromo-4'-methoxyacetophenone (347 mg, $1.25 \mathrm{mmol})$, and $\mathrm{LiBr}(270 \mathrm{mg}, 3.1 \mathrm{mmol})$ in THF $(500 \mu \mathrm{L})$ was treated with $\mathrm{Et}_{3} \mathrm{~N}(104 \mu \mathrm{L}, 0.749$ mmol). $\mathrm{SiO}_{2}$ flash chromatography (19:1 hexanes/EtOAc) afforded the product 21 in $46 \%$ yield $(101 \mathrm{mg})$ as a colorless oil: ${ }^{1} \mathrm{H}$ NMR $\left(400 \mathrm{MHz}, \mathrm{CDCl}_{3}\right) \delta 8.65(\mathrm{~s}, 1 \mathrm{H}), 8.00(\mathrm{t}, J=9.2 \mathrm{~Hz}, 2 \mathrm{H})$, $7.91-7.83(\mathrm{~m}, 2 \mathrm{H}), 7.64(\mathrm{t}, J=7.6 \mathrm{~Hz}, 1 \mathrm{H}), 7.57(\mathrm{t}, J=7.5 \mathrm{~Hz}, 1 \mathrm{H}), 7.45(\mathrm{dd}, J=8.6,1.6 \mathrm{~Hz}, 2 \mathrm{H})$, $6.87(\mathrm{dd}, J=8.9,2.1 \mathrm{~Hz}, 2 \mathrm{H}), 3.78(\mathrm{~d}, J=2.2 \mathrm{~Hz}, 3 \mathrm{H}), 3.52(\mathrm{dd}, J=5.3,2.1 \mathrm{~Hz}, 1 \mathrm{H}), 3.00-2.96$ $(\mathrm{m}, 1 \mathrm{H}) ;{ }^{13} \mathrm{C} \mathrm{NMR}\left(100 \mathrm{MHz}, \mathrm{CDCl}_{3}\right) \delta 188.4\left(\mathrm{t}, J_{\mathrm{CF}}=28.6 \mathrm{~Hz}, 1 \mathrm{C}\right), 160.3,136.1,133.3\left(\mathrm{t}, J_{\mathrm{CF}}=\right.$ $4.0 \mathrm{~Hz}, 1 \mathrm{C}), 132.3,130.3,130.0,129.5,129.4$ (2C), 128.5, 127.8, 127.1, 124.9, 124.4, 116.8 (dd, $\left.J_{\mathrm{CF}}=260.7,256.9 \mathrm{~Hz}, 1 \mathrm{C}\right), 113.9(2 \mathrm{C}), 59.3\left(\mathrm{dd}, J_{\mathrm{CF}}=32.0,28.6 \mathrm{~Hz}, 1 \mathrm{C}\right), 55.4,51.3\left(\mathrm{dd}, J_{\mathrm{CF}}=\right.$ 3.9, $2.6 \mathrm{~Hz}, 1 \mathrm{C}) ;{ }^{19} \mathrm{~F}$ NMR $\left(376 \mathrm{MHz}, \mathrm{CDCl}_{3}\right) \delta-104.61\left(\mathrm{~d}, J_{F F}=270.4 \mathrm{~Hz}, 1 \mathrm{~F}\right),-107.18\left(\mathrm{~d}, J_{F F}\right.$ $=270.3 \mathrm{~Hz}, 1 \mathrm{~F}$ ); IR (film) $v_{\max } 3062,2934,1697,1516,1250 \mathrm{~cm}^{-1}$; HRMS (ESI-TOF) $\mathrm{m} / z$ calcd for $\mathrm{C}_{21} \mathrm{H}_{17} \mathrm{~F}_{2} \mathrm{O}_{3}(\mathrm{M}+\mathrm{H})^{+}$355.1146, found 355.1147.

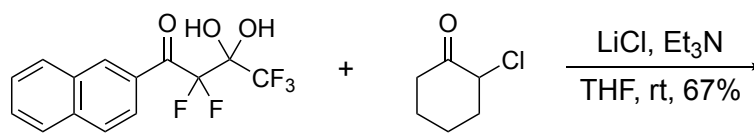

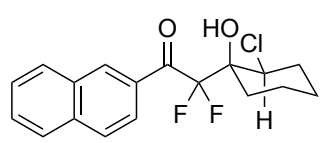

22

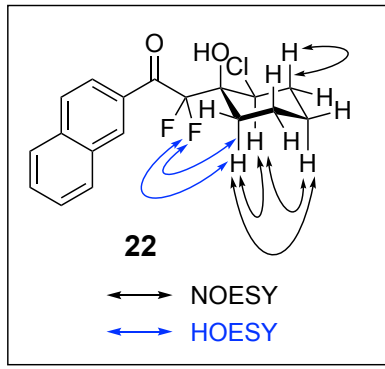
2-(2-Chloro-1-hydroxycyclohexyl)-2,2-difluoro-1-(naphthalen-2-yl)ethan-1-one 22. See
representative reaction procedure. A solution of 2,2,4,4,4-pentafluoro-3,3-dihydroxy-1(naphthalen-2-yl)butan-1-one ${ }^{1}(100 \mathrm{mg}, 0.3 \mathrm{mmol})$, chlorocyclohexanone $(178 \mu \mathrm{L}, 1.56 \mathrm{mmol})$, and $\mathrm{LiCl}(66 \mathrm{mg}, 1.6 \mathrm{mmol})$ in THF $(500 \mu \mathrm{L})$ was treated with $\mathrm{Et}_{3} \mathrm{~N}\left(53 \mu \mathrm{L}, 0.37 \mathrm{mmol}^{2} . \mathrm{SiO}_{2}\right.$ flash chromatography (19:1 hexanes/EtOAc) afforded the product 22 in $67 \%$ yield (106 $\mathrm{mg})$ as a colorless oil: ${ }^{1} \mathrm{H}$ NMR $\left(500 \mathrm{MHz}, \mathrm{CDCl}_{3}\right) \delta 8.71(\mathrm{~s}, 1 \mathrm{H}), 8.07(\mathrm{~d}, J=8.9 \mathrm{~Hz}, 1 \mathrm{H}), 8.00(\mathrm{~d}, J=8.2$ $\mathrm{Hz}, 1 \mathrm{H}), 7.89$ (t, $J=8.4 \mathrm{~Hz}, 2 \mathrm{H}), 7.64(\mathrm{ddd}, J=8.1,6.9,1.2 \mathrm{~Hz}, 1 \mathrm{H}), 7.58(\mathrm{ddd}, J=8.1,6.9,1.2$ $\mathrm{Hz}, 1 \mathrm{H}), 4.45(\mathrm{dd}, J=10.9,5.2 \mathrm{~Hz}, 1 \mathrm{H}), 3.60(\mathrm{~d}, J=2.1 \mathrm{~Hz}, 1 \mathrm{H}), 2.14(\mathrm{~d}, J=13.9 \mathrm{~Hz}, 1 \mathrm{H}), 2.10$ $1.98(\mathrm{~m}, 2 \mathrm{H}), 1.80-1.73(\mathrm{~m}, 2 \mathrm{H}), 1.68-1.59(\mathrm{~m}, 2 \mathrm{H}), 1.41(\mathrm{qt}, J=13.0,4.4 \mathrm{~Hz}, 1 \mathrm{H}) ;{ }^{13} \mathrm{C} \mathrm{NMR}$ $\left(125 \mathrm{MHz}, \mathrm{CDCl}_{3}\right) \delta 191.6\left(\mathrm{dd}, J_{\mathrm{CF}}=30.7,28.7 \mathrm{~Hz}, 1 \mathrm{C}\right), 136.0,133.4\left(\mathrm{dd}, J_{\mathrm{CF}}=6.3,4.0 \mathrm{~Hz}, 1 \mathrm{C}\right)$, $132.4,131.1,130.3,129.5,128.5,127.9,127.1,125.4,117.0\left(\mathrm{dd}, J_{\mathrm{CF}}=264.8,261.9 \mathrm{~Hz}, 1 \mathrm{C}\right), 76.7$ $\left(\mathrm{t}, J_{\mathrm{CF}}=23.6 \mathrm{~Hz}, 1 \mathrm{C}\right), 62.2,33.1,31.3\left(\mathrm{dd}, J_{\mathrm{CF}}=2.8,2.9 \mathrm{~Hz}, 1 \mathrm{C}\right), 25.4,19.6 ;{ }^{19} \mathrm{~F} \mathrm{NMR}(376 \mathrm{MHz}$, $\left.\mathrm{CDCl}_{3}\right) \delta-108.7\left(\mathrm{~d}, J_{F F}=279.0 \mathrm{~Hz}, 1 \mathrm{~F}\right),-110.5\left(\mathrm{~d}, J_{F F}=278.8 \mathrm{~Hz}, 1 \mathrm{~F}\right)$; IR (film) $v_{\max } 2474,2944$, 2867, 1720, 1626, $1449 \mathrm{~cm}^{-1}$; HRMS (ESI-TOF) $\mathrm{m} / z$ calcd for $\mathrm{C}_{18} \mathrm{H}_{17} \mathrm{ClF}_{2} \mathrm{NaO}_{2}(\mathrm{M}+\mathrm{Na})^{+}$ 361.0783, found 361.0764 .

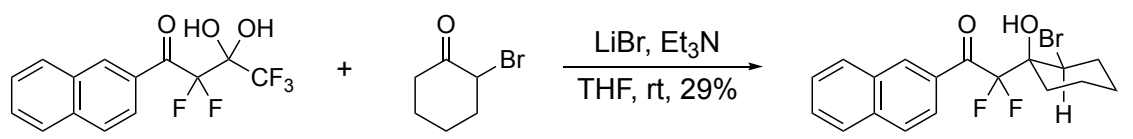

23

2-(2-Bromo-1-hydroxycyclohexyl)-2,2-difluoro-1-(naphthalen-2-yl)ethan-1-one $23 . \quad$ See representative reaction procedure. A solution of 2,2,4,4,4-pentafluoro-3,3-dihydroxy-1(naphthalen-2-yl)butan-1-one ${ }^{1}$ (373 mg, $\left.1.17 \mathrm{mmol}\right)$, bromocyclohexanone $(0.55 \mathrm{~mL}, 4.7 \mathrm{mmol})$, and $\mathrm{LiBr}(508 \mathrm{mg}, 5.58 \mathrm{mmol})$ in THF $(2 \mathrm{~mL})$ was treated with $\mathrm{Et}_{3} \mathrm{~N}(195 \mu \mathrm{L}, 1.40 \mathrm{mmol}) . \mathrm{SiO}_{2}$ flash chromatography (hexanes $\rightarrow 50: 1$ hexanes/EtOAc) afforded the product $\mathbf{2 3}$ in $29 \%$ yield $(131 \mathrm{mg})$ as a colorless oil: ${ }^{1} \mathrm{H}$ NMR $\left(400 \mathrm{MHz}, \mathrm{CDCl}_{3}\right) \delta 8.72(\mathrm{~s}, 1 \mathrm{H}), 8.08(\mathrm{~d}, J=8.7 \mathrm{~Hz}, 1 \mathrm{H})$, 
$8.00(\mathrm{~d}, J=8.1 \mathrm{~Hz}, 1 \mathrm{H}), 7.89(\mathrm{t}, J=8.6 \mathrm{~Hz}, 2 \mathrm{H}), 7.65(\mathrm{~m}, 1 \mathrm{H}), 7.58(\mathrm{~m}, 1 \mathrm{H}), 4.65(\mathrm{dd}, J=9.4$, $6.5 \mathrm{~Hz}, 1 \mathrm{H}), 3.49$ (d, $J=2.1 \mathrm{~Hz}, 1 \mathrm{H}), 2.25-2.17(\mathrm{~m}, 2 \mathrm{H}), 2.14(\mathrm{~d}, J=12.7 \mathrm{~Hz}, 1 \mathrm{H}), 1.85(\mathrm{~m}, 1 \mathrm{H})$, $1.79-1.63(\mathrm{~m}, 3 \mathrm{H}), 1.41(\mathrm{~m}, 1 \mathrm{H}) ;{ }^{13} \mathrm{C} \mathrm{NMR}\left(100 \mathrm{MHz}, \mathrm{CDCl}_{3}\right) \delta 191.3(\mathrm{dd}, J=39.1,28.7 \mathrm{~Hz})$, $136.0,133.5$ (dt, $J=6.2,4.0 \mathrm{~Hz}), 132.3,131.0,130.4,129.5(2 \mathrm{C}), 128.5,127.9,127.1,117.2$ (dd, $J=265.1,262.5 \mathrm{~Hz}), 76.2(\mathrm{t}, J=23.4 \mathrm{~Hz}), 56.0,34.4,31.7(\mathrm{t}, J=3.0 \mathrm{~Hz}), 26.4,19.8 ;{ }^{19} \mathrm{~F} \mathrm{NMR}$ $\left(376 \mathrm{MHz}, \mathrm{CDCl}_{3}\right) \delta-107.6\left(\mathrm{~d}, J_{F F}=278.3 \mathrm{~Hz}, 1 \mathrm{~F}\right),-109.6\left(\mathrm{~d}, J_{F F}=278.4 \mathrm{~Hz}, 1 \mathrm{~F}\right)$; IR (film) $v_{\max }$ $3520,2940,2864,1686,1627,1448 \mathrm{~cm}^{-1}$; HRMS (ESI-TOF) $m / z$ calcd for $\mathrm{C}_{18} \mathrm{H}_{16} \mathrm{BrF}_{2} \mathrm{O}_{2}(\mathrm{M}-$ $\mathrm{H})^{-}$381.0302, found 381.0327 .

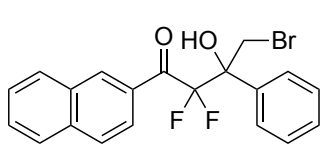

1

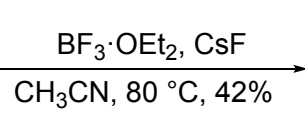

$\mathrm{CH}_{3} \mathrm{CN}, 80^{\circ} \mathrm{C}, 42 \%$

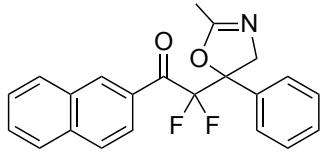

24

2,2-Difluoro-2-(2-methyl-5-phenyl-4,5-dihydrooxazol-5-yl)-1-(naphthalen-2-yl)ethan-1-one

24. ${ }^{3}$ To a solution of bromohydrin $1(140 \mathrm{mg}, 0.39 \mathrm{mmol})$ and $\mathrm{CsF}(314 \mathrm{mg}, 1.94 \mathrm{mmol})$ in $\mathrm{CH}_{3} \mathrm{CN}$ $(5 \mathrm{~mL}), \mathrm{BF}_{3} . \mathrm{Et}_{2} \mathrm{O}(239 \mu \mathrm{L}, 1.94 \mathrm{mmol})$ was added dropwise. The reaction was stirred for $16 \mathrm{~h}$ at $80{ }^{\circ} \mathrm{C}$ in an oil bath. The mixture was quenched with saturated aqueous $\mathrm{NH}_{4} \mathrm{Cl}(5 \mathrm{~mL})$ and extracted with $\mathrm{CH}_{2} \mathrm{Cl}_{2}(3 \times 5 \mathrm{~mL})$. The organics were dried over $\mathrm{Na}_{2} \mathrm{SO}_{4}$ and concentrated under reduced pressure. $\mathrm{SiO}_{2}$ flash chromatography (19:1 hexanes/EtOAc) afforded the product 24 in $42 \%$ yield $(60 \mathrm{mg})$ as a pale yellow oil: ${ }^{1} \mathrm{H} \mathrm{NMR}\left(500 \mathrm{MHz}, \mathrm{CDCl}_{3}\right) \delta 8.41(\mathrm{~s}, 1 \mathrm{H}), 7.91(\mathrm{~d}, J=$ $6.9 \mathrm{~Hz}, 1 \mathrm{H}), 7.87(\mathrm{~d}, J=8.2 \mathrm{~Hz}, 1 \mathrm{H}), 7.83(\mathrm{~d}, J=8.1 \mathrm{~Hz}, 1 \mathrm{H}), 7.80(\mathrm{~d}, J=8.9 \mathrm{~Hz}, 1 \mathrm{H}), 7.61(\mathrm{t}, J$ $=7.7 \mathrm{~Hz}, 1 \mathrm{H}), 7.55-7.50(\mathrm{~m}, 3 \mathrm{H}), 7.32(\mathrm{t}, J=7.3 \mathrm{~Hz}, 2 \mathrm{H}), 7.28(\mathrm{~d}, J=7.2 \mathrm{~Hz}, 1 \mathrm{H}), 5.24(\mathrm{~d}, J=$ $9.3 \mathrm{~Hz}, 1 \mathrm{H}), 4.57(\mathrm{~d}, J=9.3 \mathrm{~Hz}, 1 \mathrm{H}), 2.03(\mathrm{~s}, 3 \mathrm{H}) ;{ }^{13} \mathrm{C} \mathrm{NMR}\left(125 \mathrm{MHz}, \mathrm{CDCl}_{3}\right) \delta 190.1\left(\mathrm{t}, J_{C F}=\right.$ $29.1 \mathrm{~Hz}, 1 \mathrm{C}), 167.9,139.0,135.7,133.6\left(\mathrm{t}, J_{C F}=4.9 \mathrm{~Hz}, 1 \mathrm{C}\right), 132.1,130.7,130.2,129.2,128.4$, $128.3(2 \mathrm{C}), 127.9,127.9(2 \mathrm{C}), 127.6,126.7,125.3,117.7\left(\mathrm{t}, J_{C F}=262.8 \mathrm{~Hz}, 1 \mathrm{C}\right), 80.2\left(\mathrm{t}, J_{C F}=\right.$ $23.0 \mathrm{~Hz}, 1 \mathrm{C}), 74.5\left(\mathrm{t}, J_{C F}=3.6 \mathrm{~Hz}, 1 \mathrm{C}\right), 14.0 ;{ }^{19} \mathrm{~F}$ NMR $\left(376 \mathrm{MHz}, \mathrm{CDCl}_{3}\right) \delta-107.3\left(\mathrm{~d}, J_{F F}=\right.$ $264.4 \mathrm{~Hz}, 1 \mathrm{~F}$ ), -109.2 (d, $J_{F F}=264.6 \mathrm{~Hz}, 1 \mathrm{~F}$ ); IR (film) $v_{\max } 3318,2924,1663,1275 \mathrm{~cm}^{-1}$; HRMS (ESI-TOF) $m / z$ calcd for $\mathrm{C}_{22} \mathrm{H}_{18} \mathrm{~F}_{2} \mathrm{NO}_{2}(\mathrm{M}+\mathrm{H})^{+} 366.1306$, found 366.1322 .

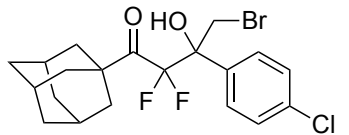

5

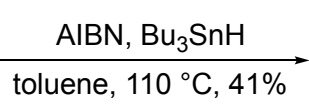

toluene, $110^{\circ} \mathrm{C}, 41 \%$

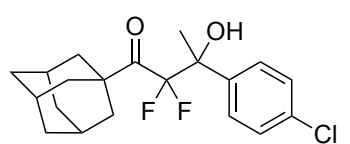

25

1-Adamantanyl)-3-(4-chlorophenyl)-2,2-difluoro-3-hydroxybutan-1-one 25. ${ }^{3}$ A solution of bromohydrin $5(50 \mathrm{mg}, 0.11 \mathrm{mmol})$ and $\mathrm{Bu}_{3} \mathrm{SnH}(36 \mu \mathrm{L}, 0.13 \mathrm{mmol})$ in toluene $(5 \mathrm{~mL})$ was heated to $110^{\circ} \mathrm{C}$ in an oil bath, then AIBN ( $\left.1 \mathrm{mg}, 0.06 \mathrm{mmol}\right)$ was added and the reaction was stirred for $16 \mathrm{~h}$. The mixture was concentrated under reduced pressure. $\mathrm{SiO}_{2}$ flash chromatography (19:1 hexanes/EtOAc) afforded the product 25 in $41 \%$ yield $(17 \mathrm{mg})$ as a colorless solid: $\mathrm{mp} 98-100{ }^{\circ} \mathrm{C}$; ${ }^{1} \mathrm{H}$ NMR $\left(400 \mathrm{MHz}, \mathrm{CDCl}_{3}\right) \delta 7.44(\mathrm{~d}, J=8.0 \mathrm{~Hz}, 2 \mathrm{H}), 7.32(\mathrm{~d}, J=8.7 \mathrm{~Hz}, 2 \mathrm{H}), 4.34(\mathrm{~s}, 1 \mathrm{H}), 1.99$ $(\mathrm{s}, 3 \mathrm{H}), 1.86-1.68(\mathrm{~m}, 9 \mathrm{H}), 1.67-1.61(\mathrm{~m}, 6 \mathrm{H}) ;{ }^{13} \mathrm{C}$ DEPTQ NMR $\left(100 \mathrm{MHz}, \mathrm{CDCl}_{3}\right) \delta 208.0(\mathrm{dd}$, $\left.J_{\mathrm{CF}}=30.7,27.5 \mathrm{~Hz}, 1 \mathrm{C}\right), 139.3\left(\mathrm{~d}, J_{\mathrm{CF}}=2.6 \mathrm{~Hz}, 1 \mathrm{C}\right), 134.1,128.3(2 \mathrm{C}), 128.1(2 \mathrm{C}), 115.7\left(\mathrm{t}, J_{\mathrm{CF}}\right.$ $=267.4 \mathrm{~Hz}, 1 \mathrm{C}), 76.6\left(\mathrm{t}, J_{\mathrm{CF}}=24.2 \mathrm{~Hz}, 1 \mathrm{C}\right), 36.8(3 \mathrm{C}), 36.4(3 \mathrm{C}), 29.9,27.7(3 \mathrm{C}), 23.6\left(\mathrm{dd}, J_{\mathrm{CF}}=\right.$ $3.7,2.4 \mathrm{~Hz}, 1 \mathrm{C}) ;{ }^{19} \mathrm{~F}$ NMR $\left(376 \mathrm{MHz}, \mathrm{CDCl}_{3}\right) \delta-108.79\left(\mathrm{~d}, J_{F F}=293.6 \mathrm{~Hz}, 1 \mathrm{~F}\right),-112.87\left(\mathrm{~d}, J_{F F}\right.$ $=294.5 \mathrm{~Hz}, 1 \mathrm{~F}$ ); IR (film) $v_{\max } 2912,2853,1720,1498 \mathrm{~cm}^{-1}$; HRMS (ESI-TOF) $\mathrm{m} / \mathrm{z}$ calcd for $\mathrm{C}_{20} \mathrm{H}_{23} \mathrm{Cl}_{2} \mathrm{~F}_{2} \mathrm{O}_{2}(\mathrm{M}+\mathrm{Cl})^{-}$403.1043, found 403.1070. 


\section{References}

1. Han, C.; Kim, E. H.; Colby, D. A. Cleavage of Carbon-Carbon Bonds through the Mild Release of Trifluoroacetate: Generation of $\alpha, \alpha$-Difluoroenolates for Aldol Reactions. J. Am. Chem. Soc. 2011, 130, 5802-5805.

2. Li, W.; Zhu, X.; Mao, H.; Tang, Z.; Cheng, Y.; Zhu, C. Visible-Light-Induced Direct C(sp3)$\mathrm{H}$ Difluoromethylation of Tetrahydroisoquinolines with the in situ Generated Difluoroenolates. Chem. Commun. 2014, 50, 7521-7523.

3. Sowaileh, M. F. Fluorinated Organic Molecules for Medicinal Applications. Ph.D. Dissertation, University of Mississippi: University, MS, 38677. 


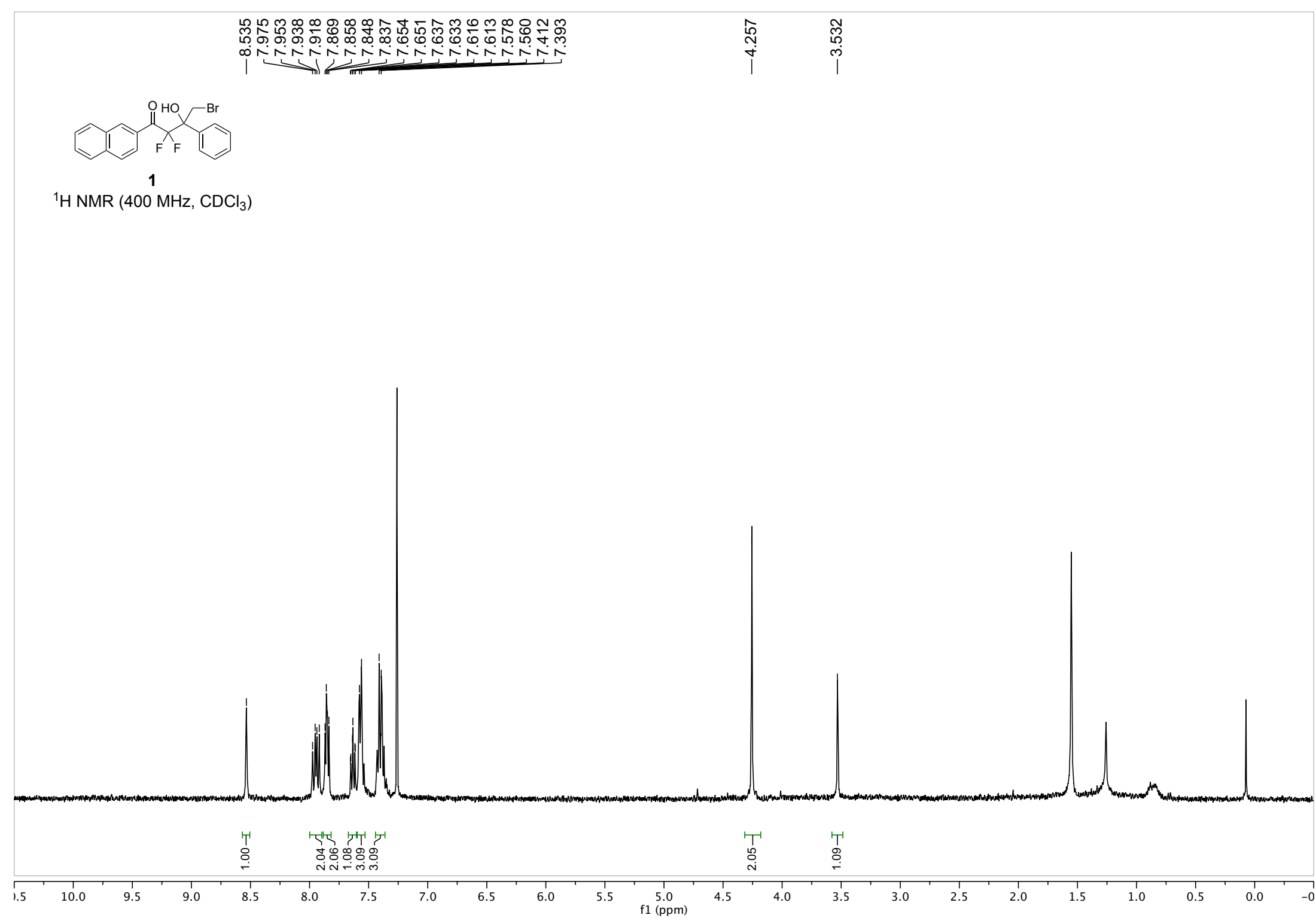




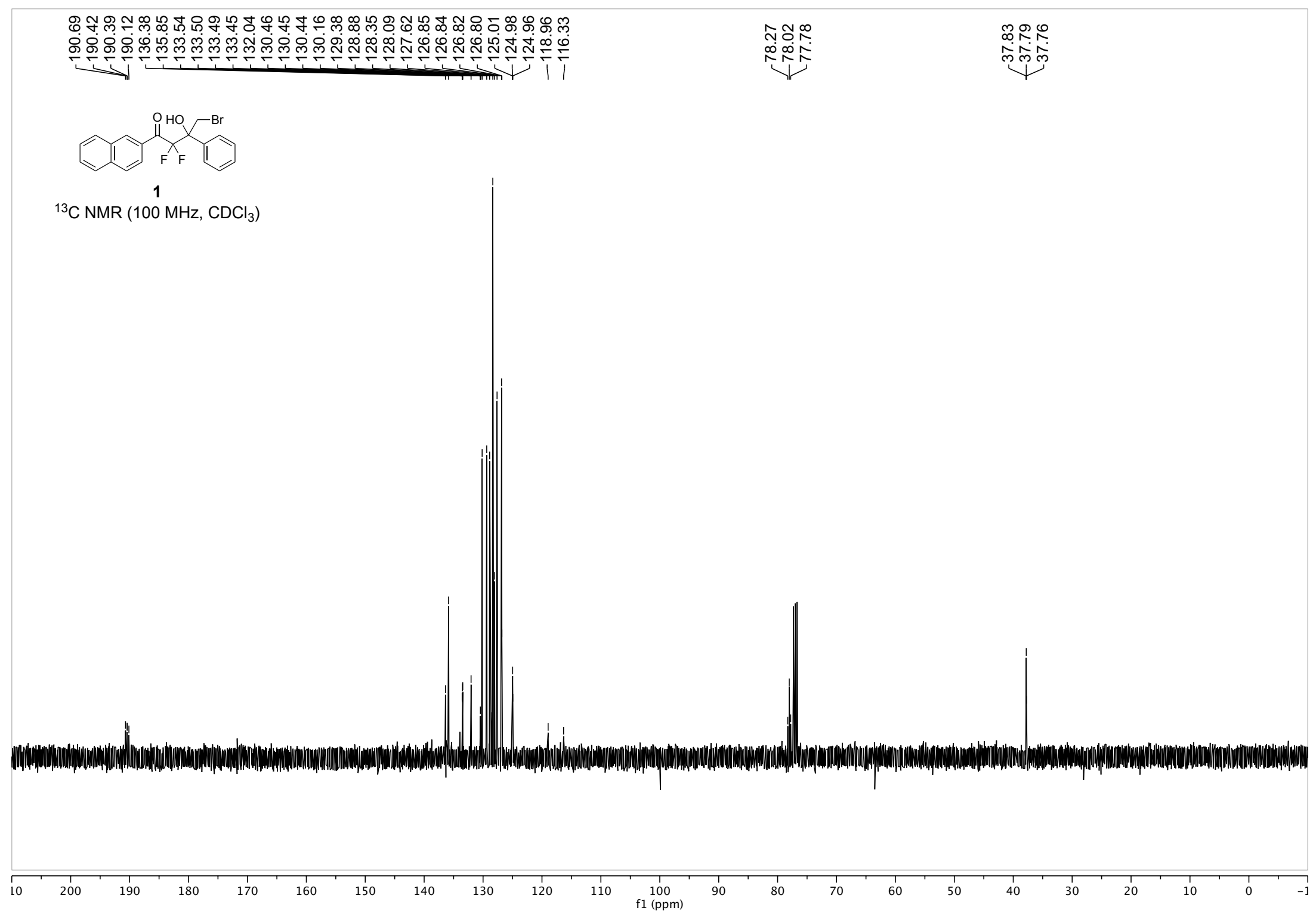



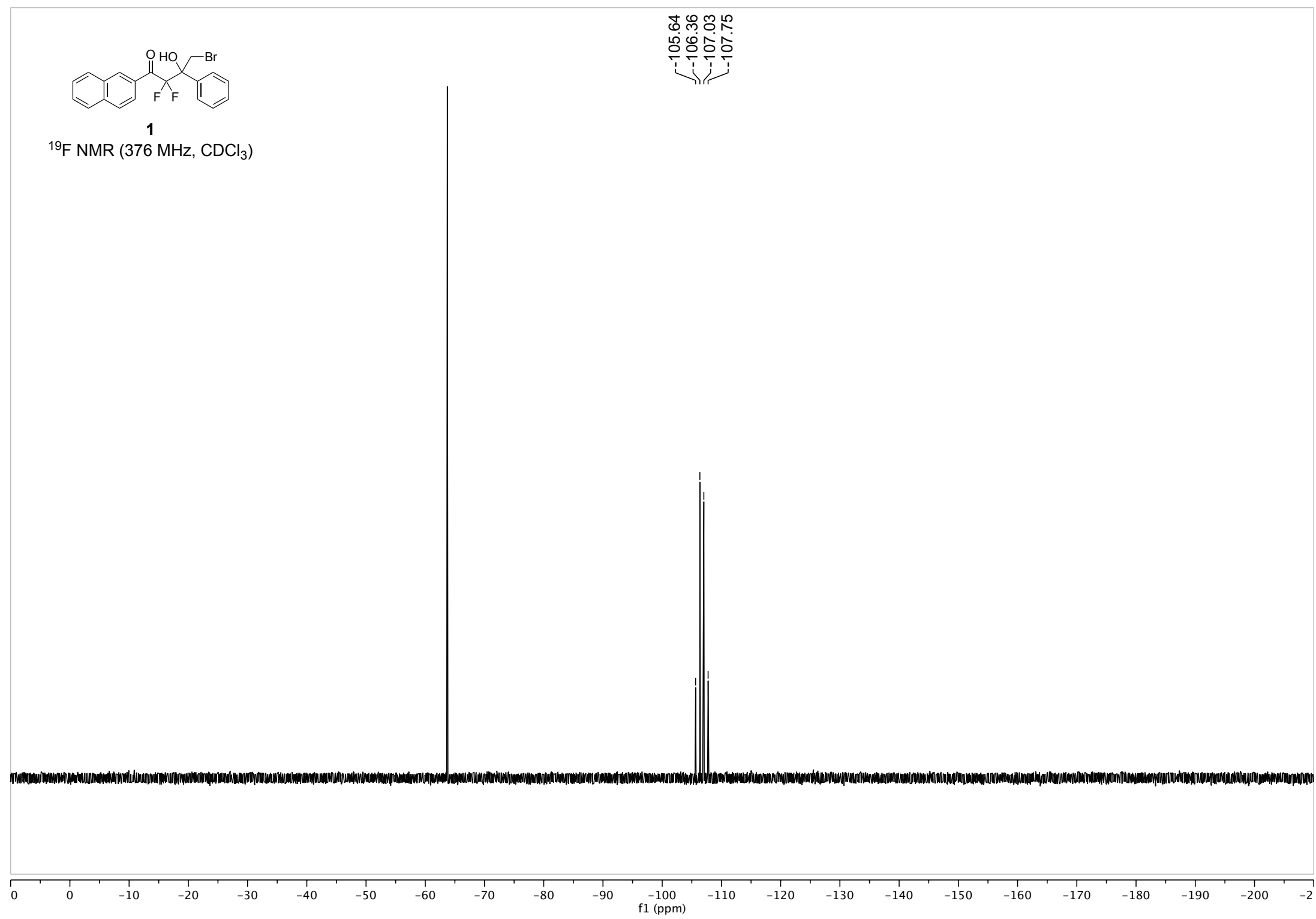


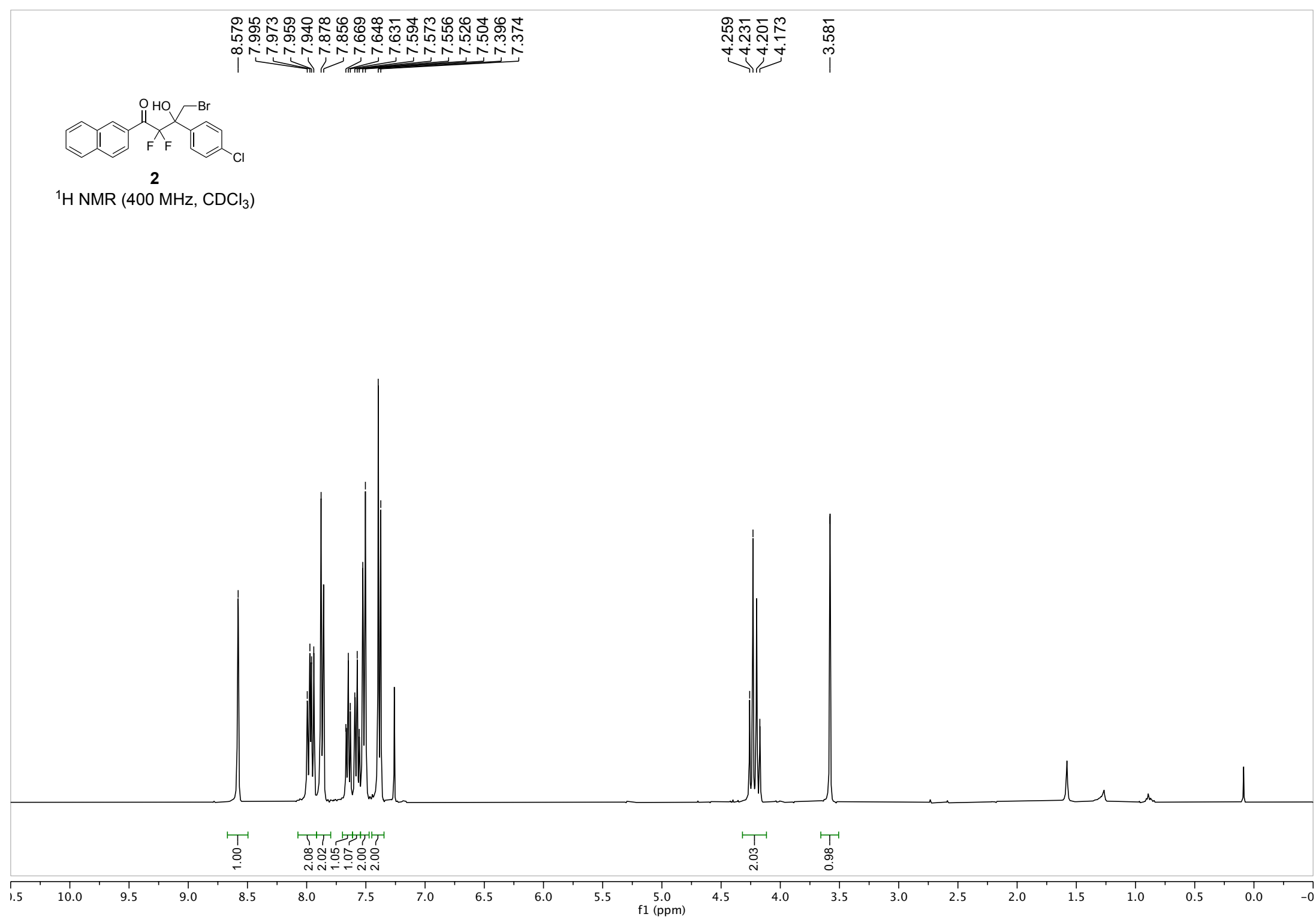




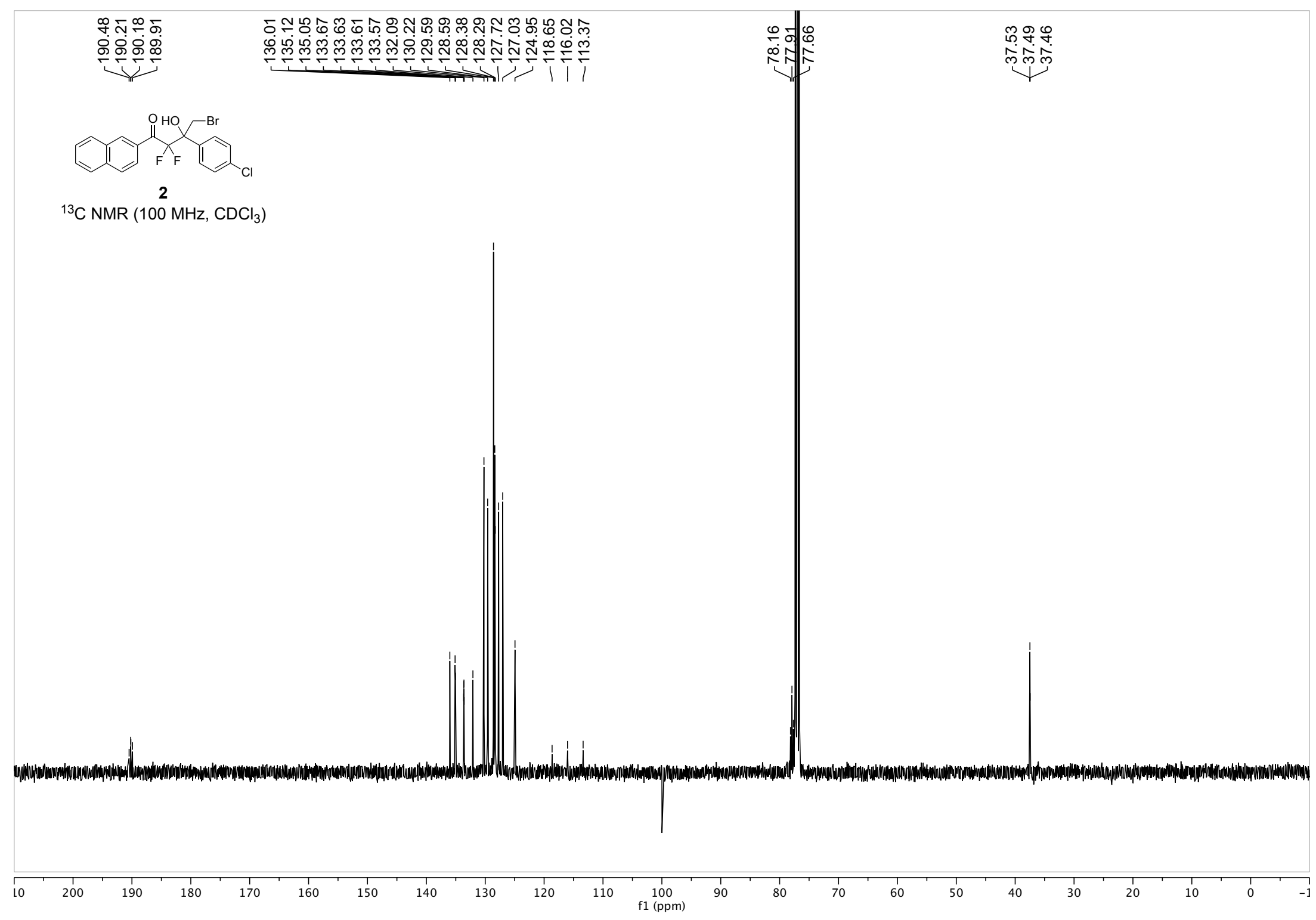


จุ๊

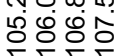

2

눈

${ }^{19} \mathrm{~F} \mathrm{NMR}\left(376 \mathrm{MHz}, \mathrm{CDCl}_{3}\right)$
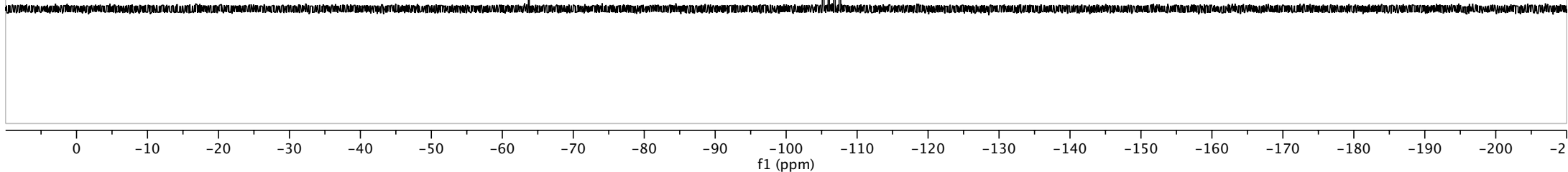


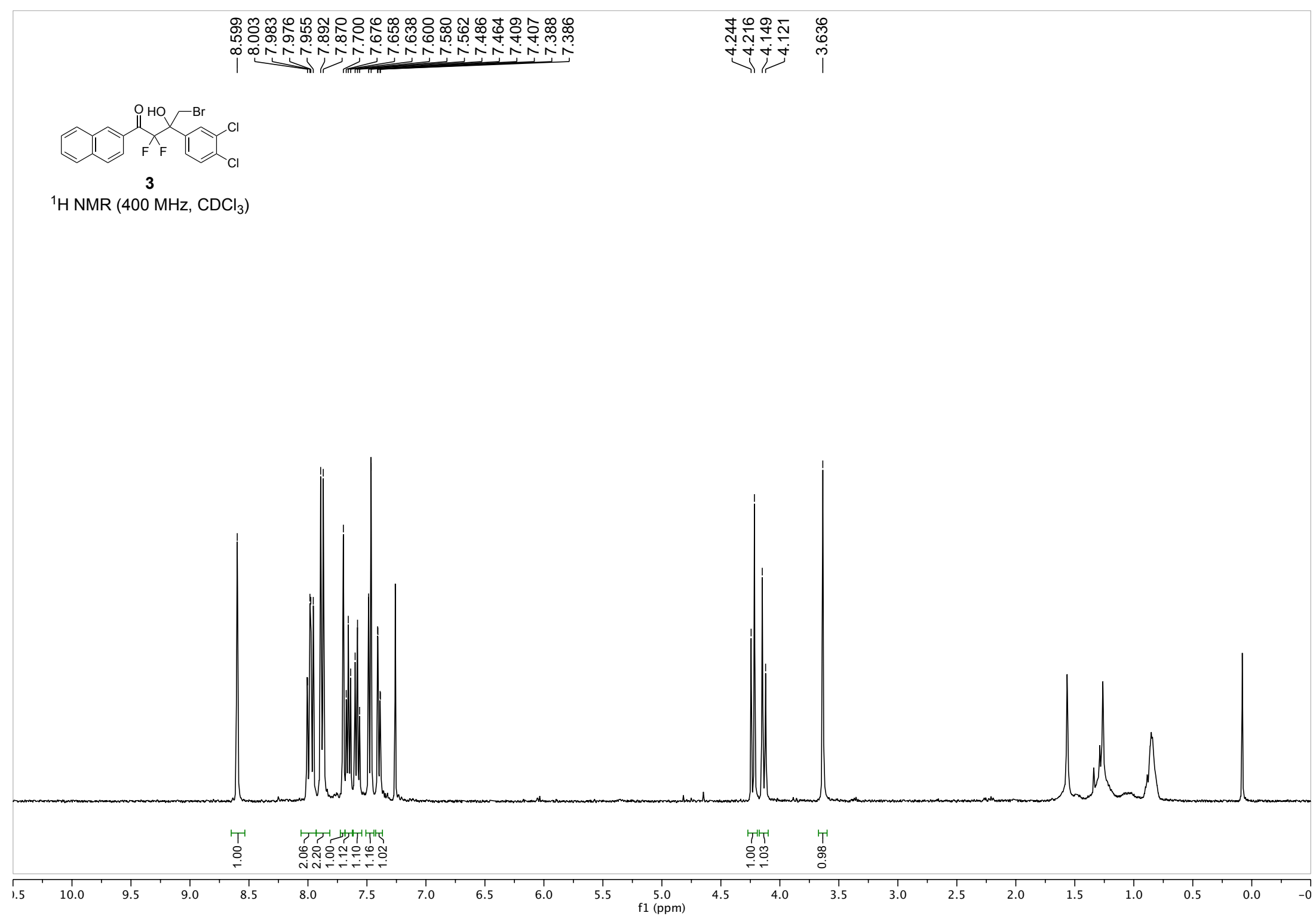




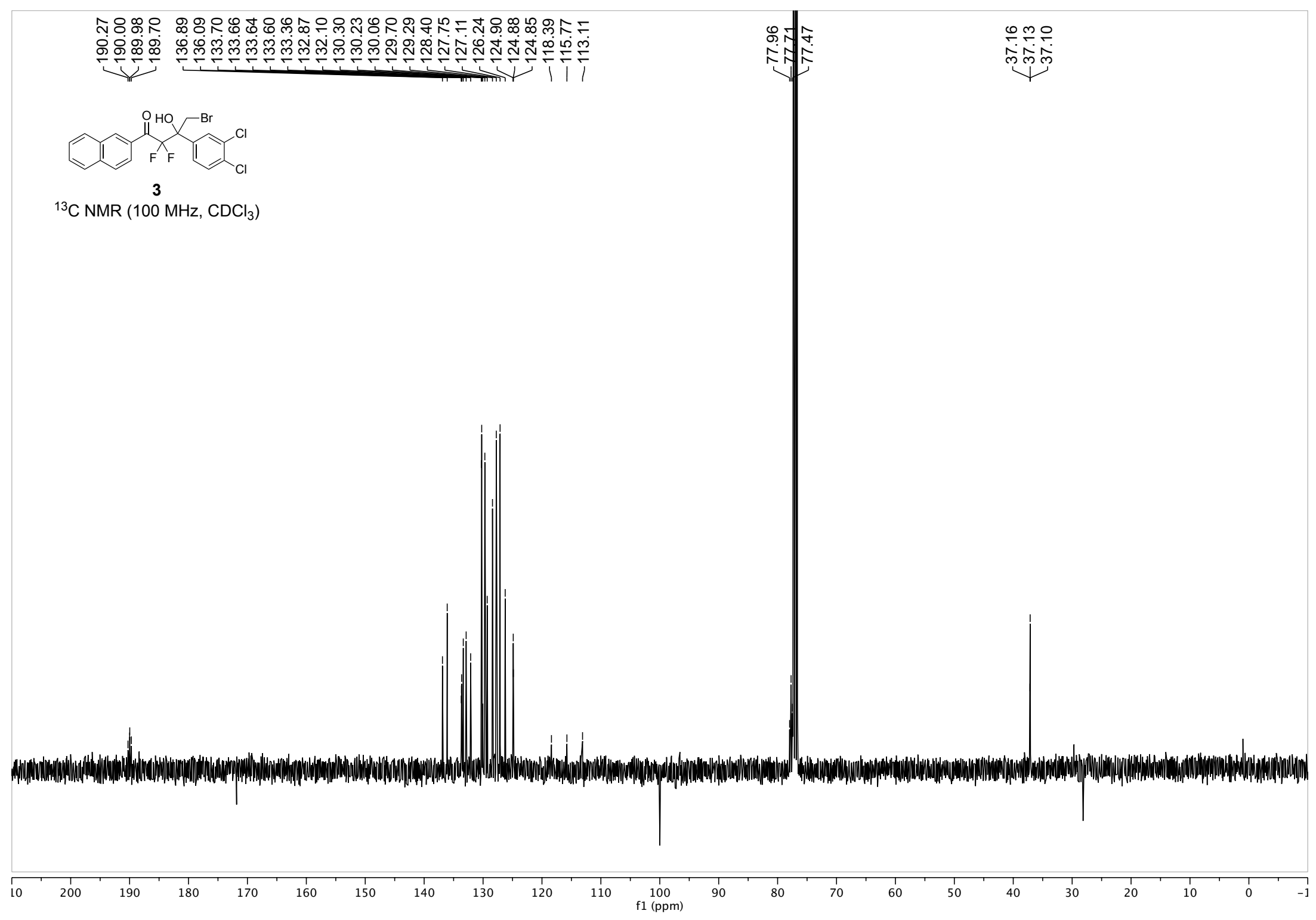




\section{๑ึิ์}

(l)

훙응흥

${ }^{19} \mathrm{~F}$ NMR $\left(376 \mathrm{MHz}, \mathrm{CDCl}_{3}\right)$
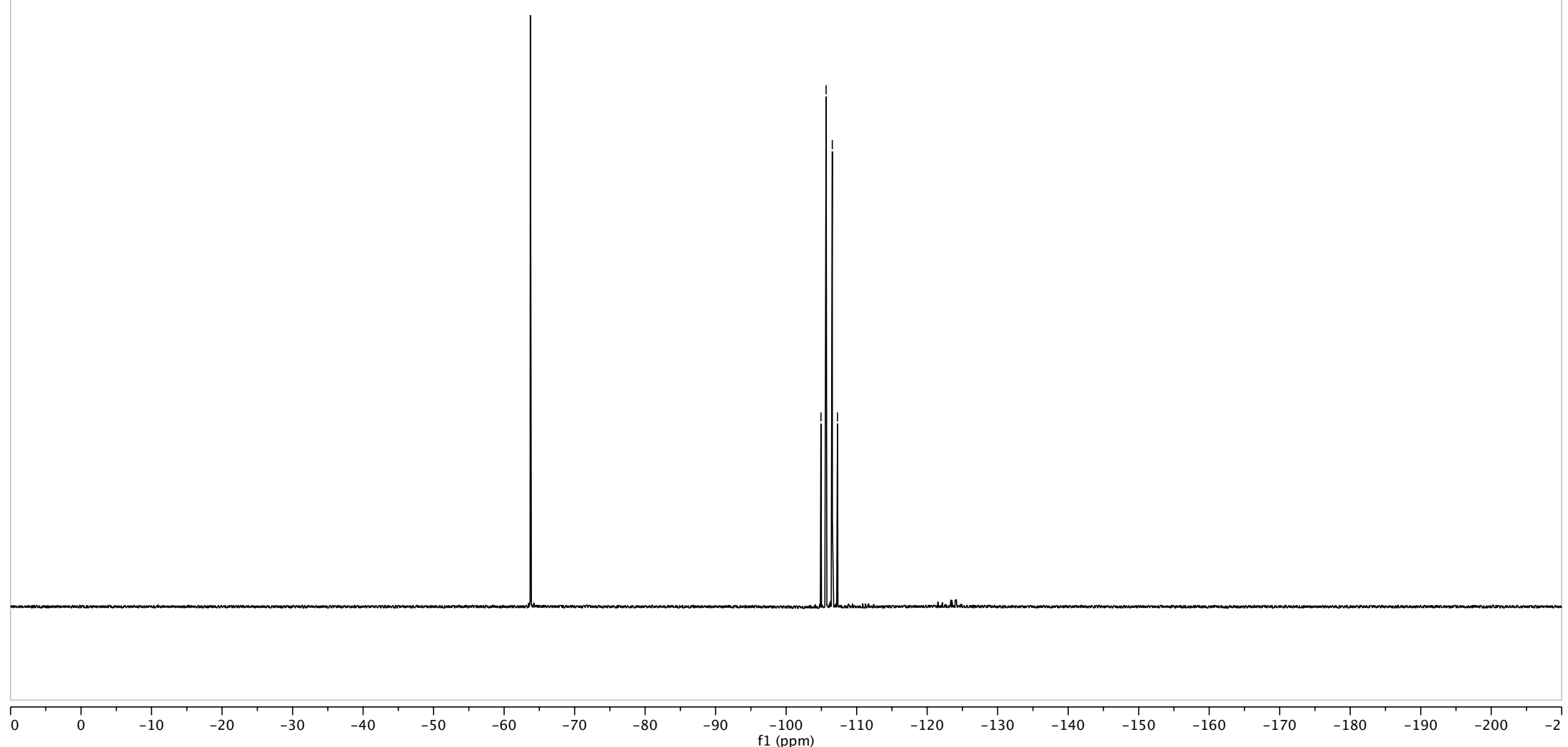


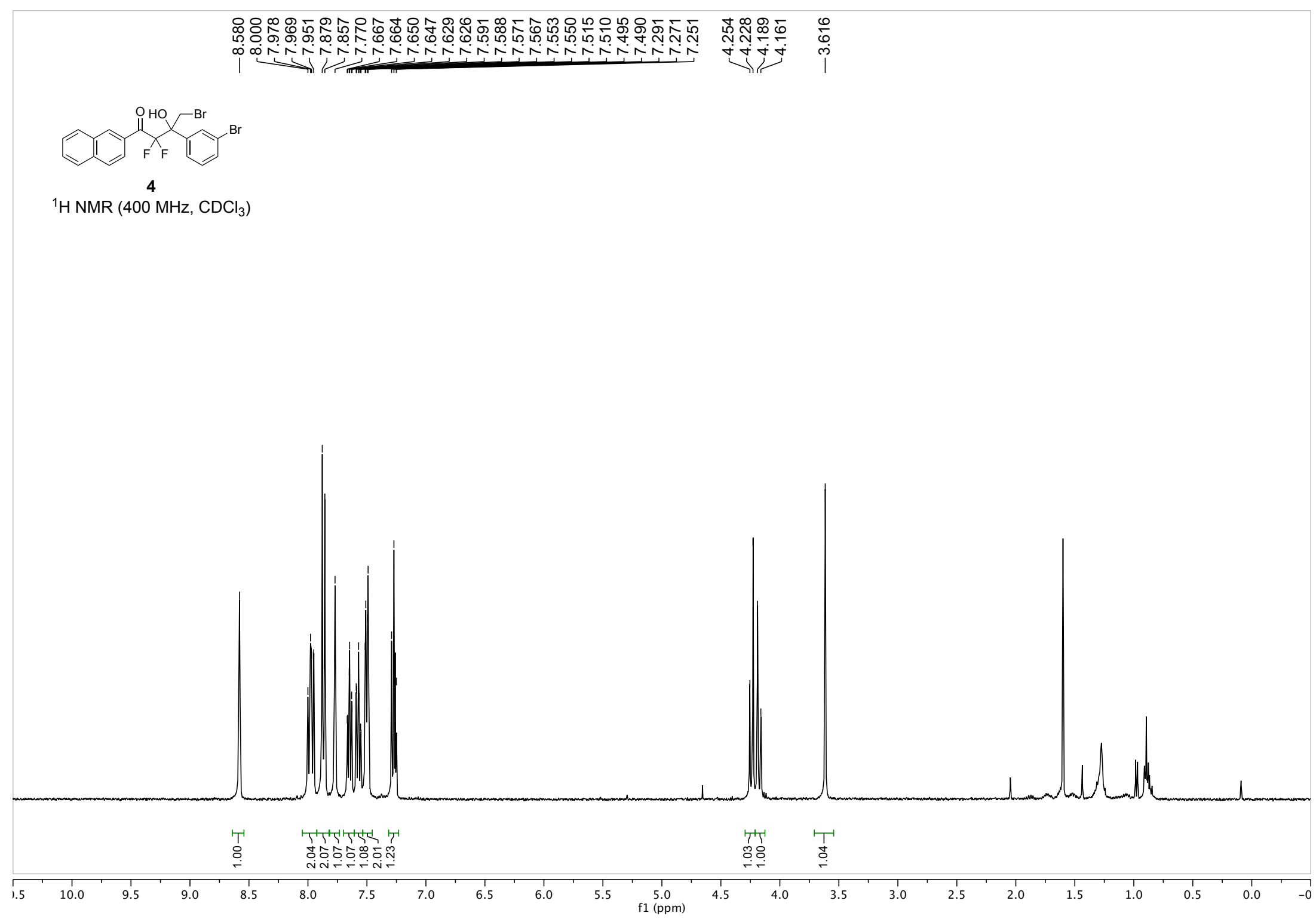




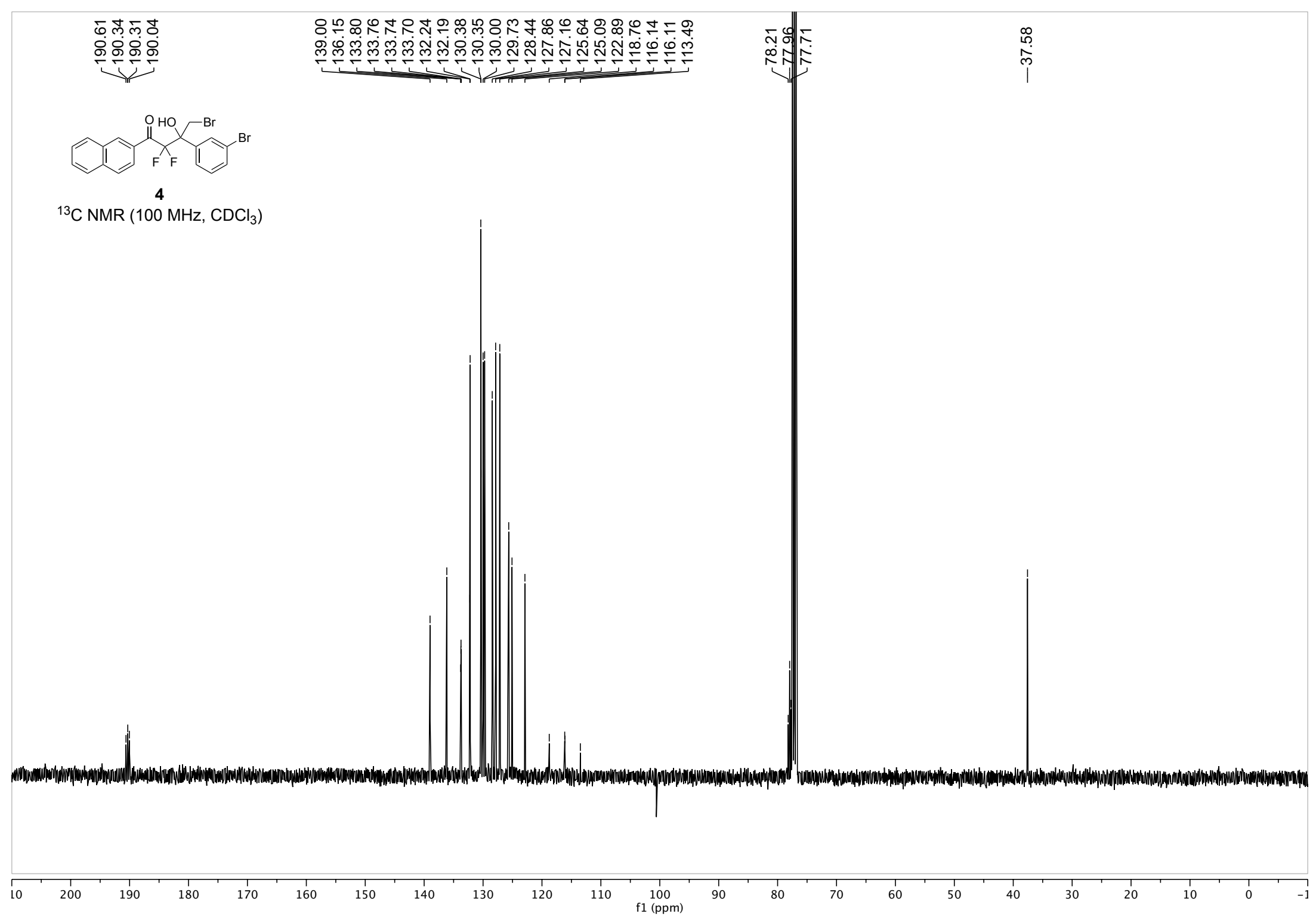




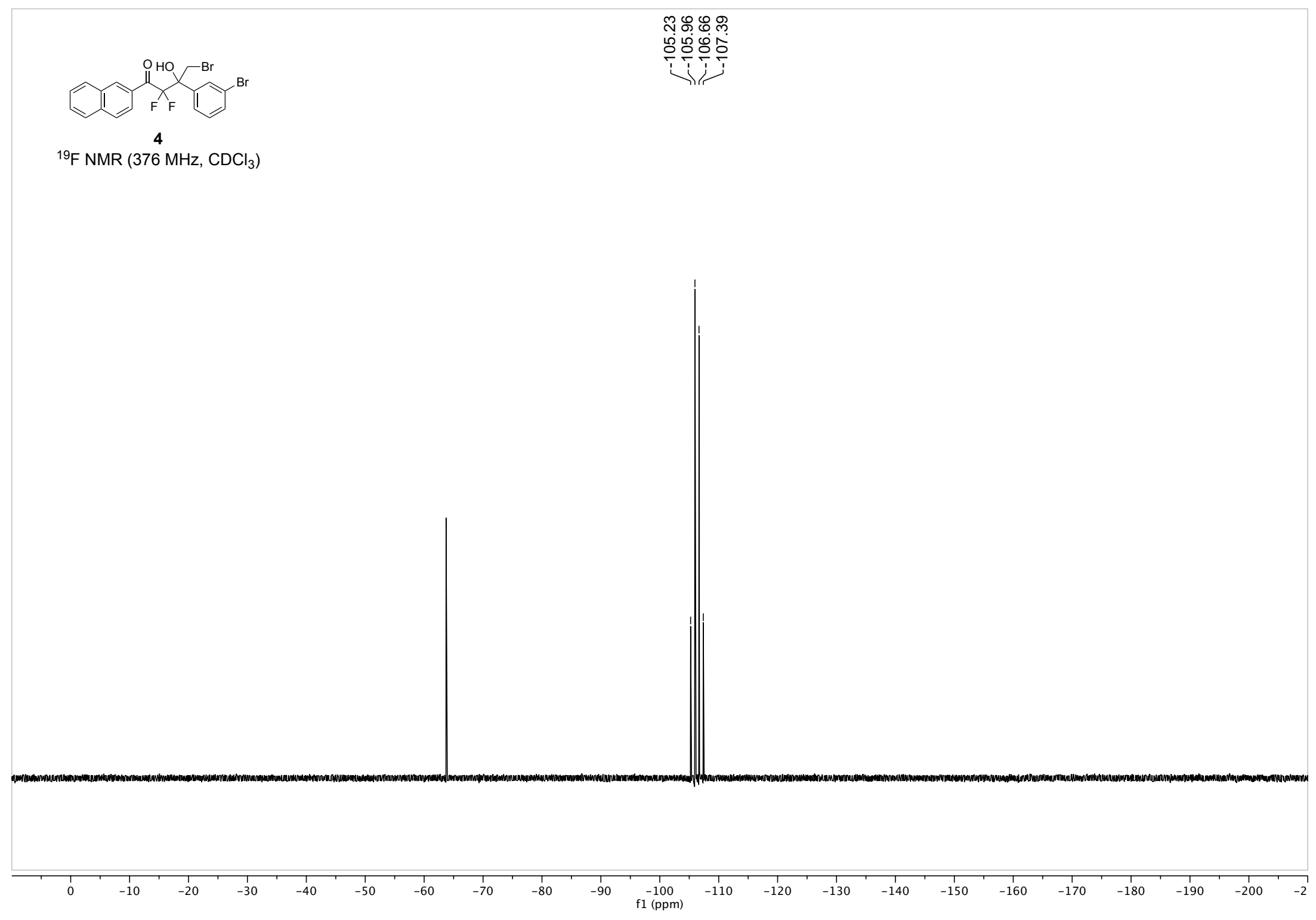




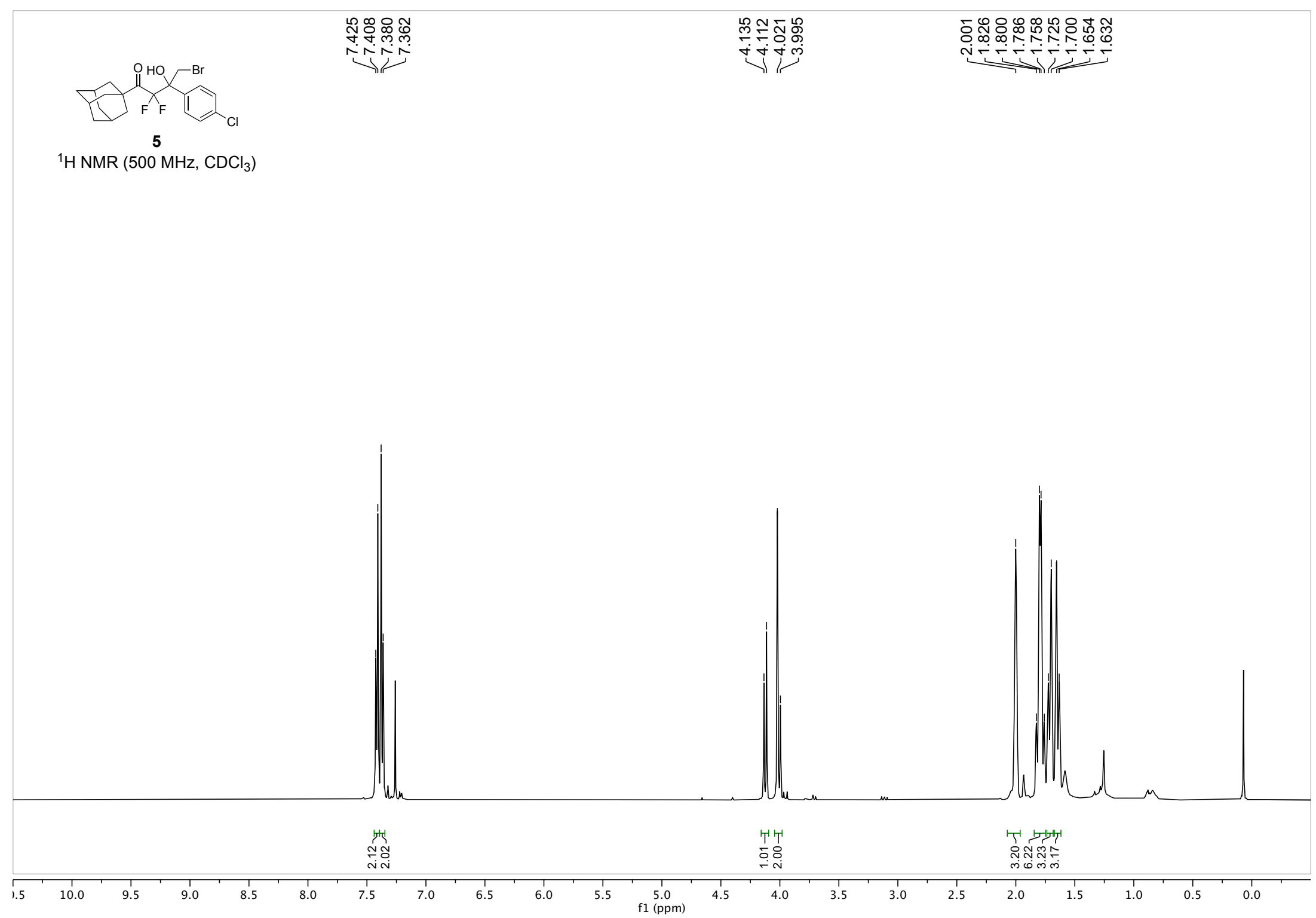




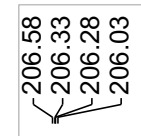

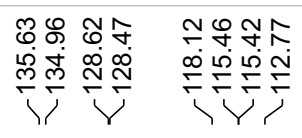

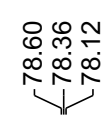

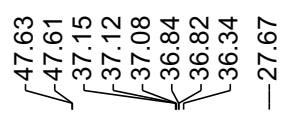

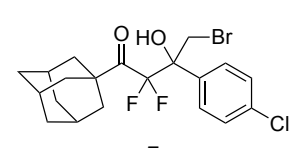

${ }^{13} \mathrm{C}$ NMR $\left(100 \mathrm{MHz}, \mathrm{CDCl}_{3}\right)$

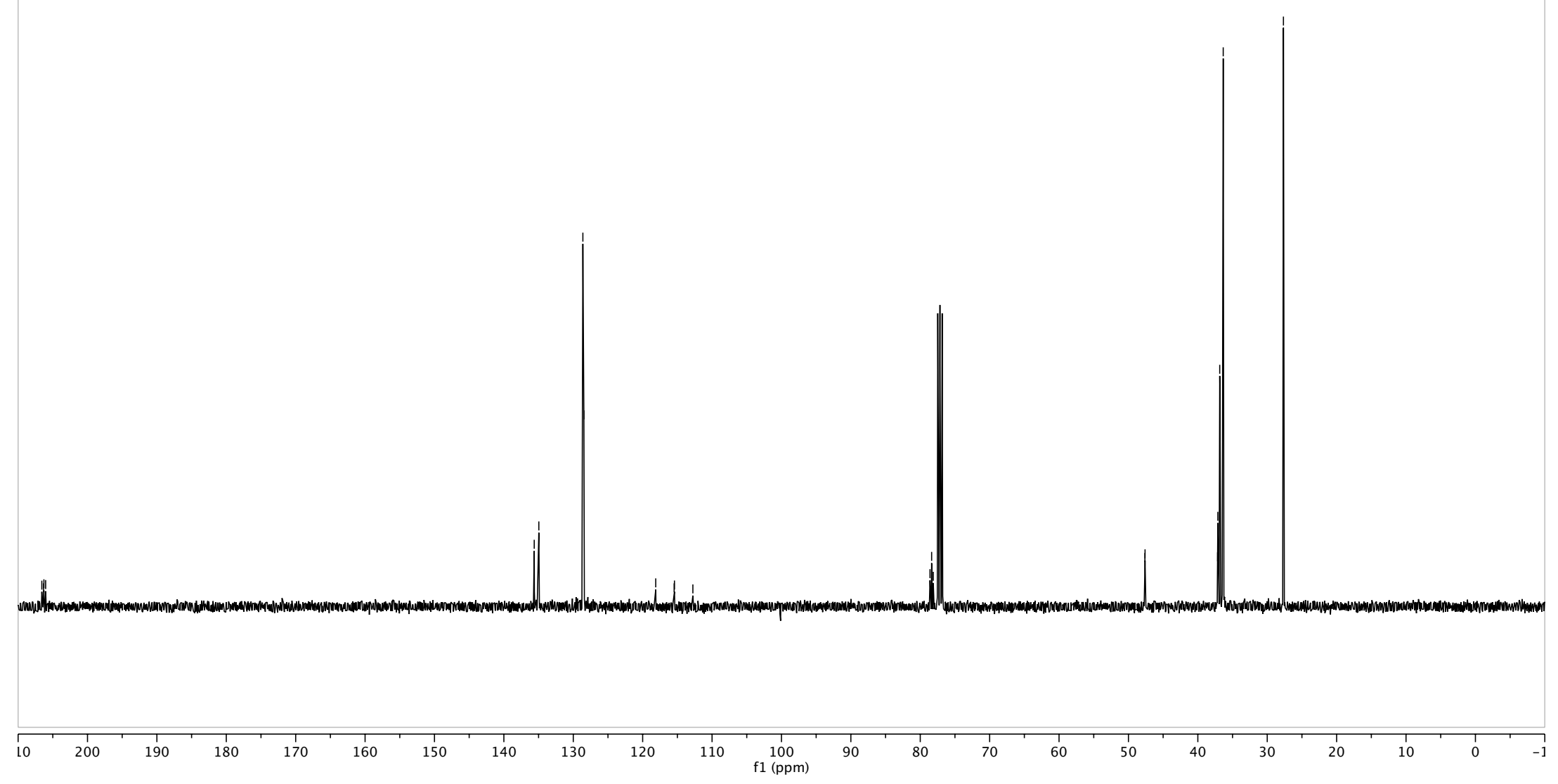

นั่ 


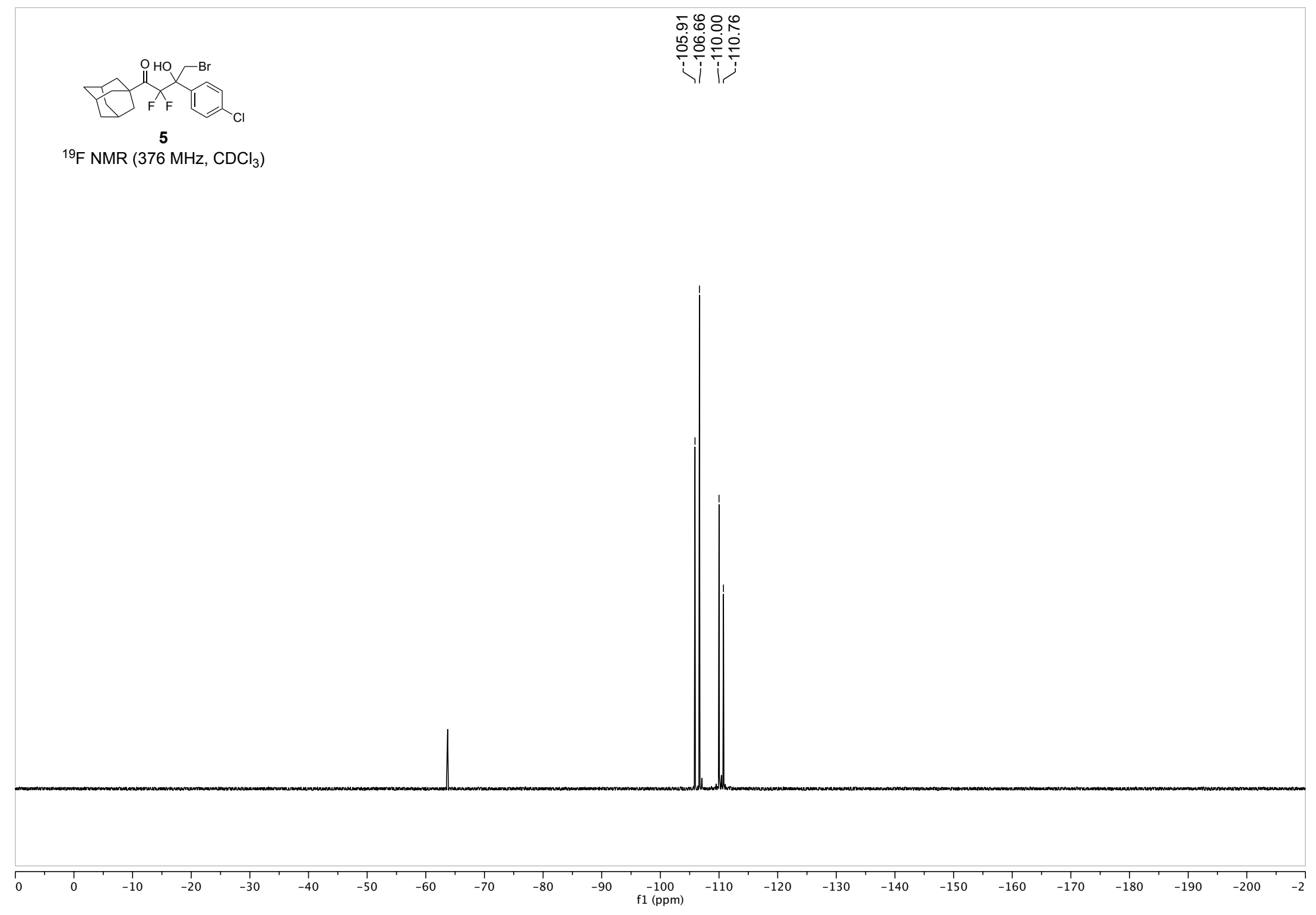

นั 


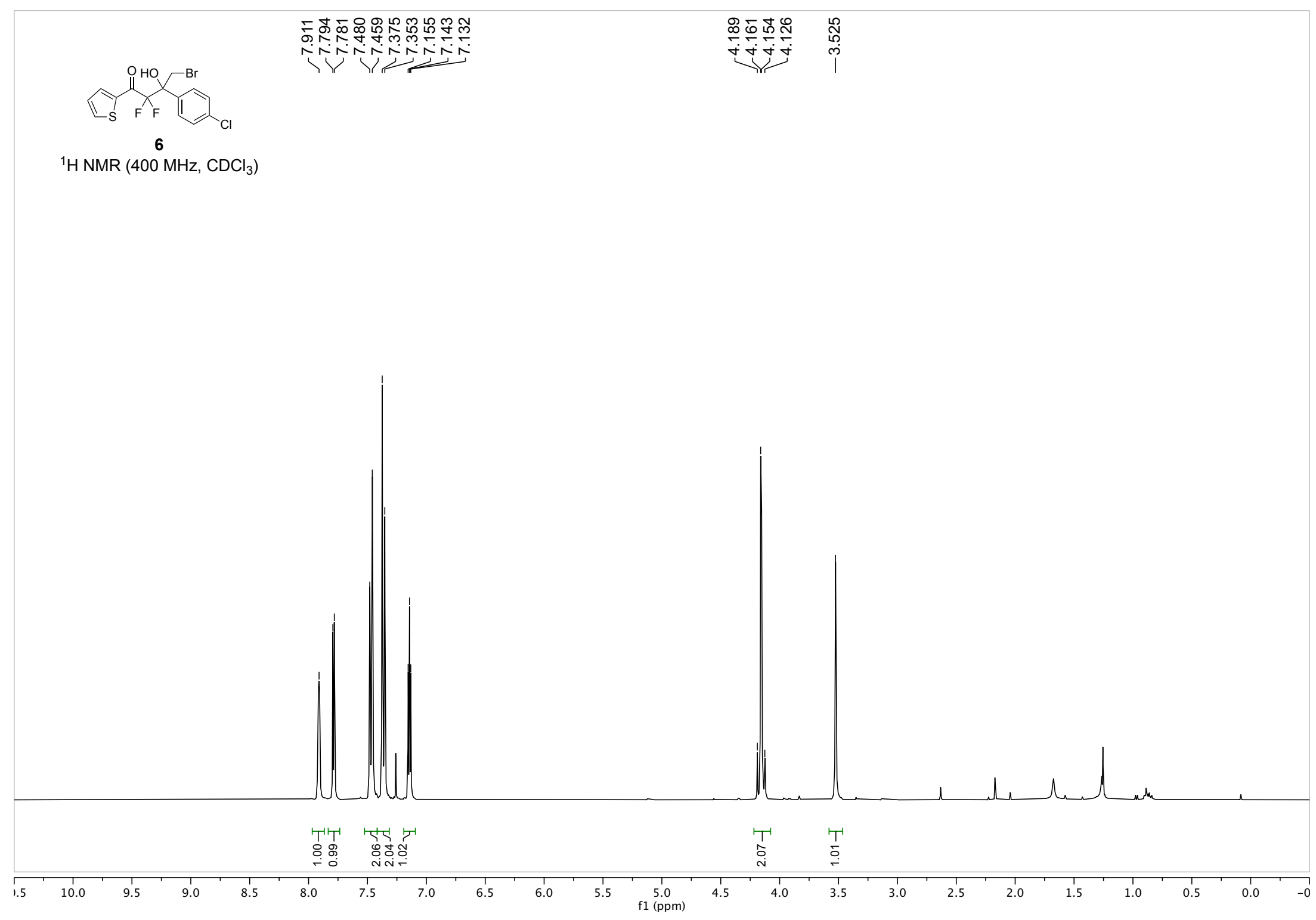




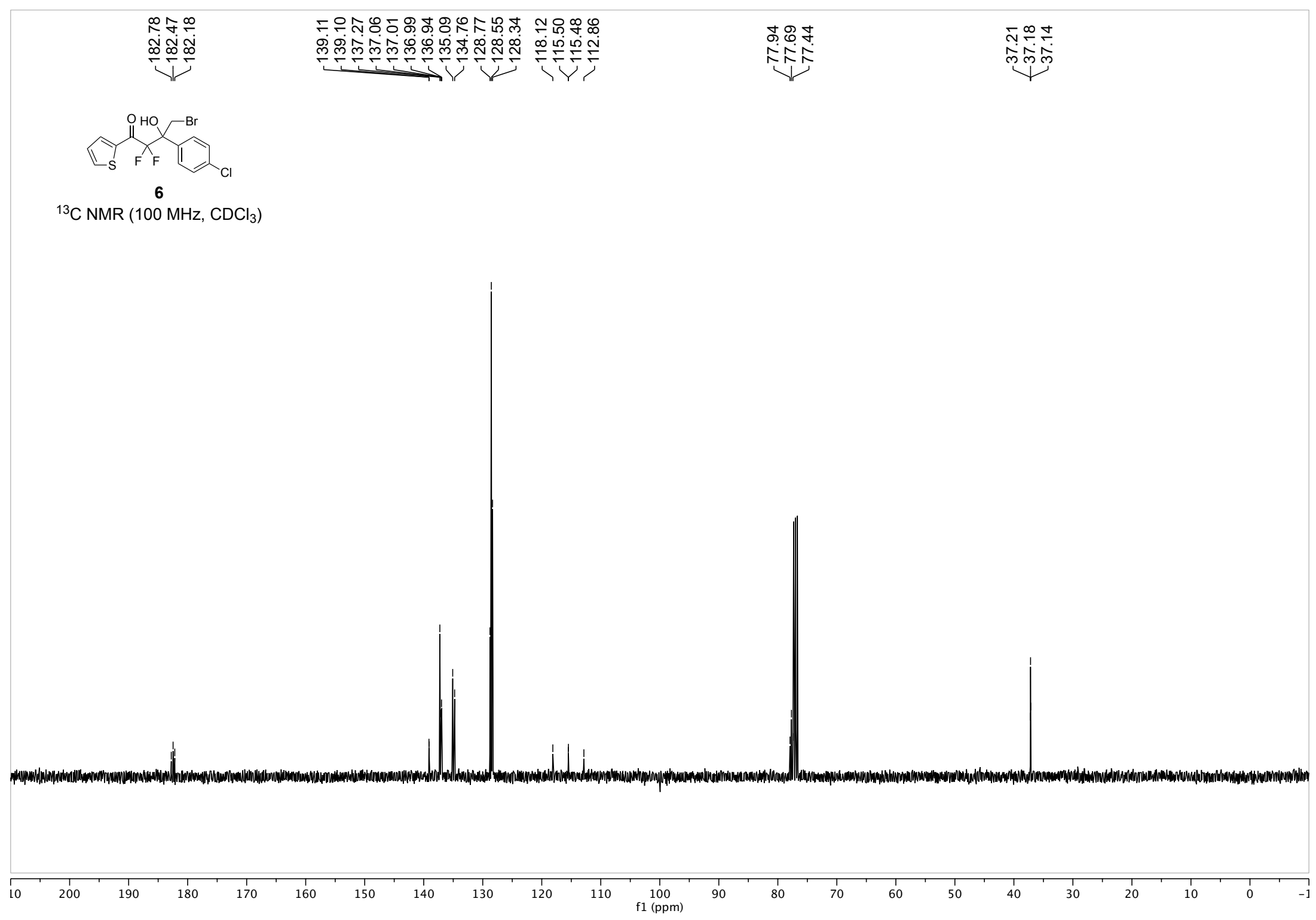




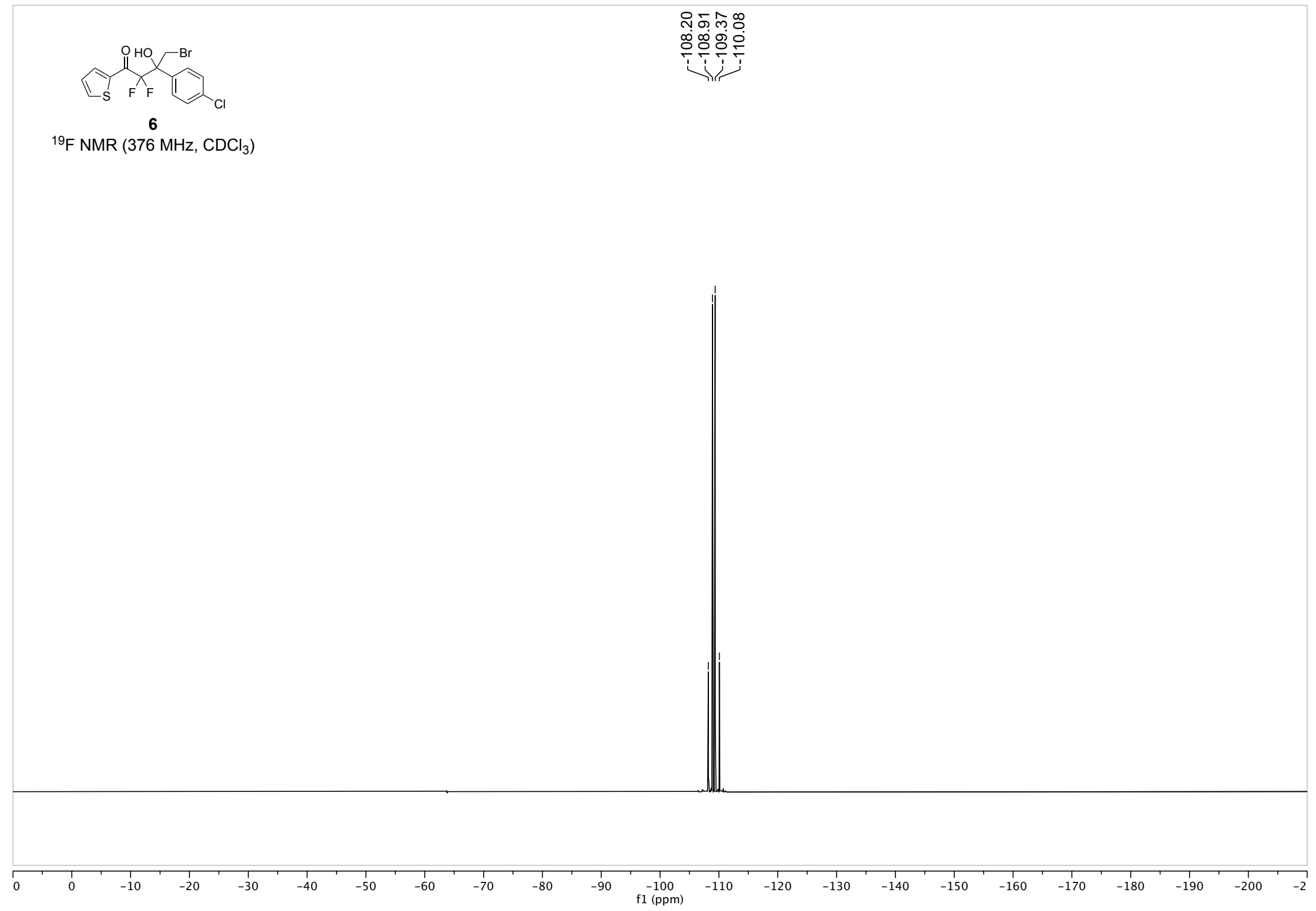

ฟे 


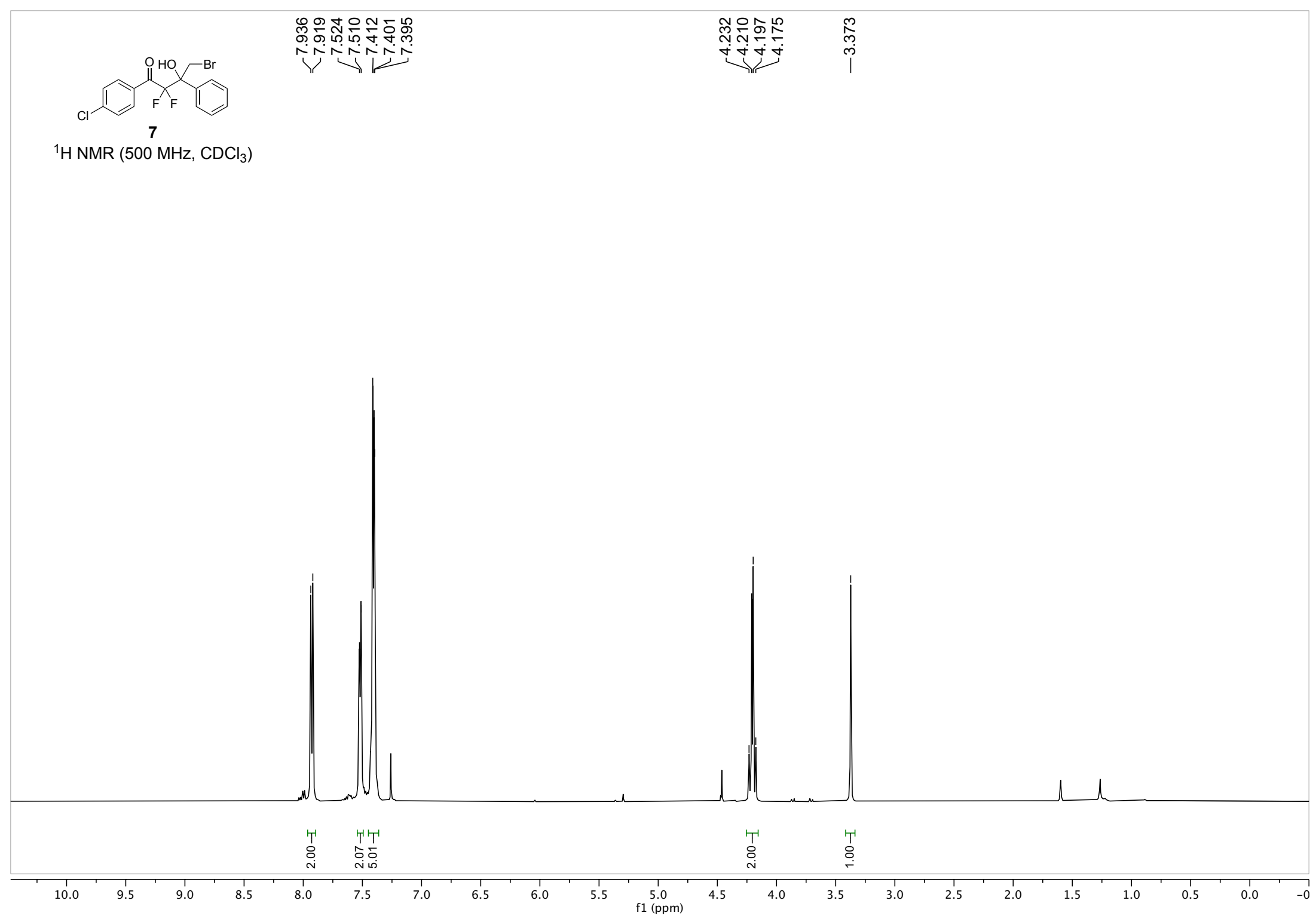




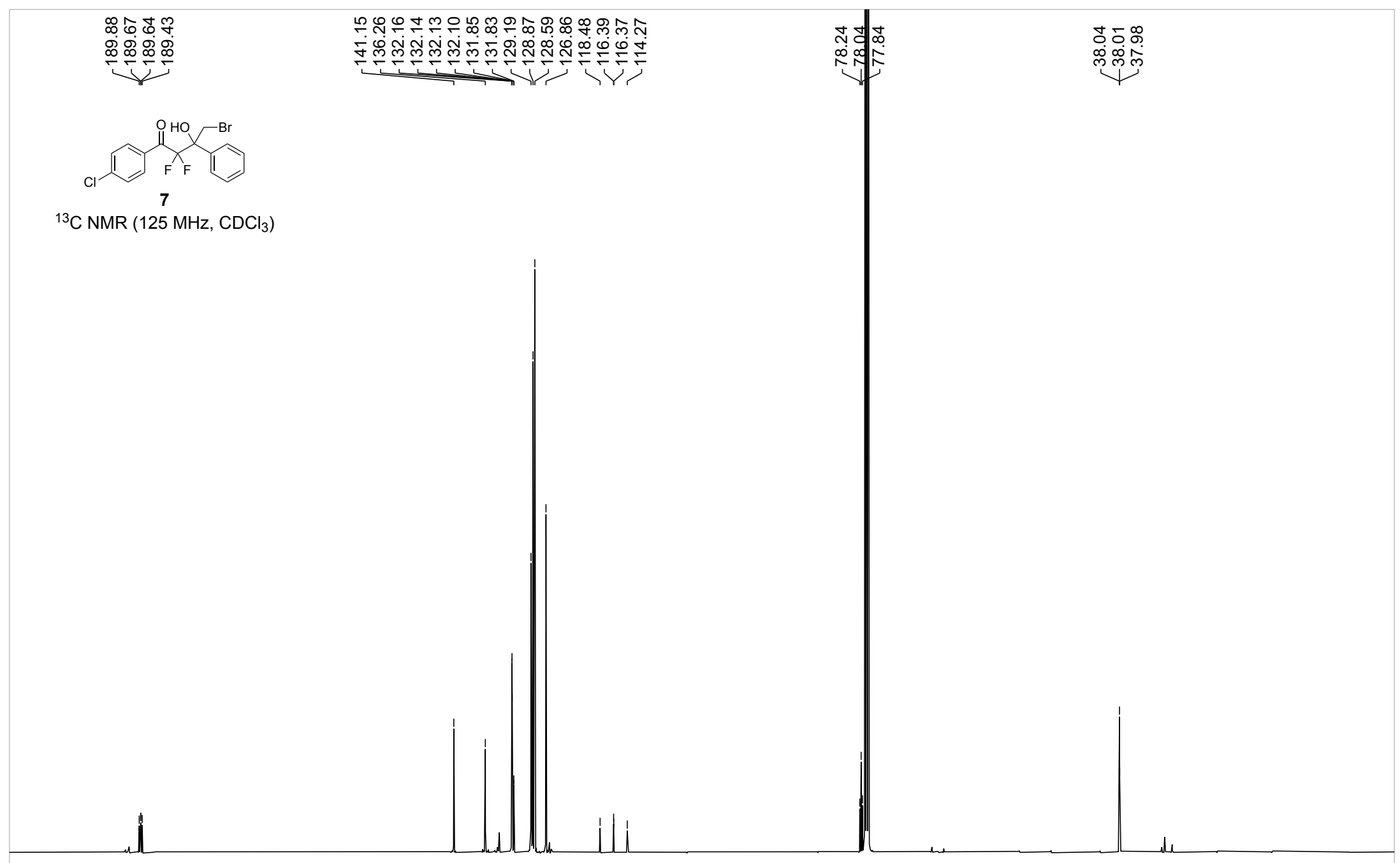

$\bar{n}$

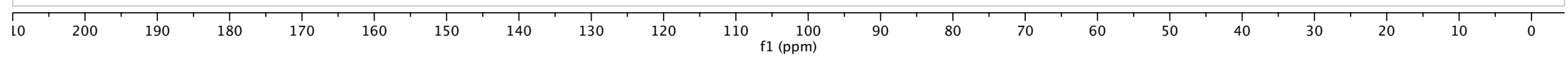




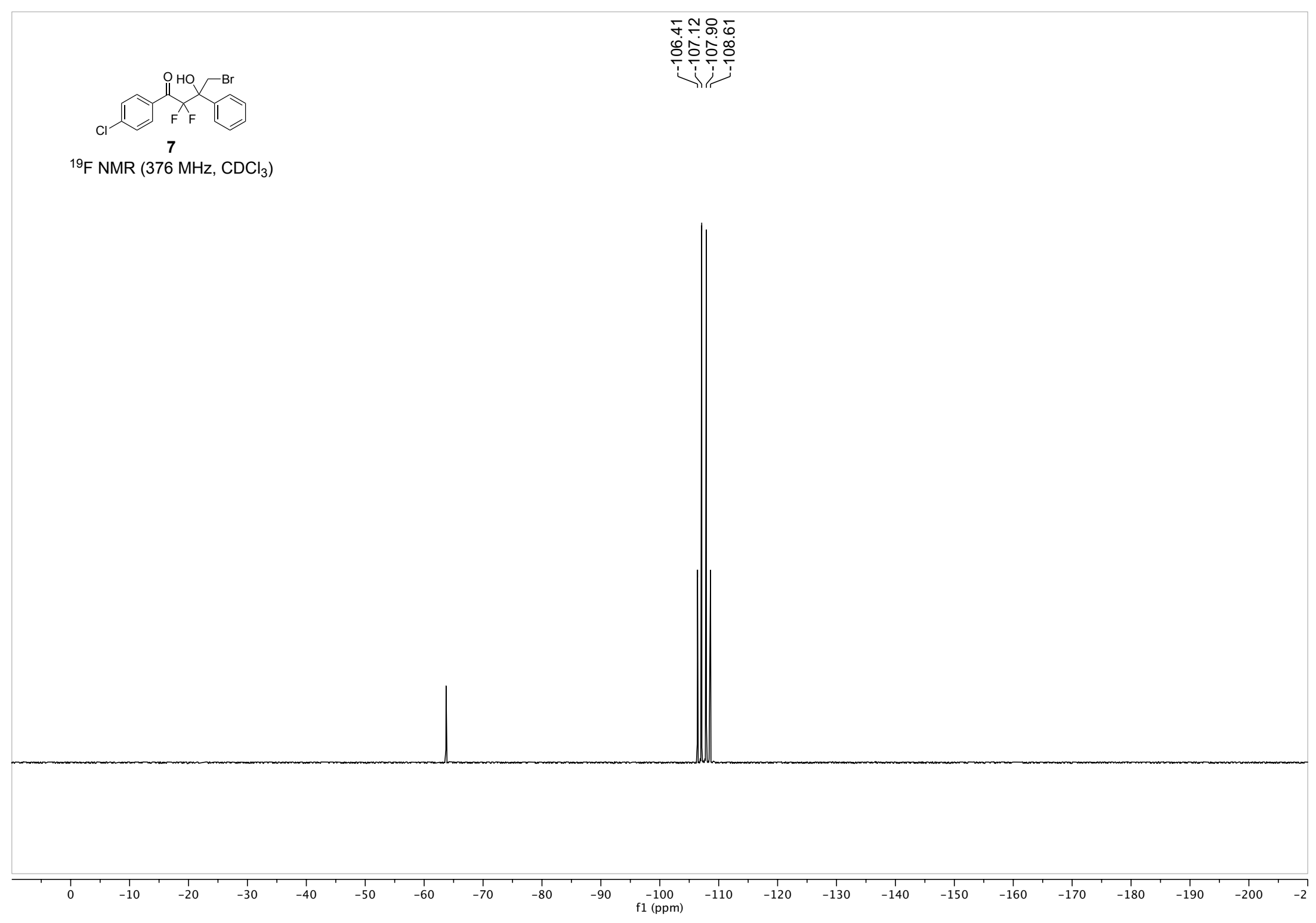




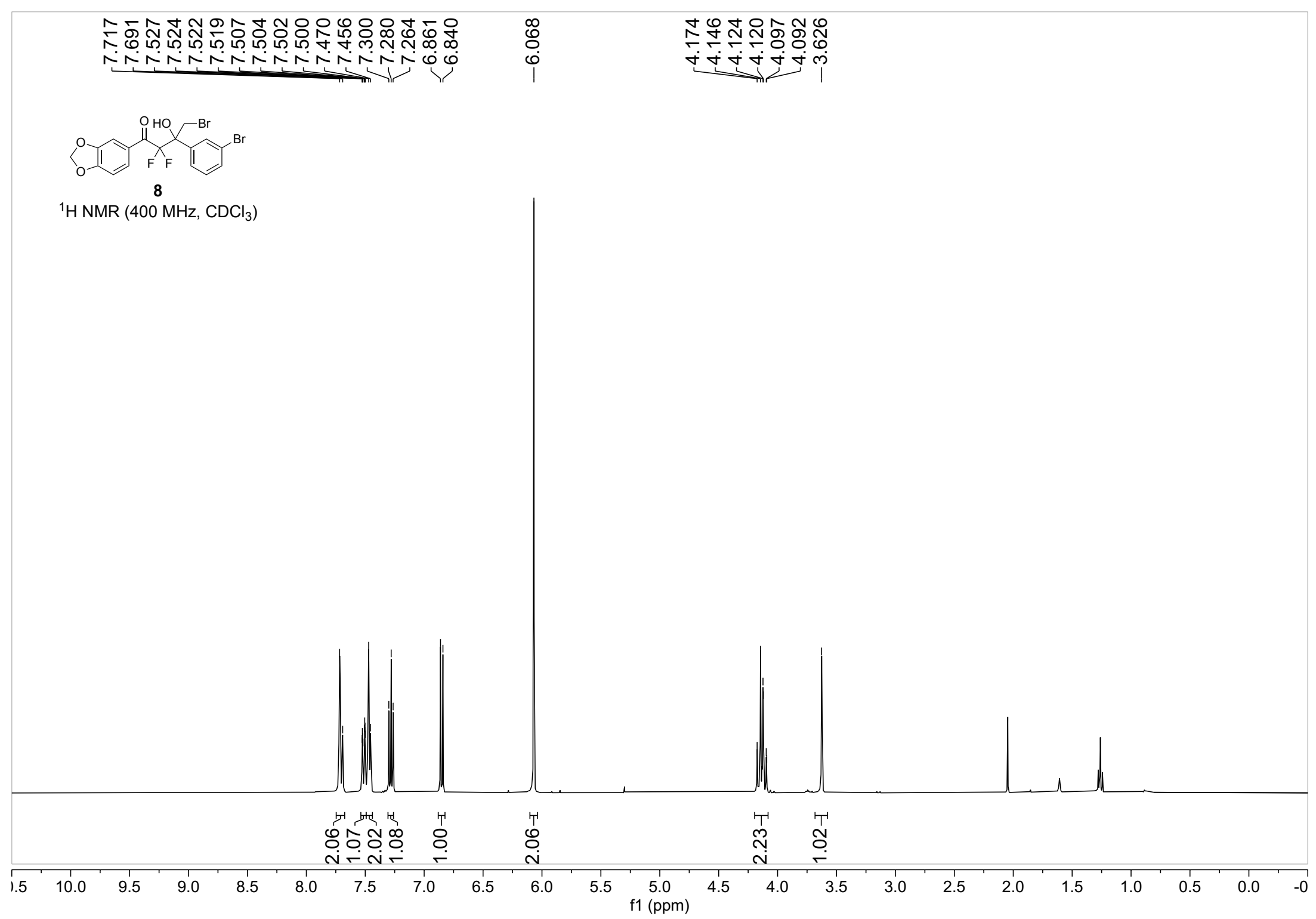




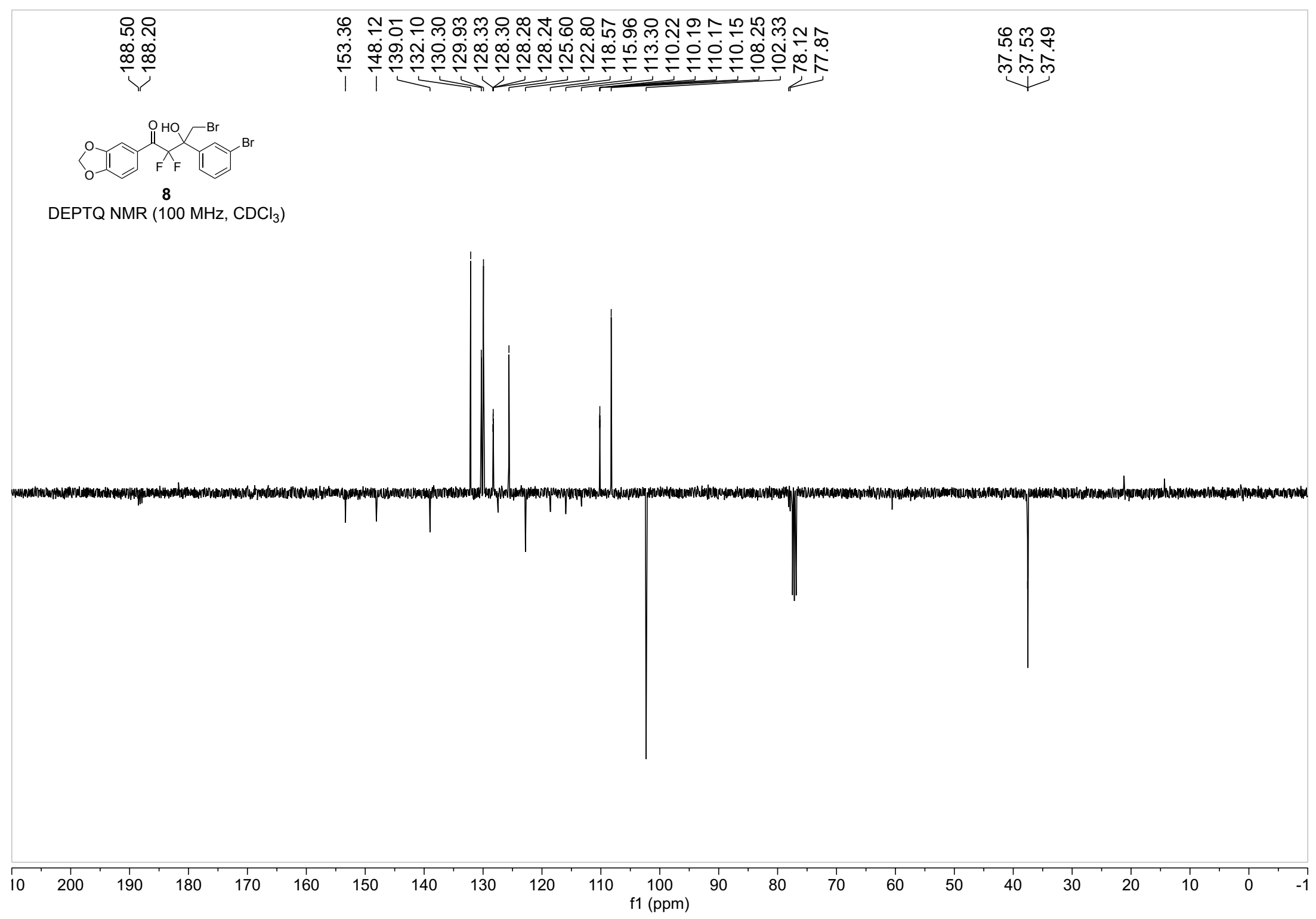

D 


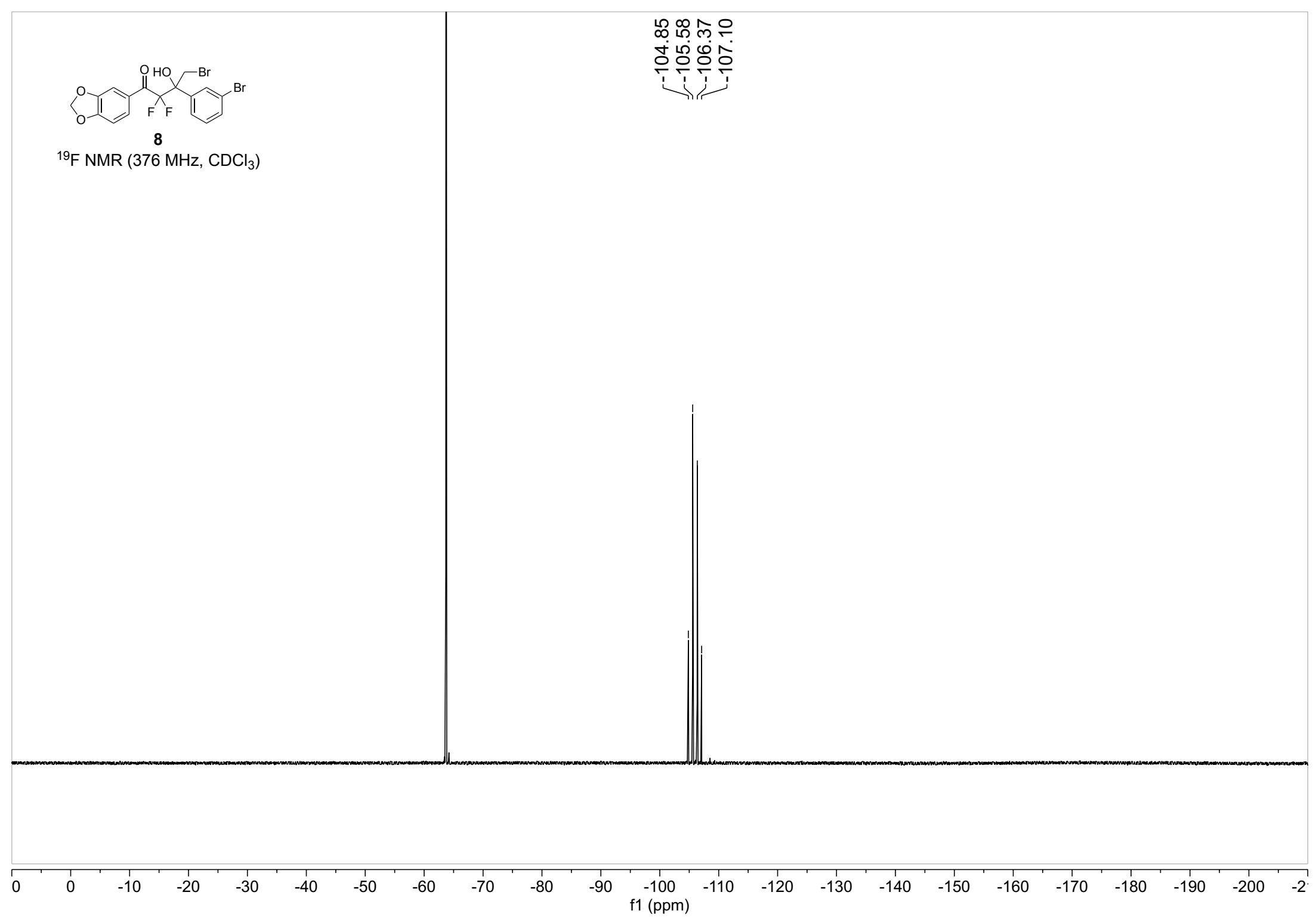




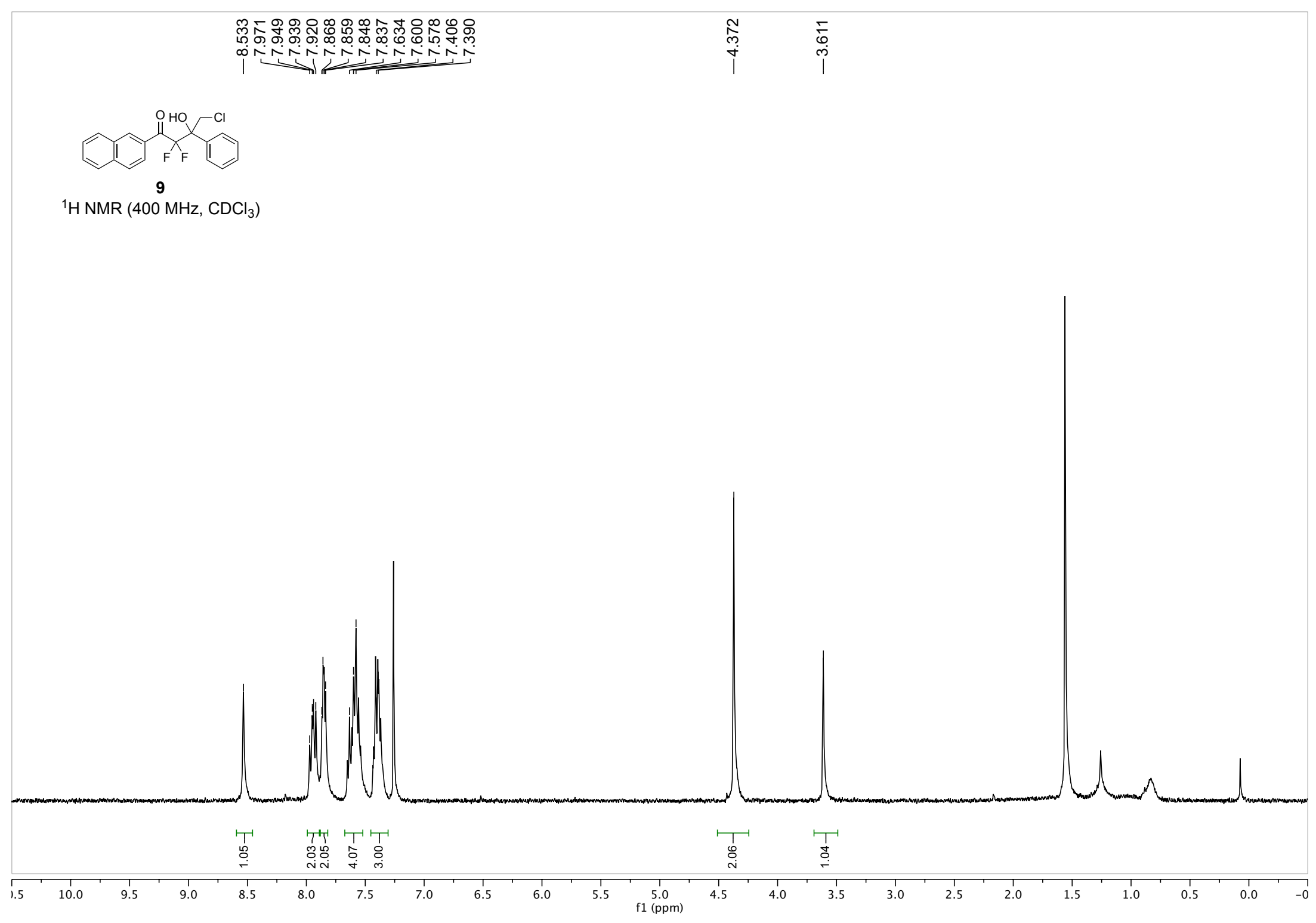




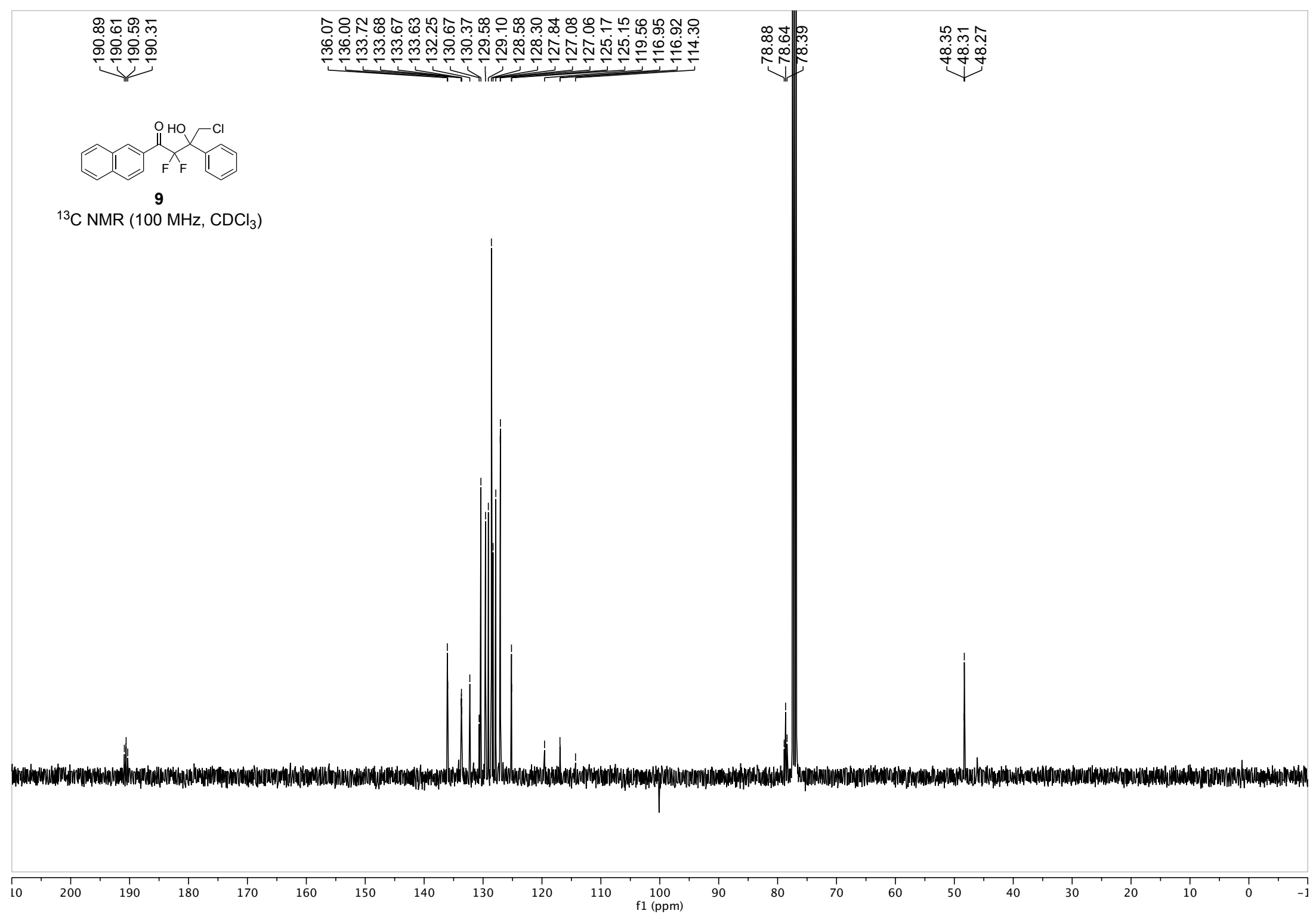




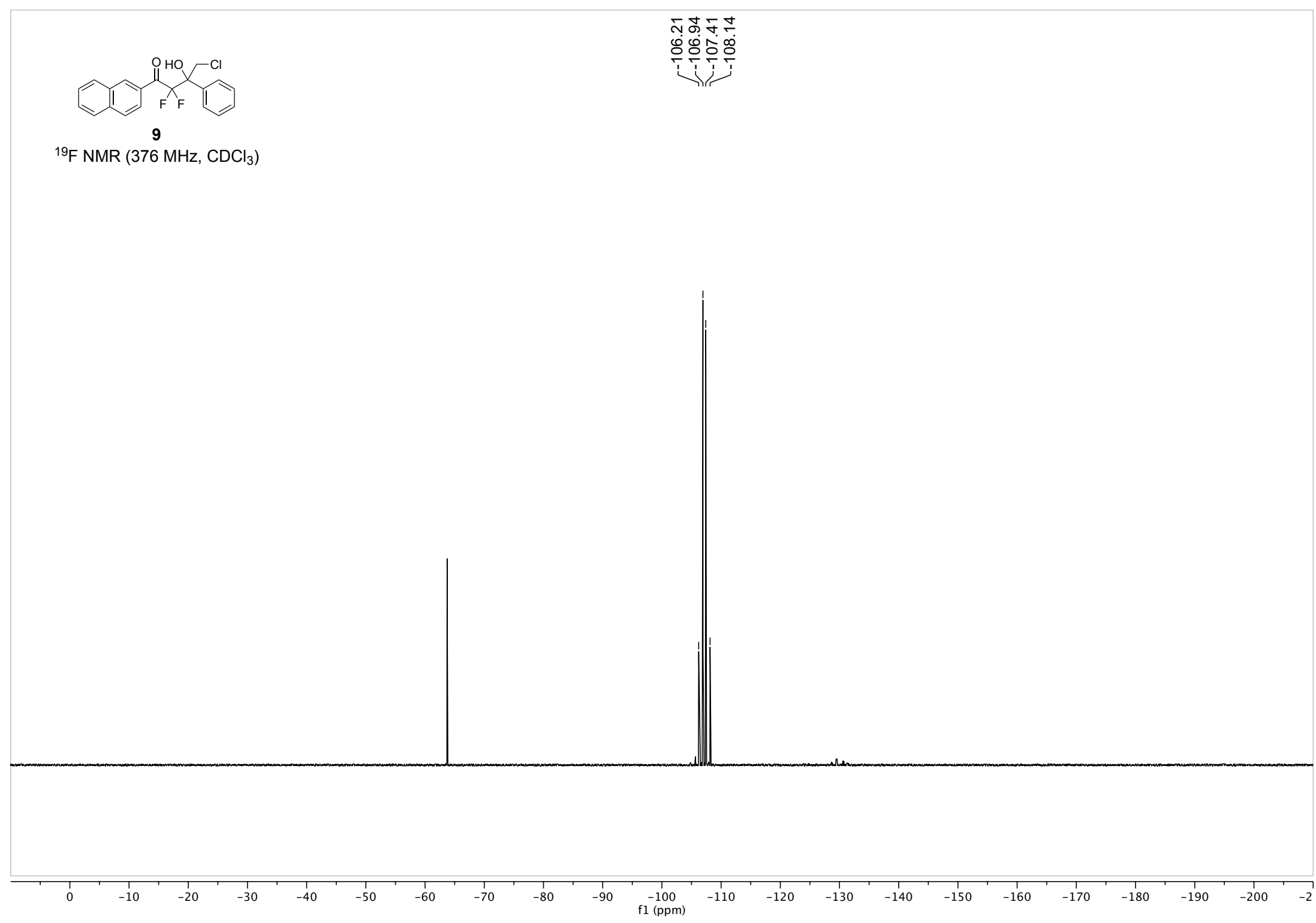




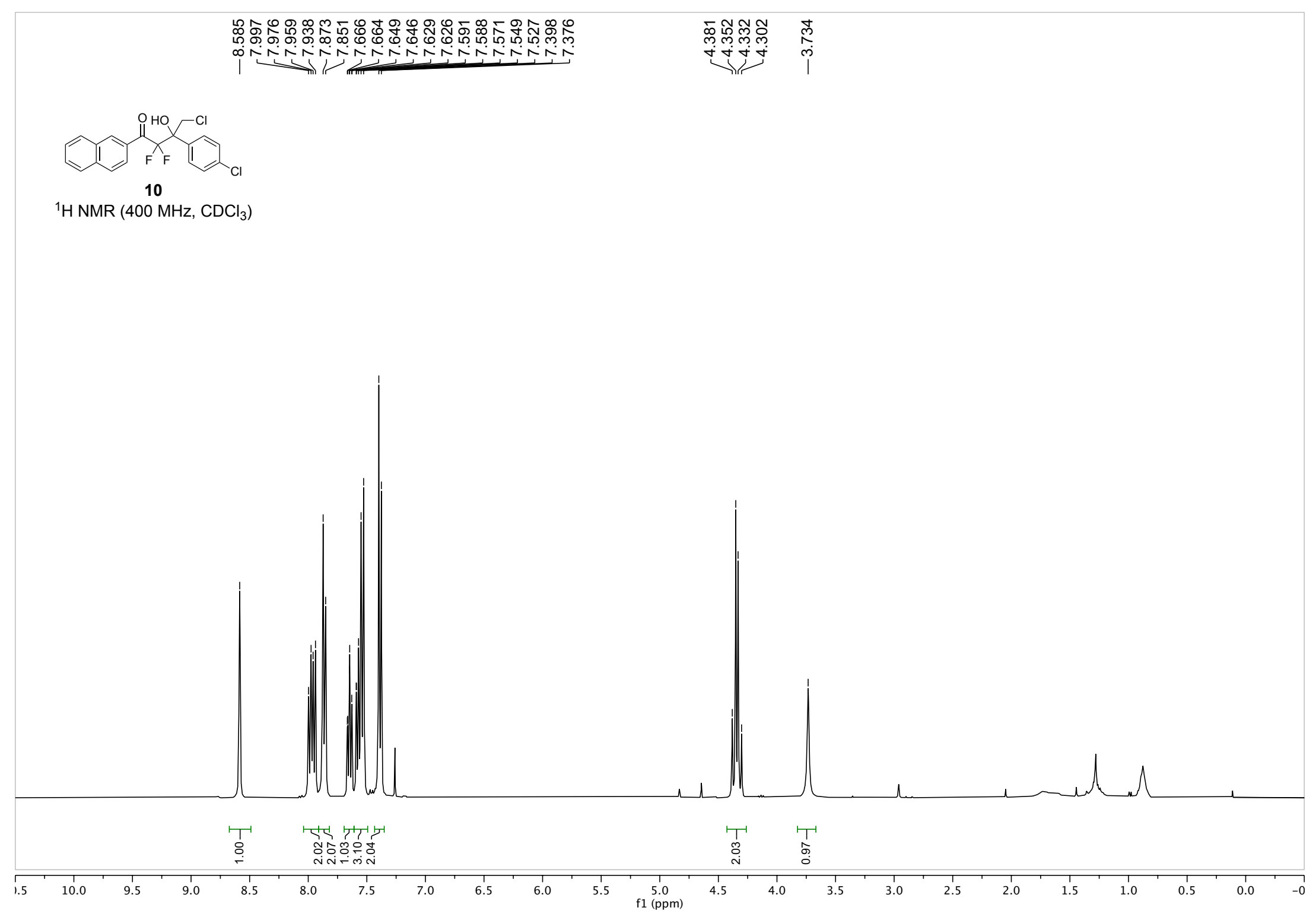




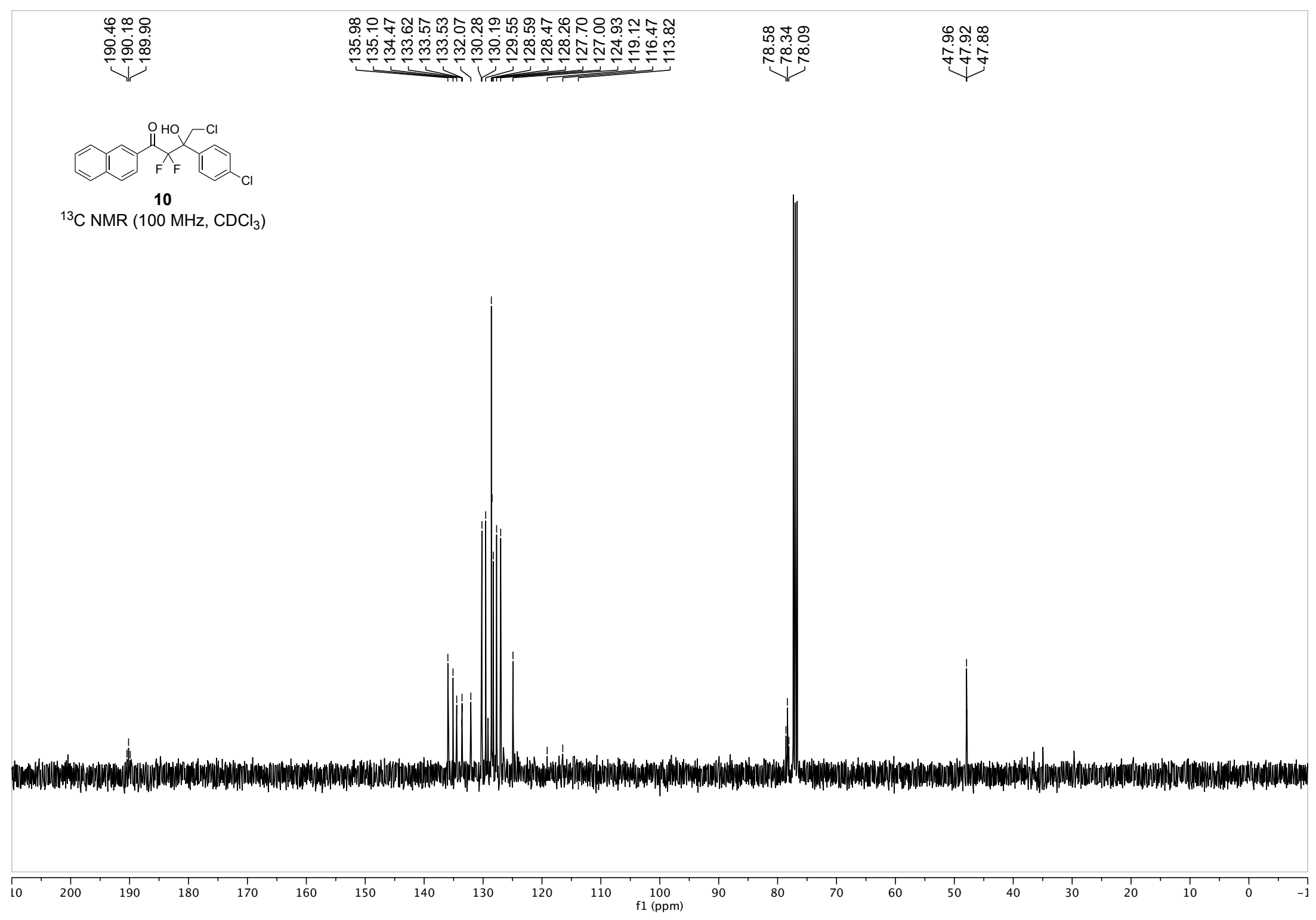


${ }^{19} \mathrm{~F} \mathrm{NMR} \mathrm{(376} \mathrm{MHz,} \mathrm{CDCl}_{3}$ )

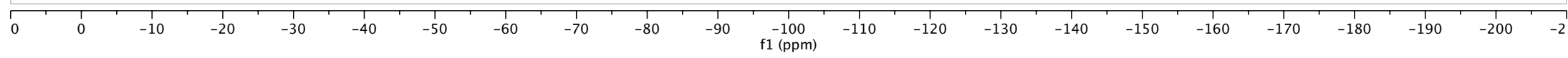




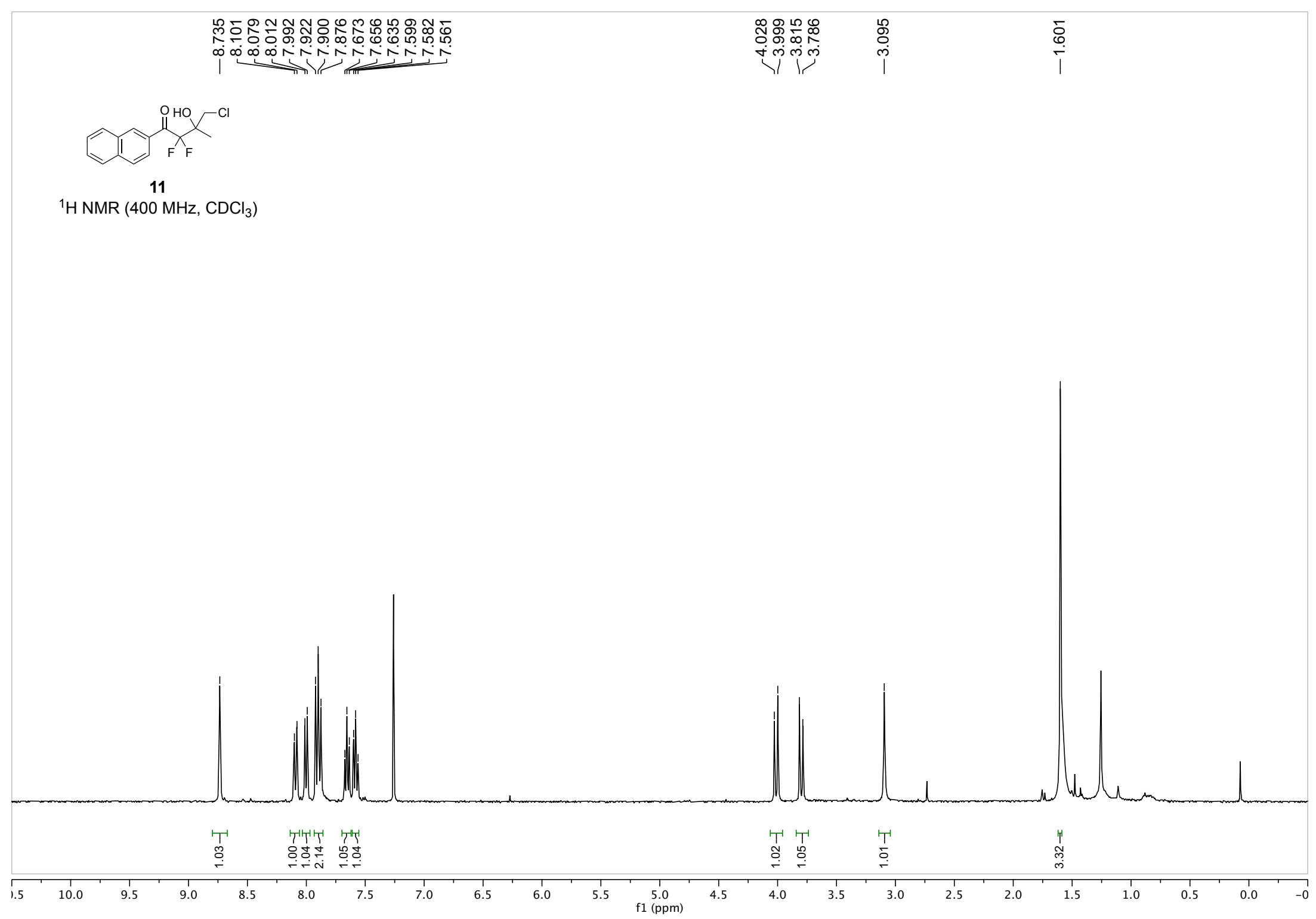




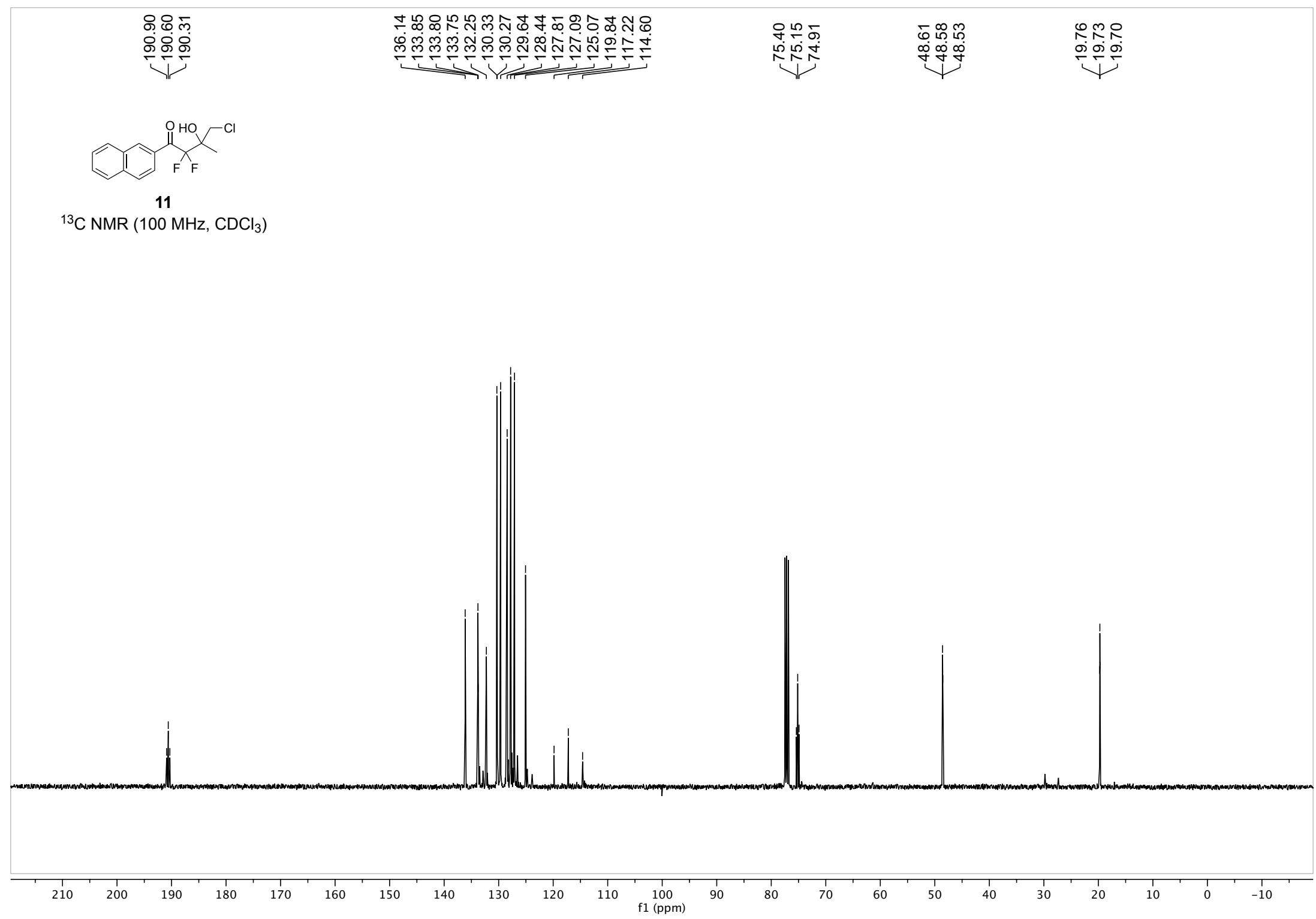




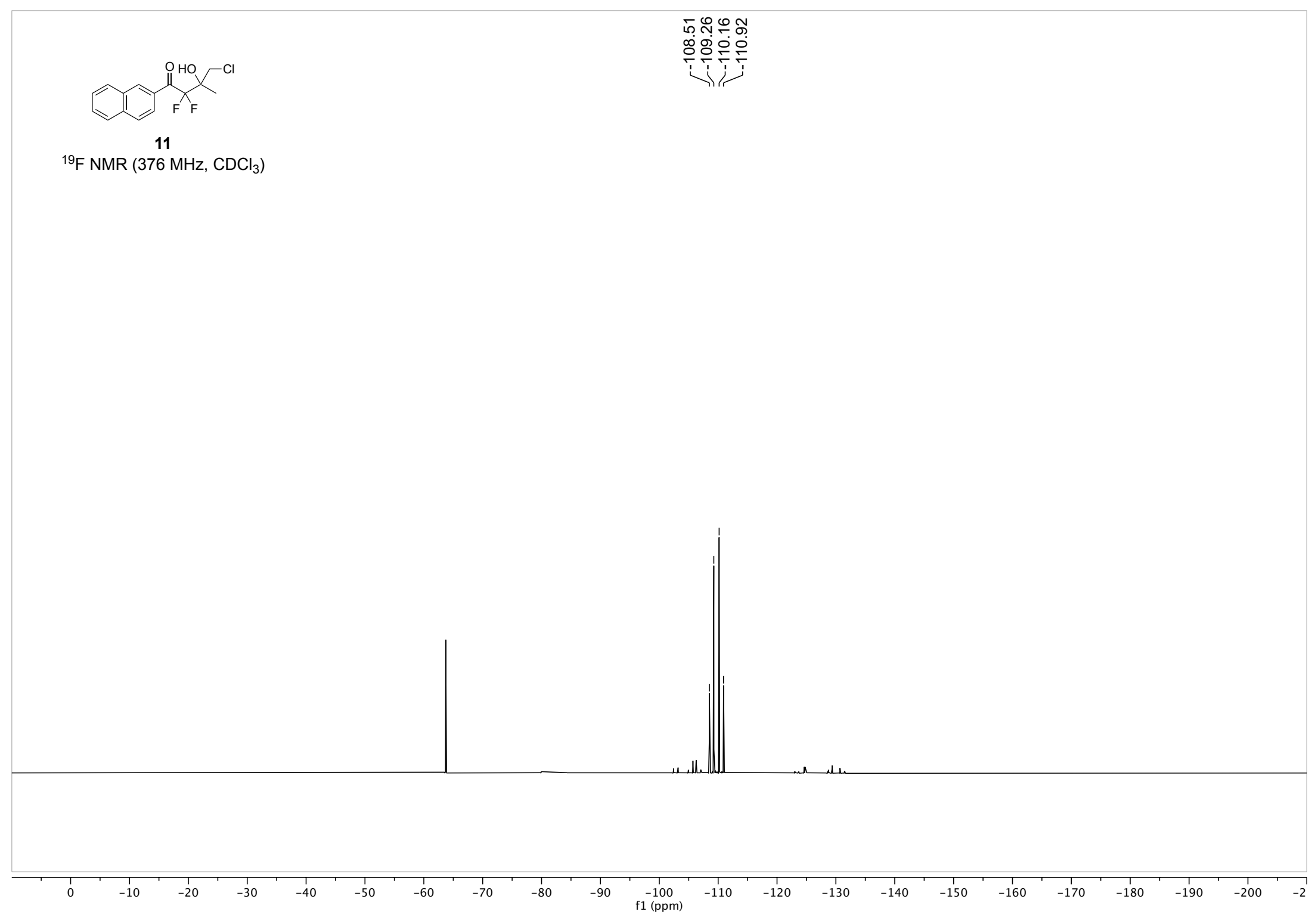




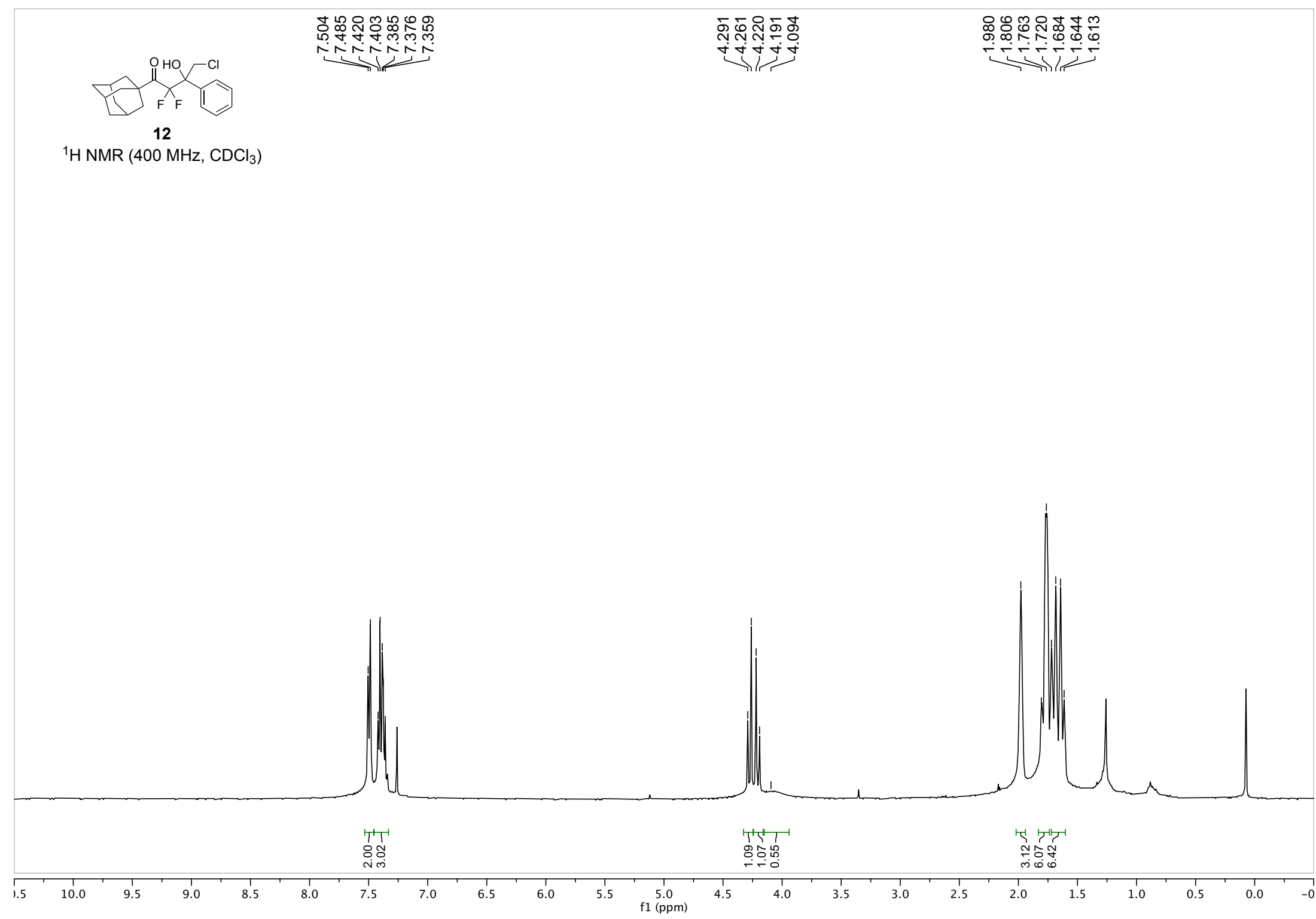




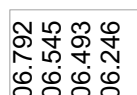

ํㅠㅇㅠ

郘

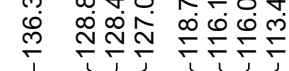

| प।
조ํำำ

ำ

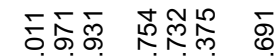

告年

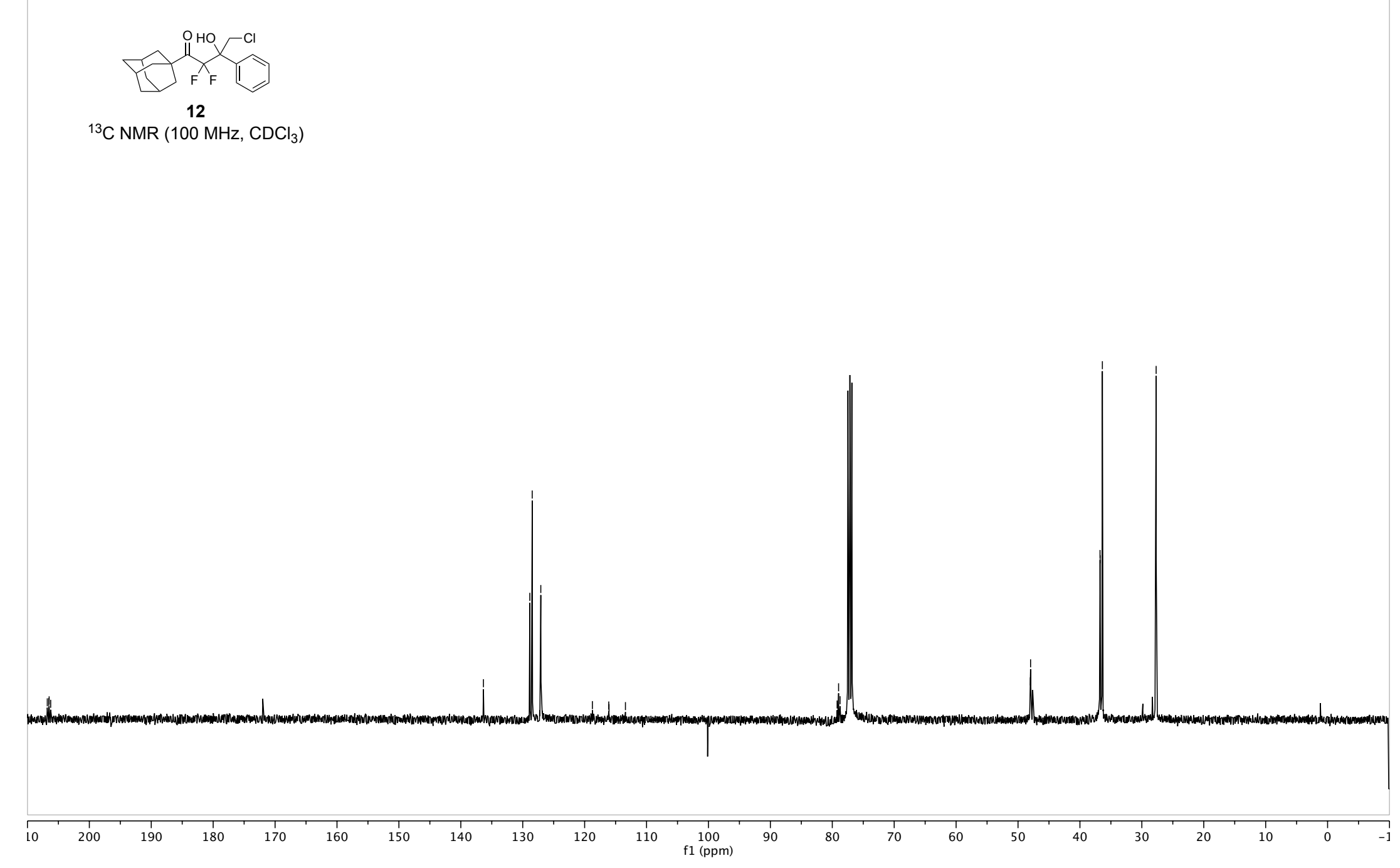




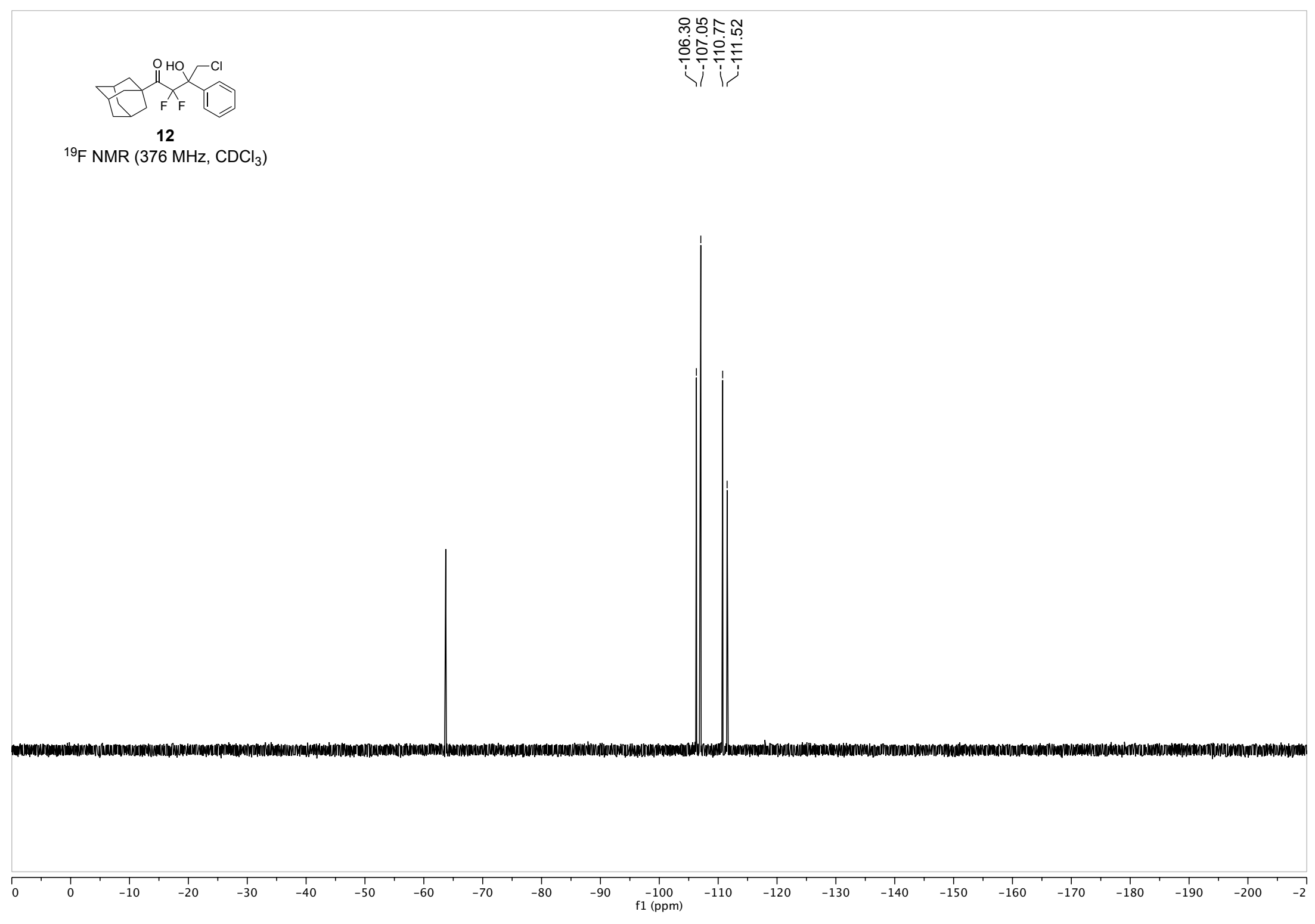




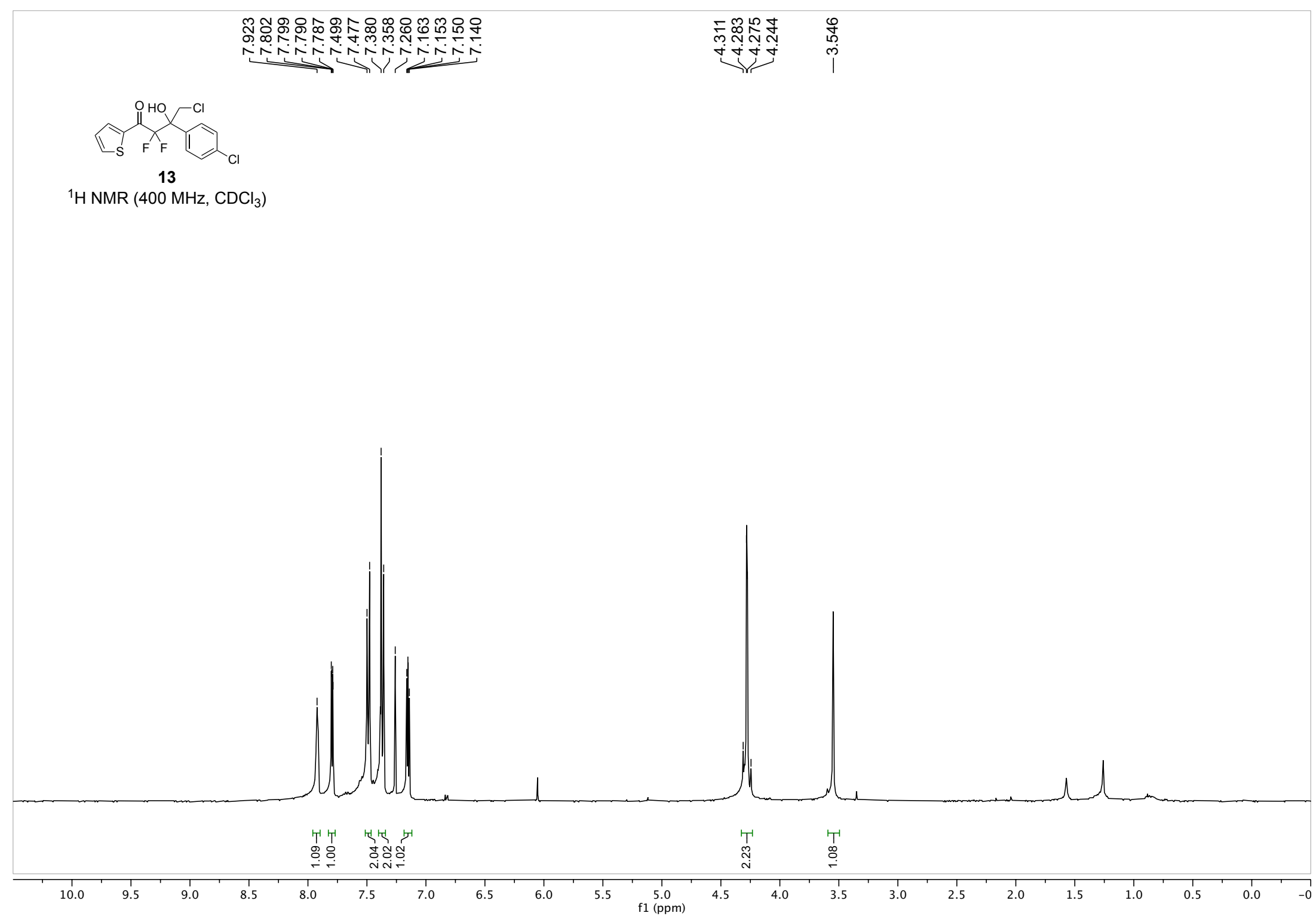




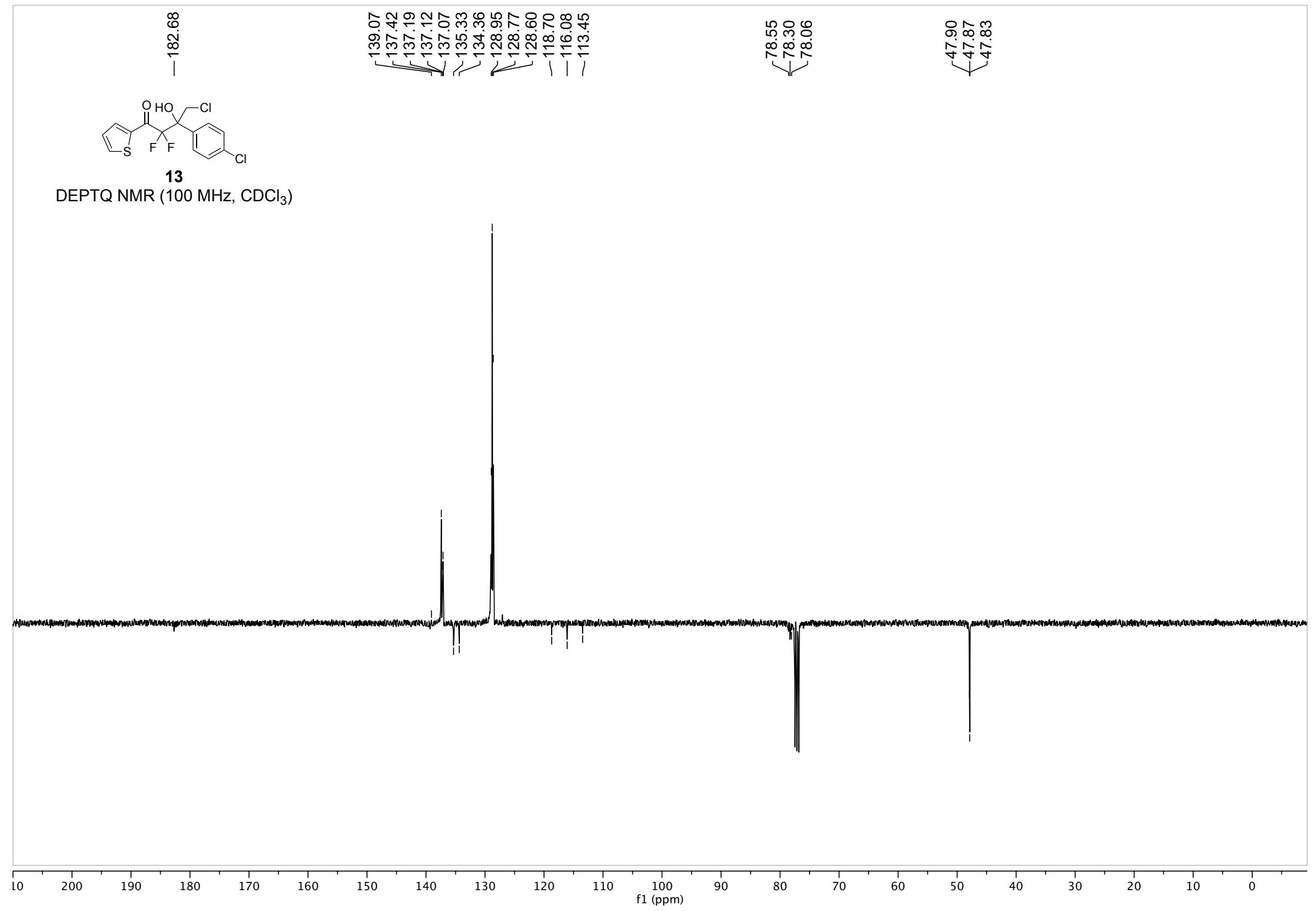



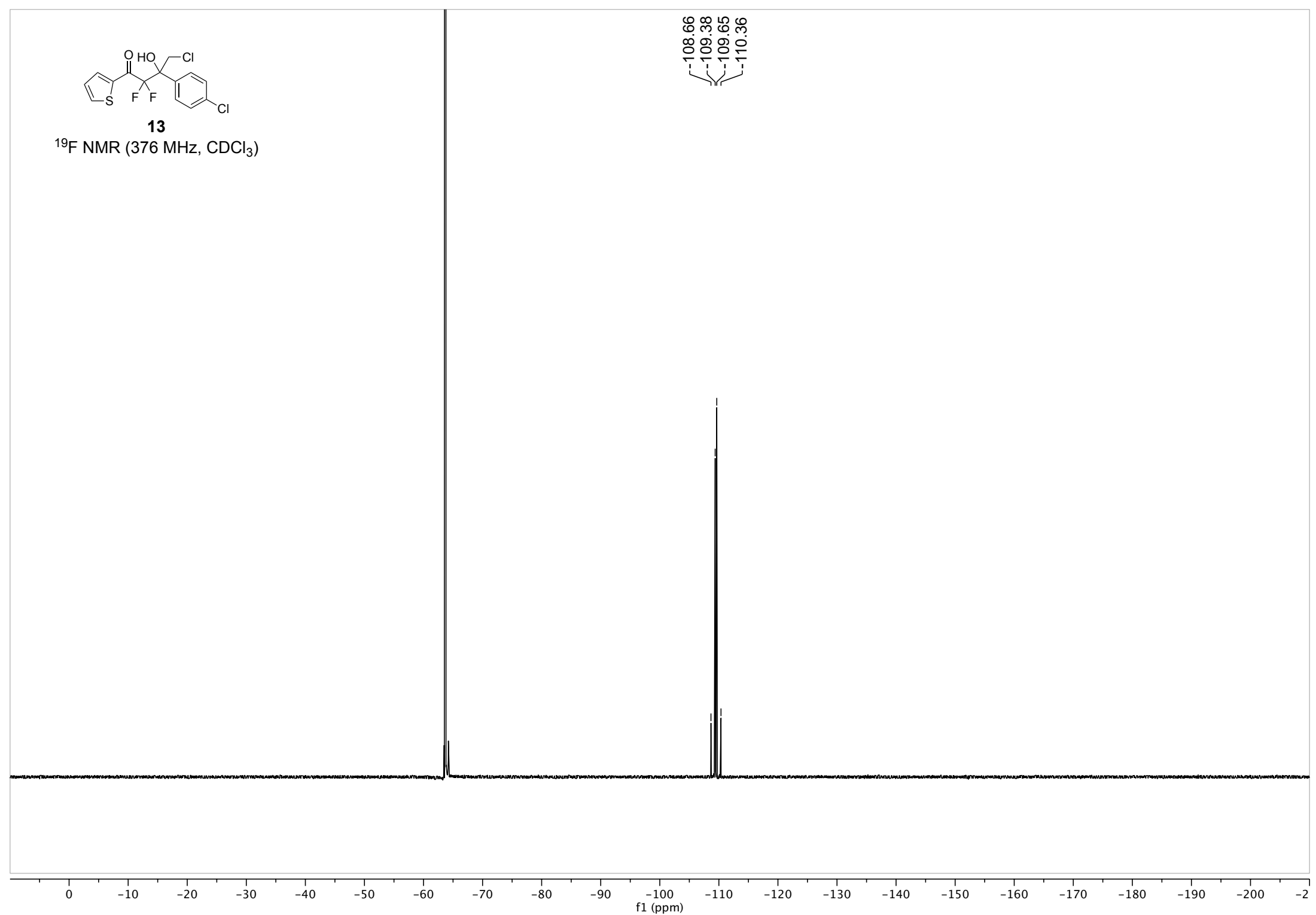


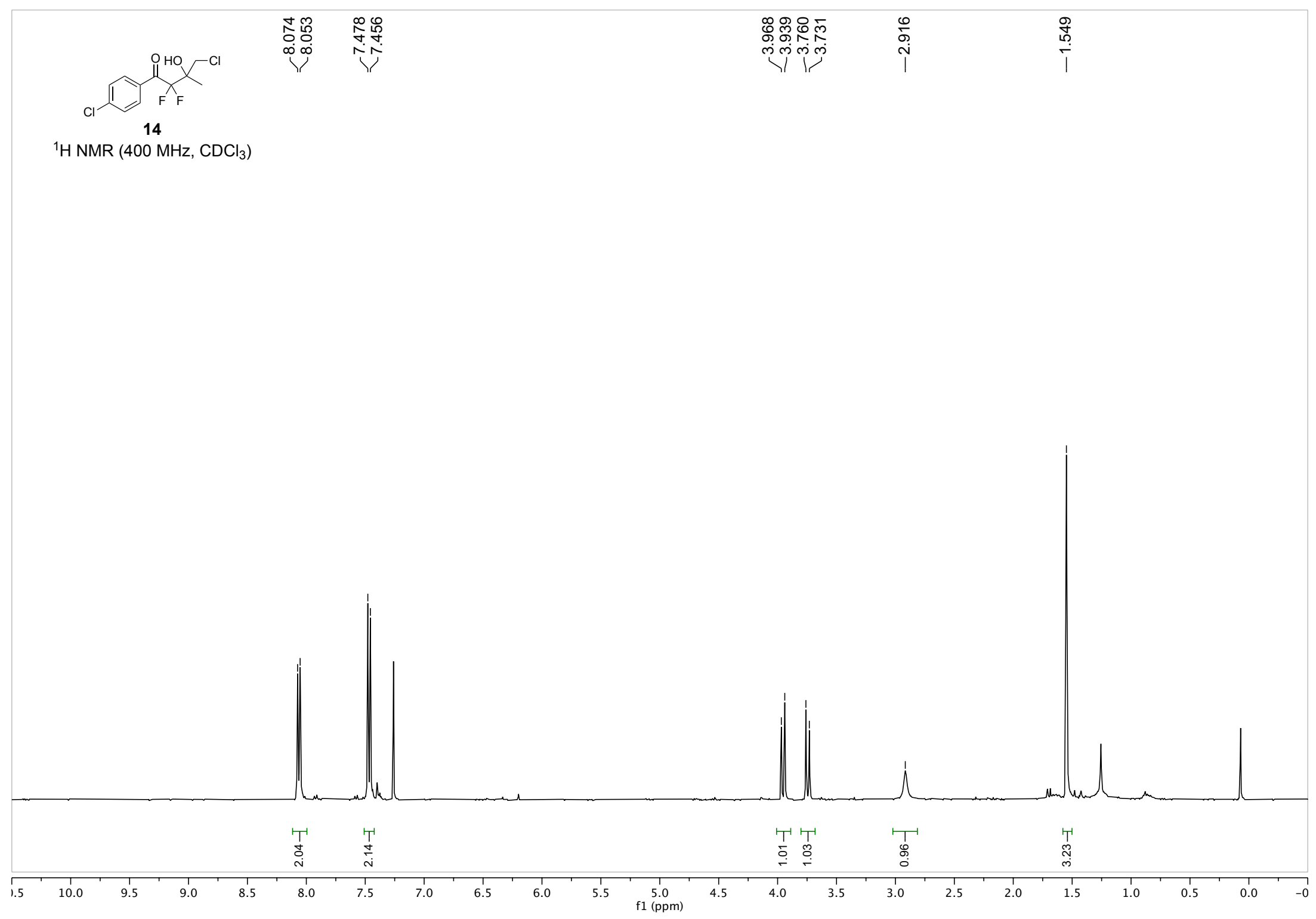




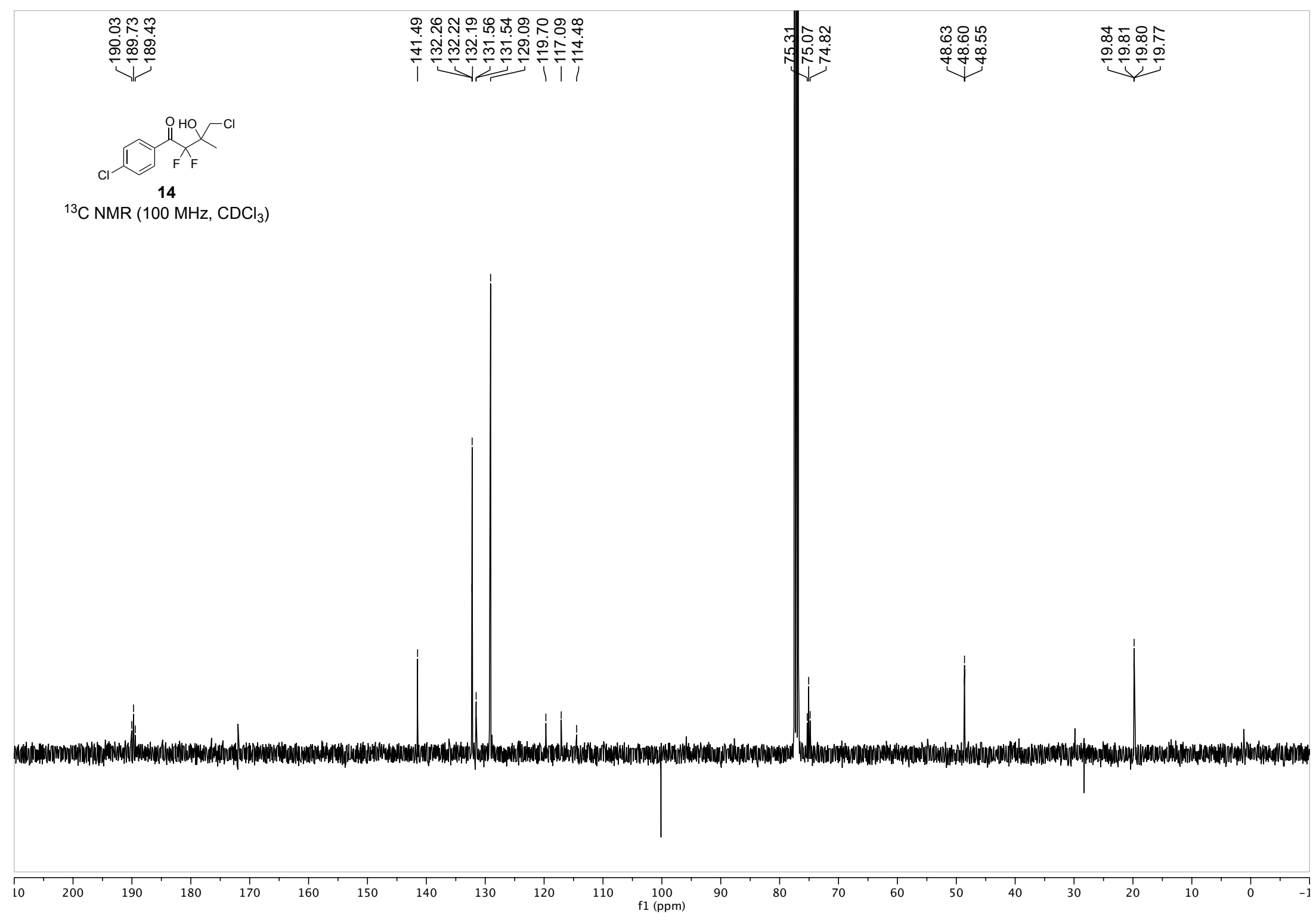



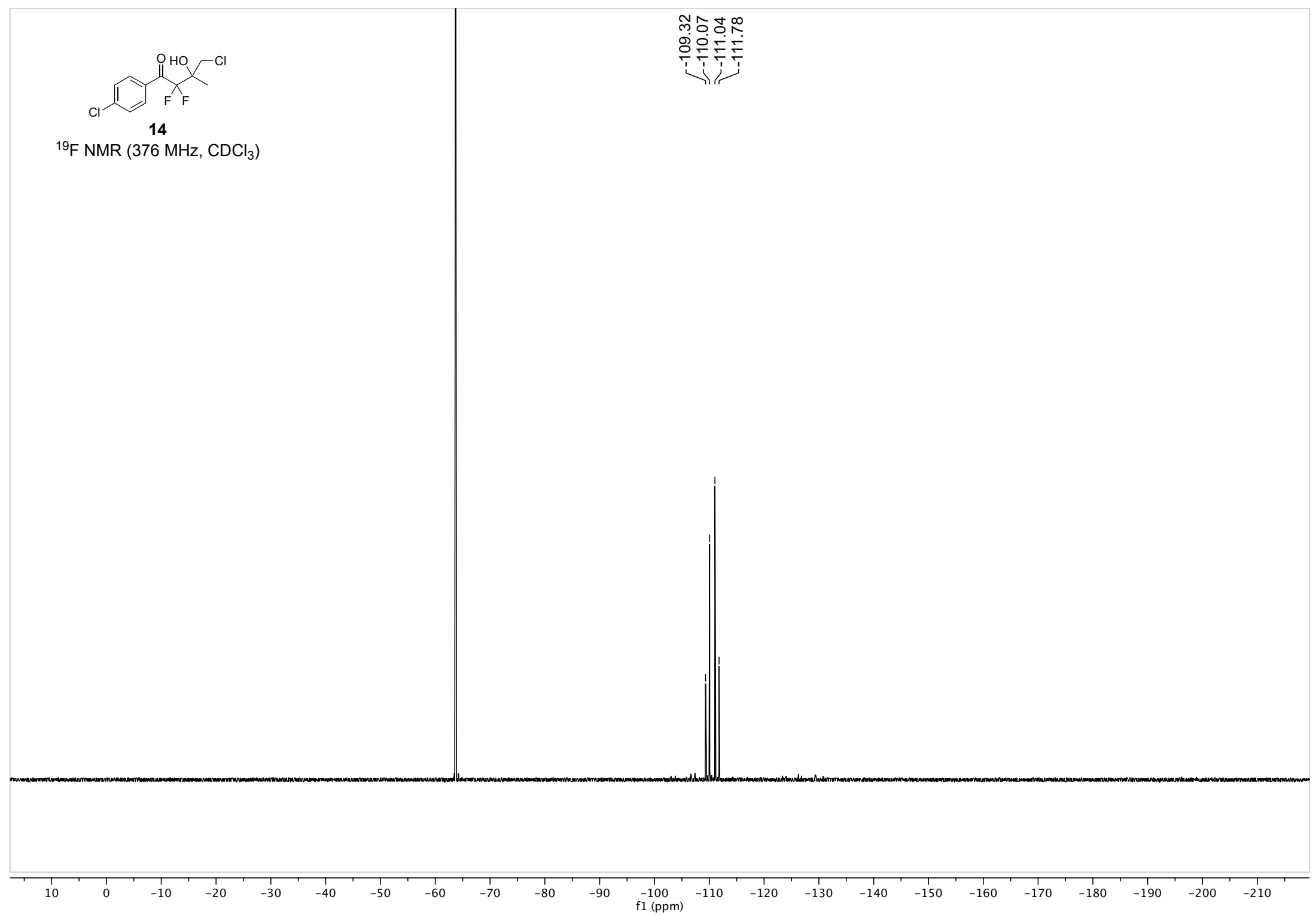


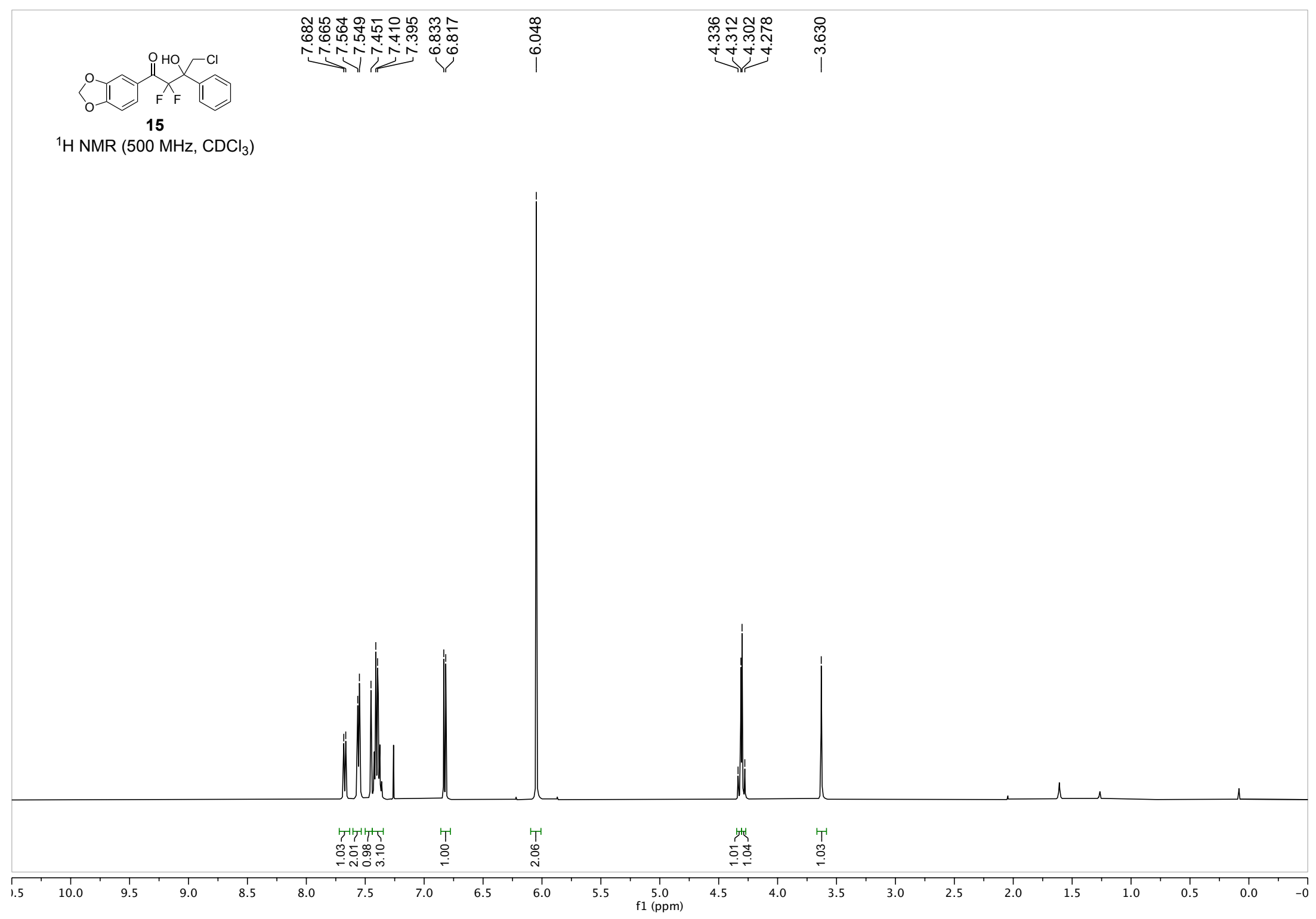




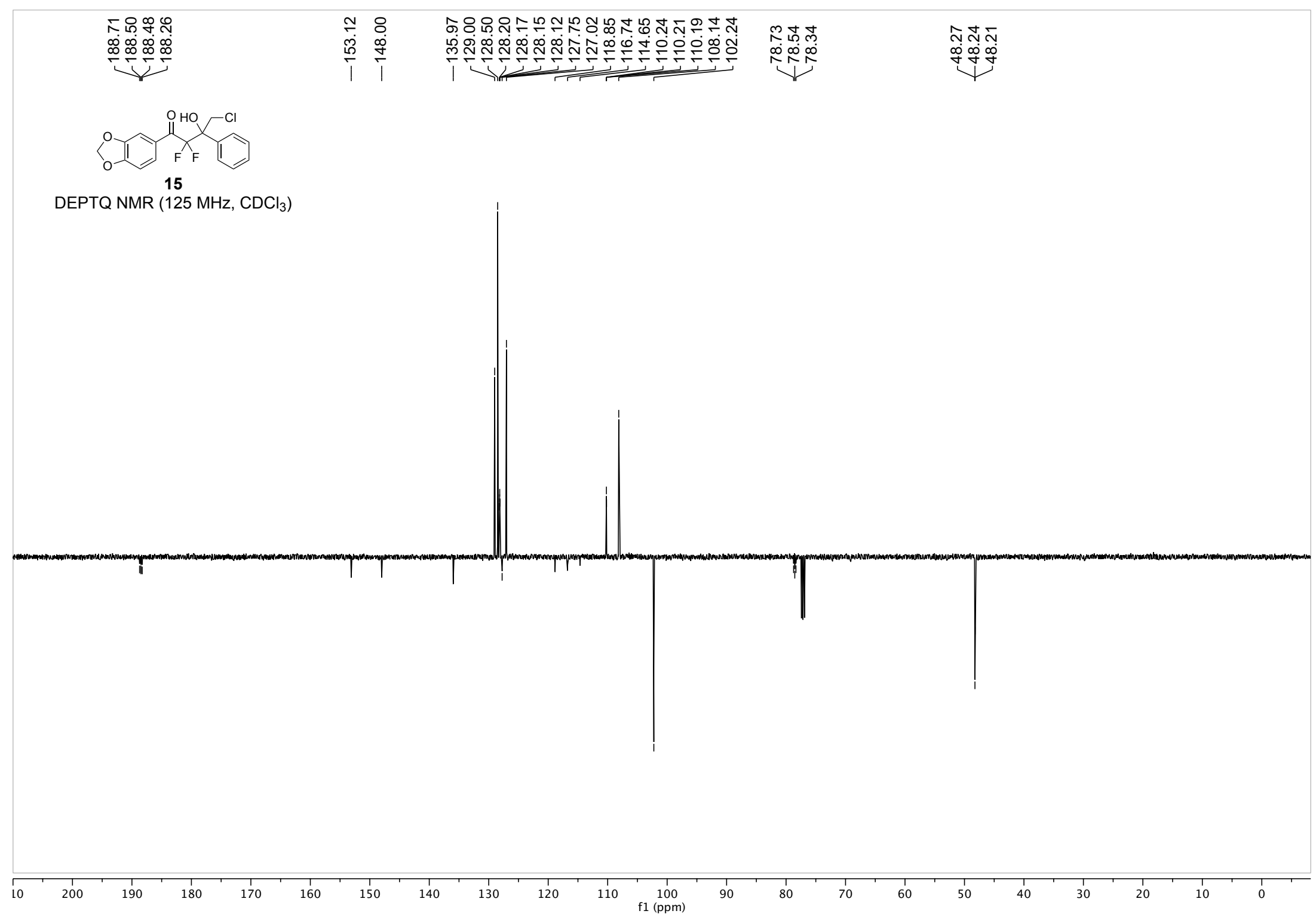




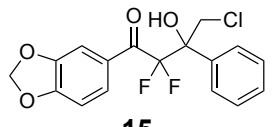

i

${ }^{19} \mathrm{~F} \mathrm{NMR}\left(376 \mathrm{MHz}, \mathrm{CDCl}_{3}\right)$

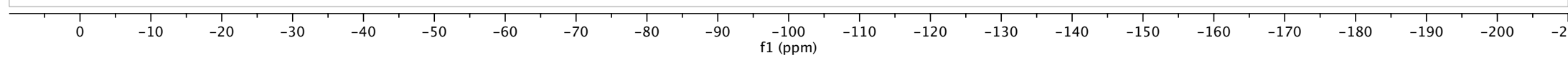




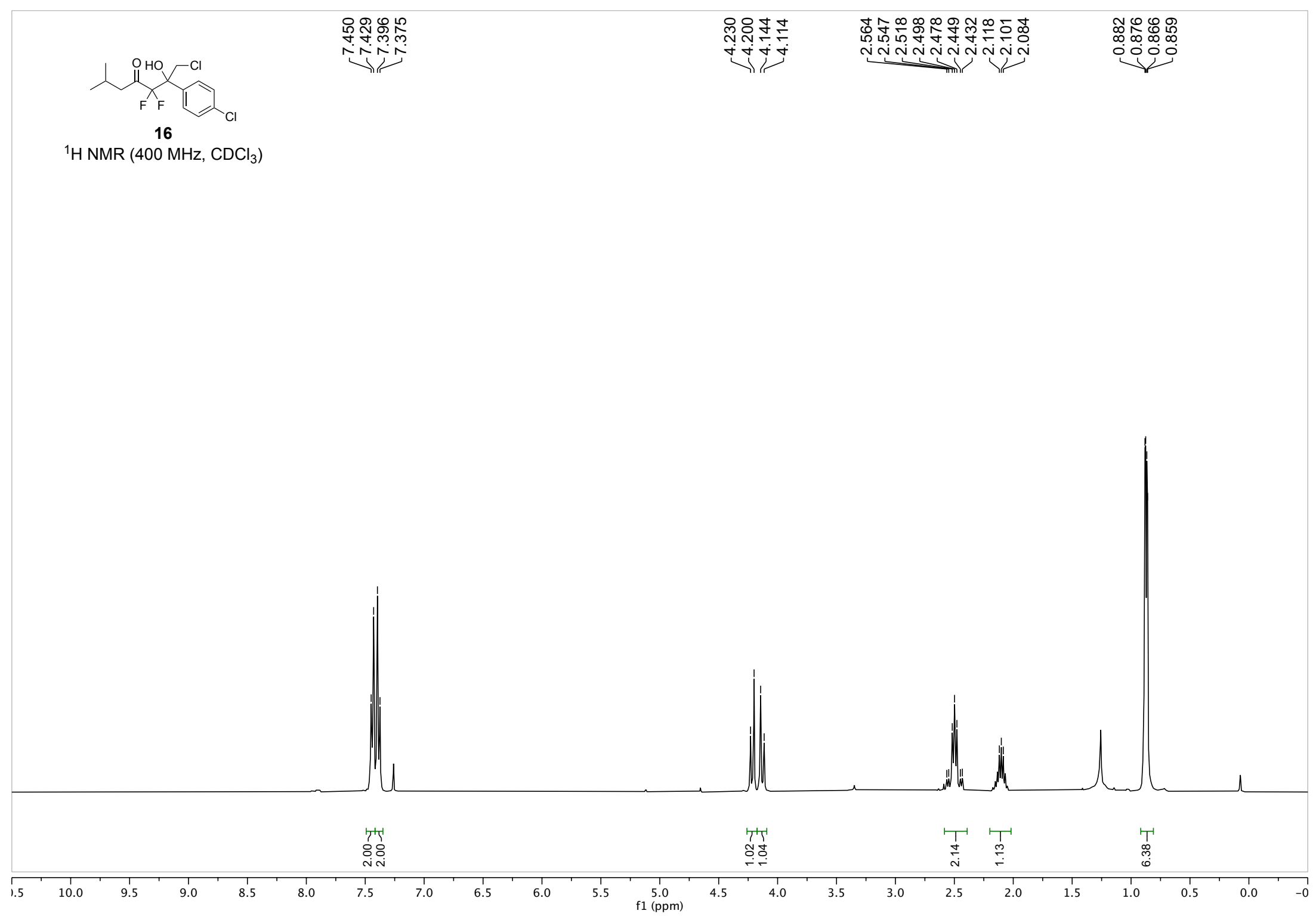




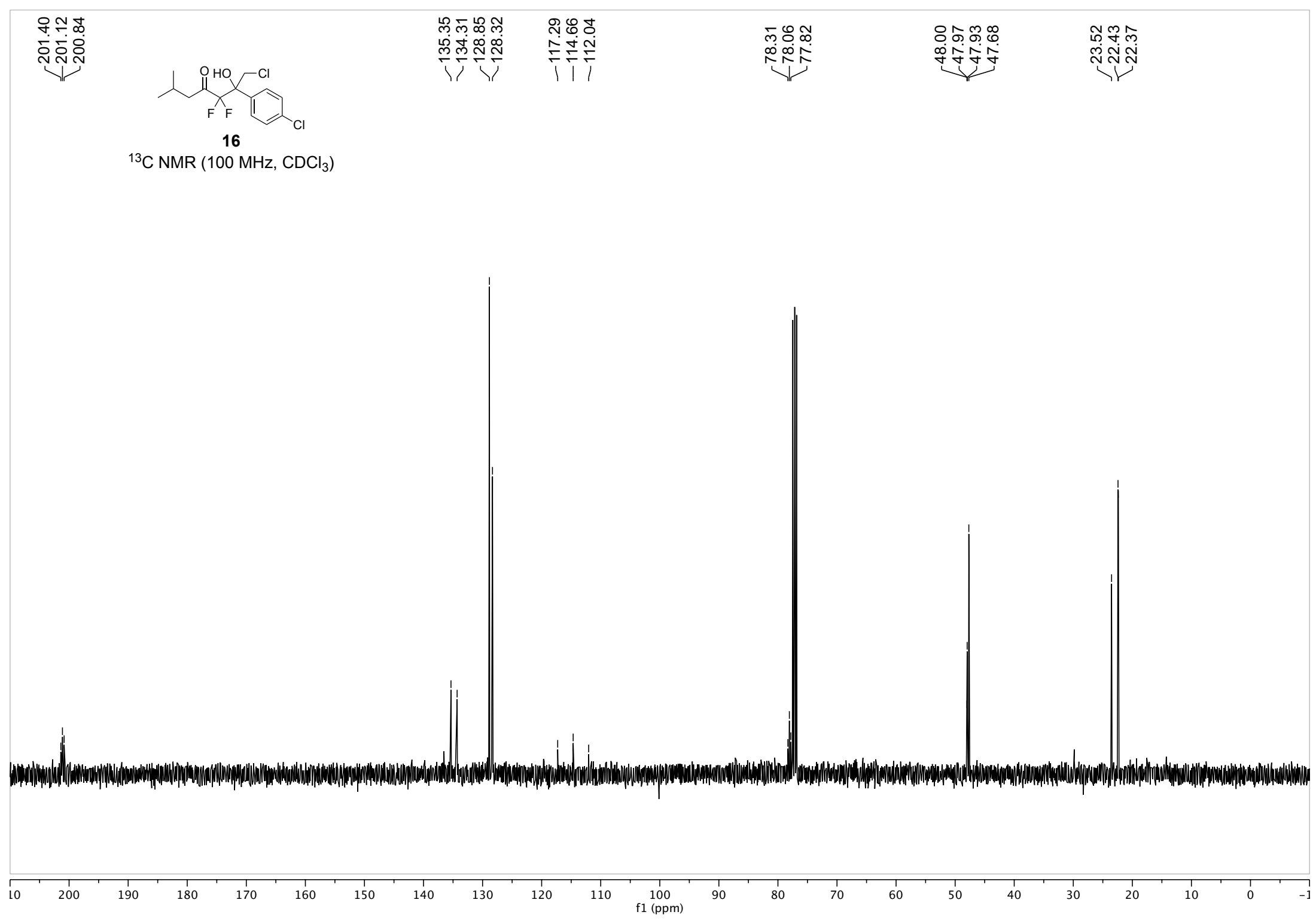




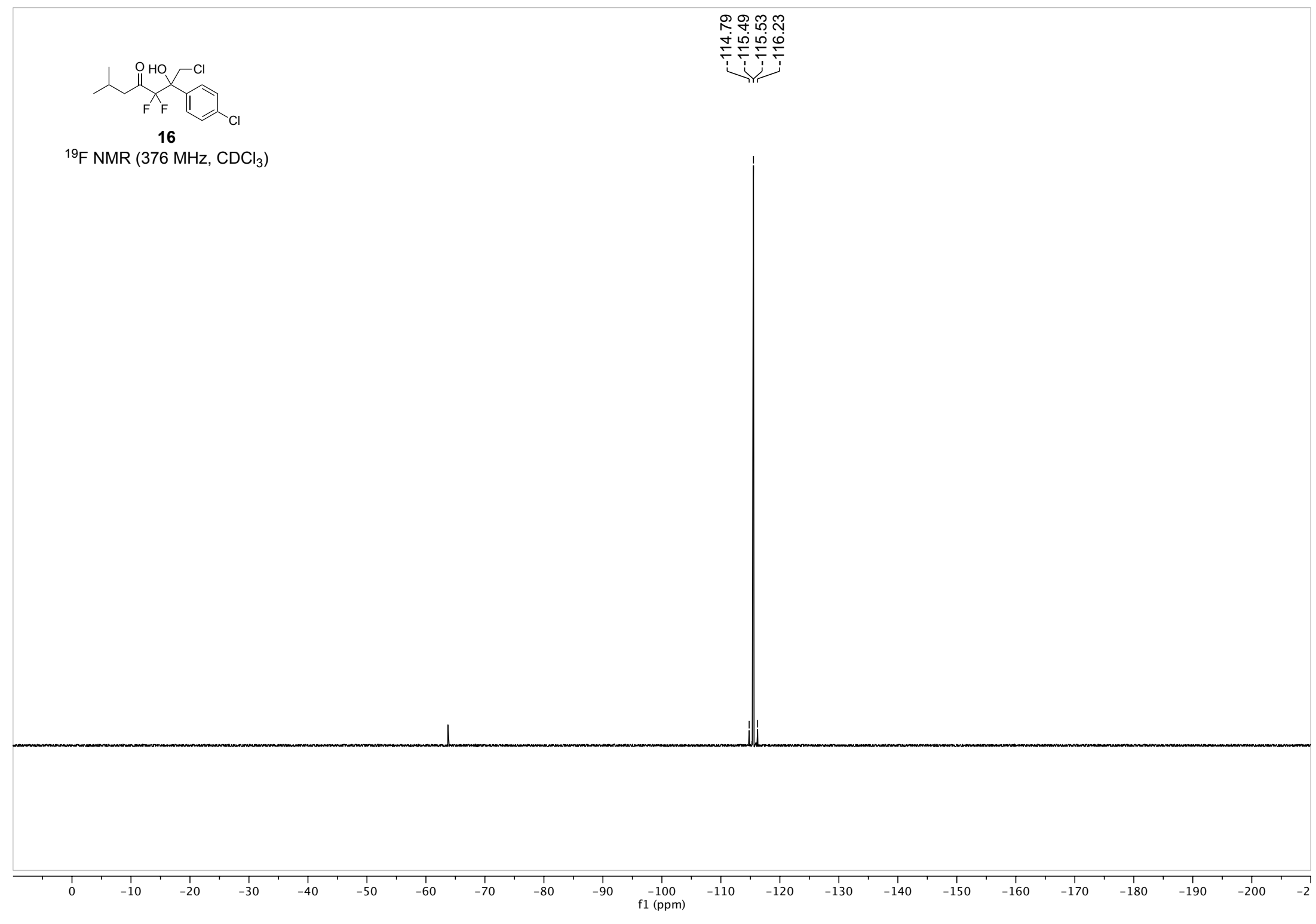




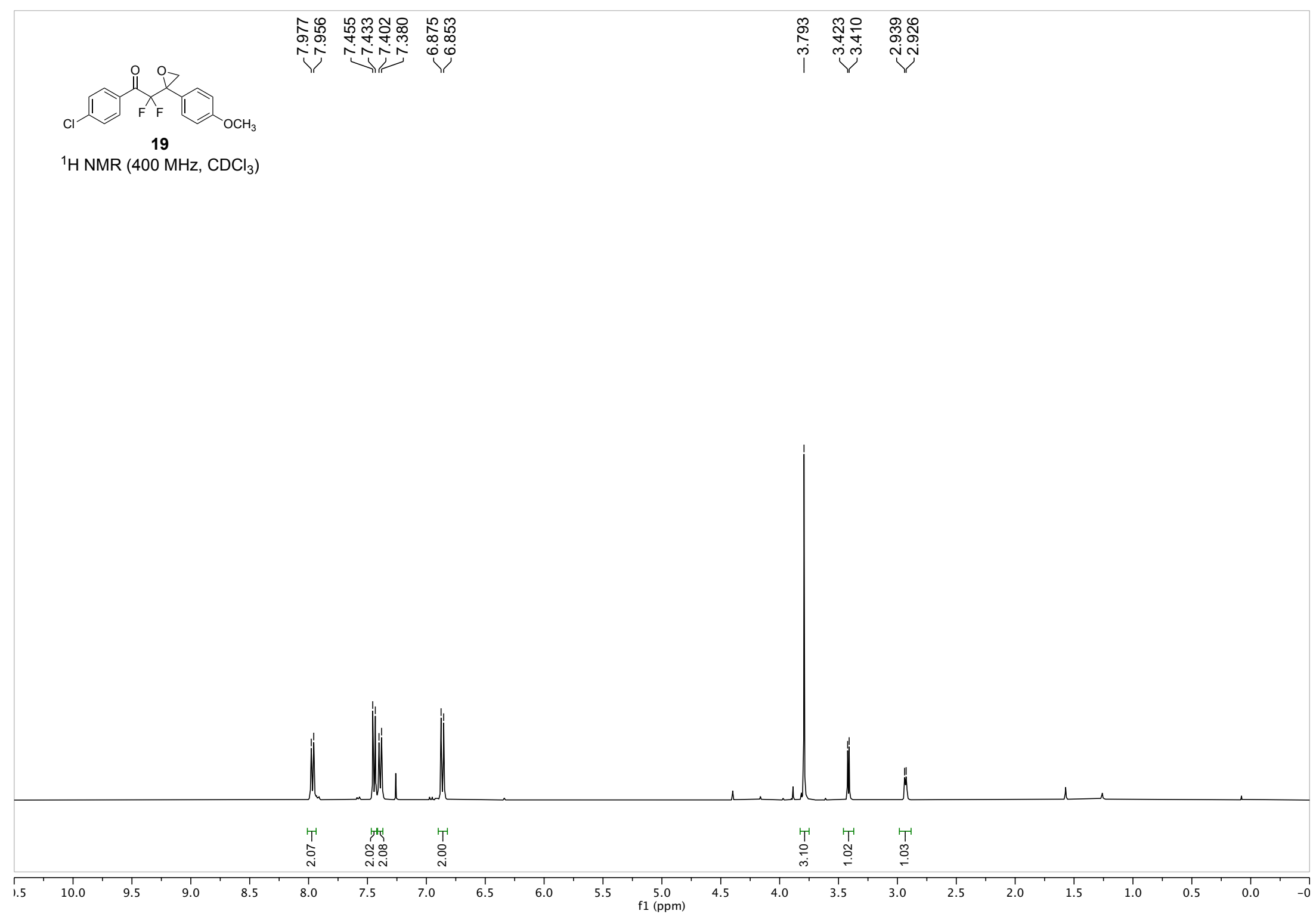




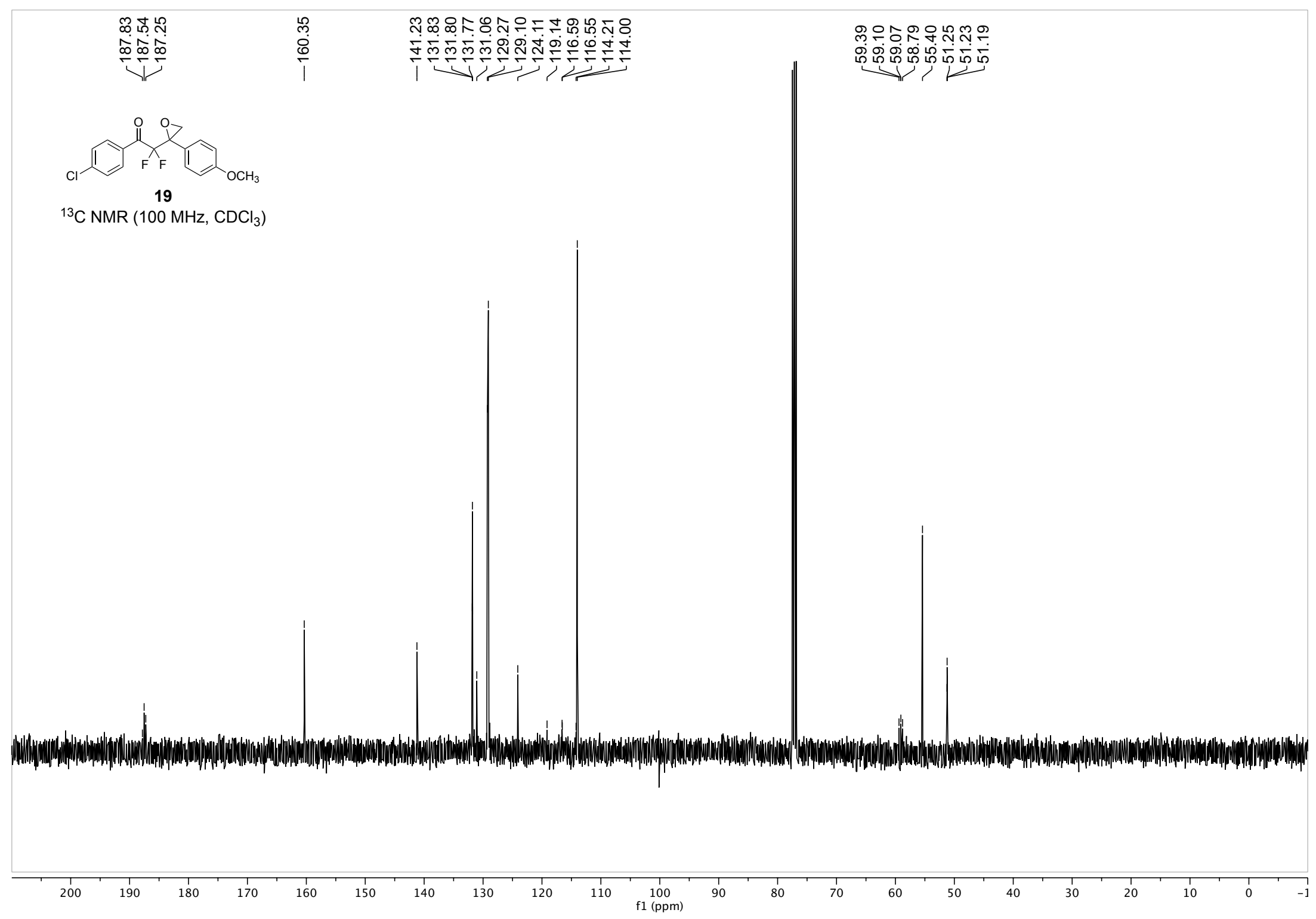




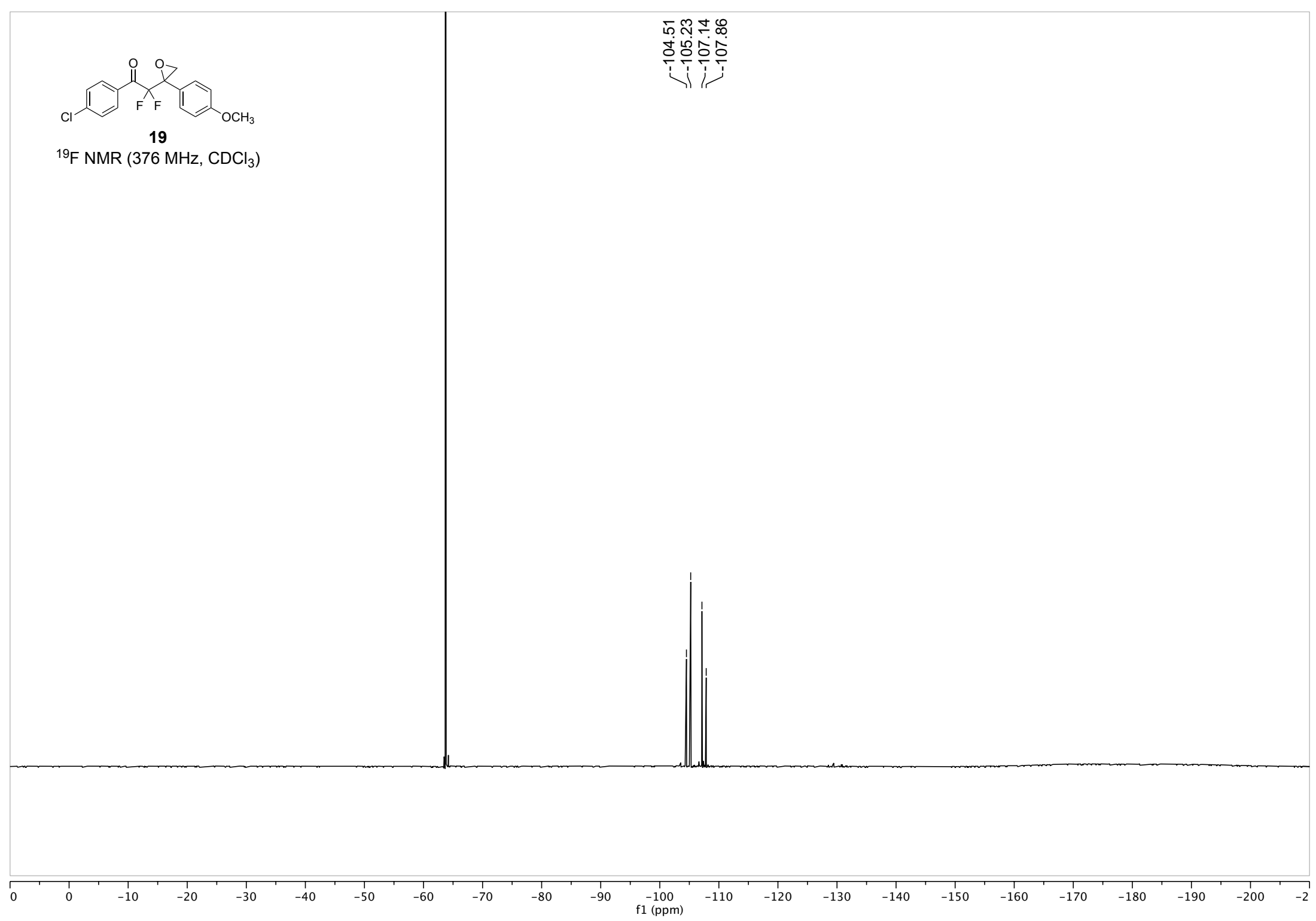




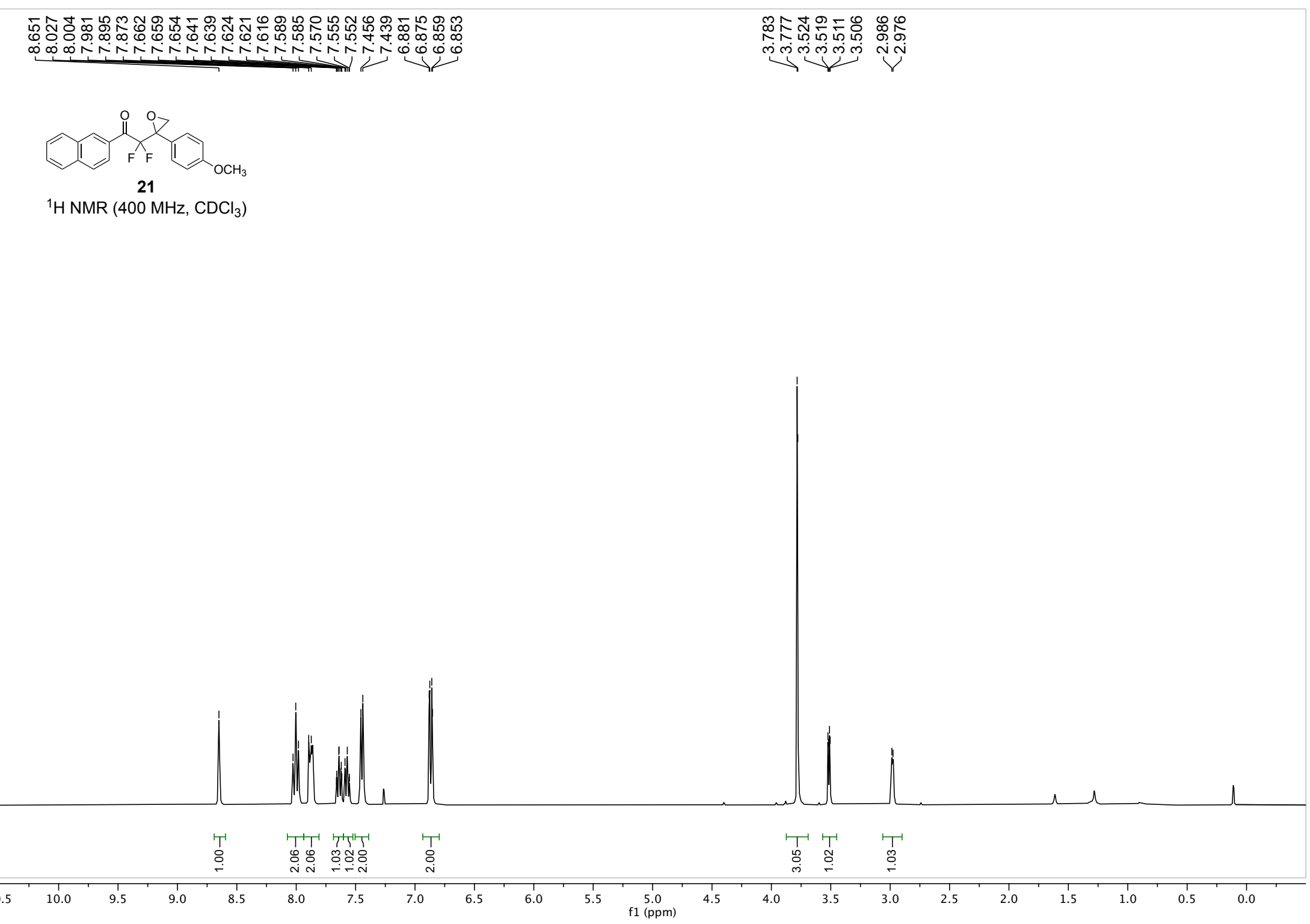




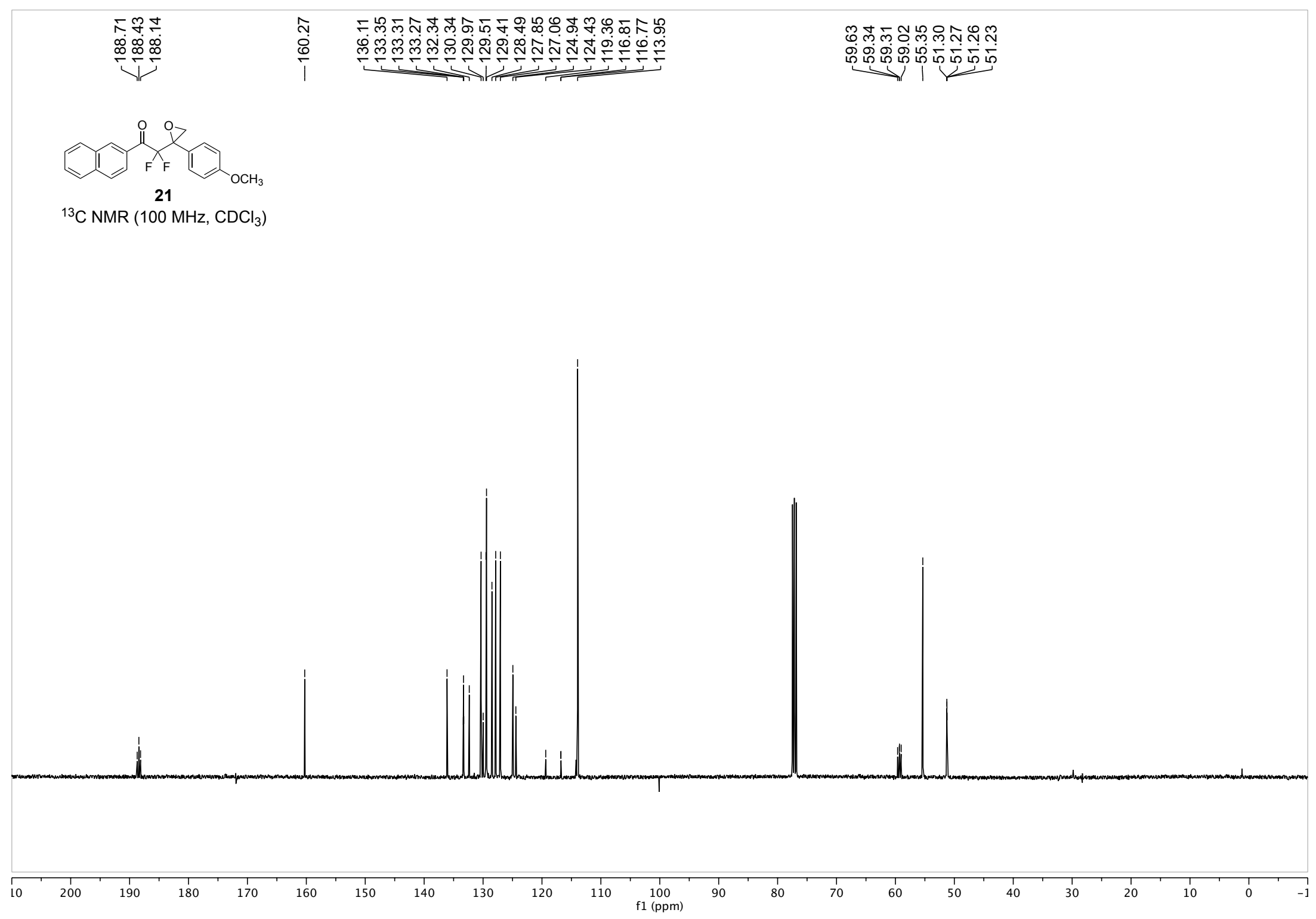




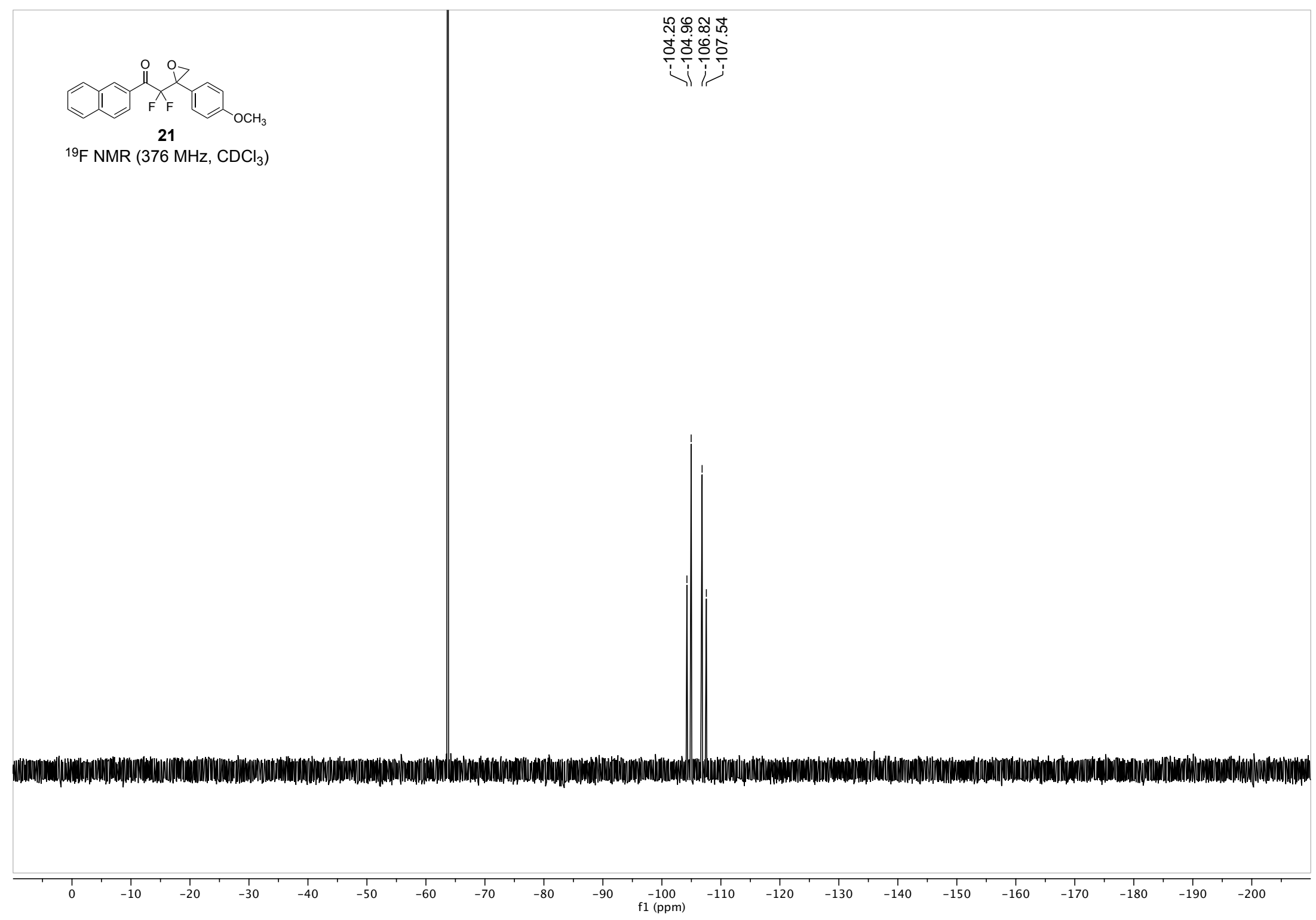




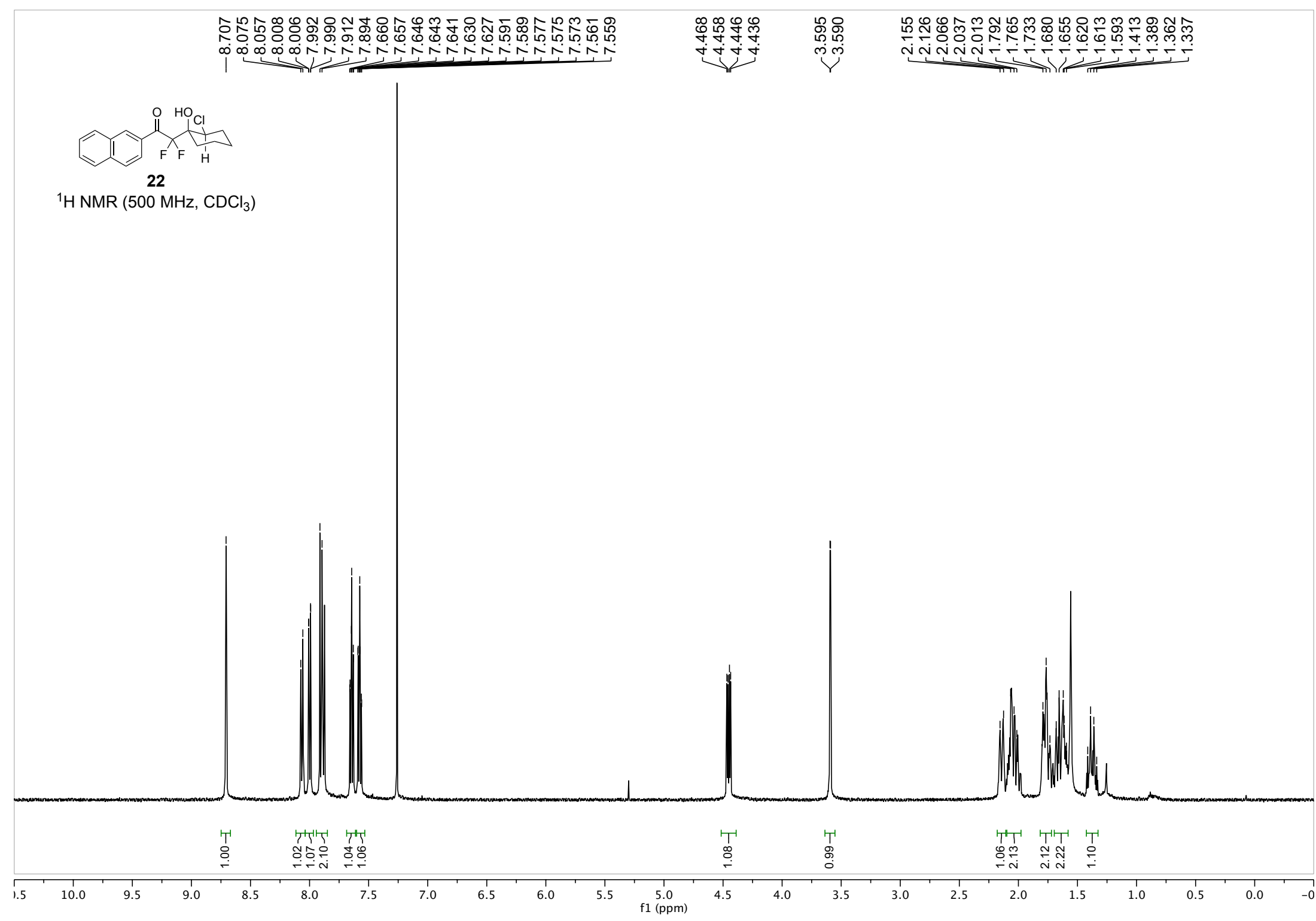



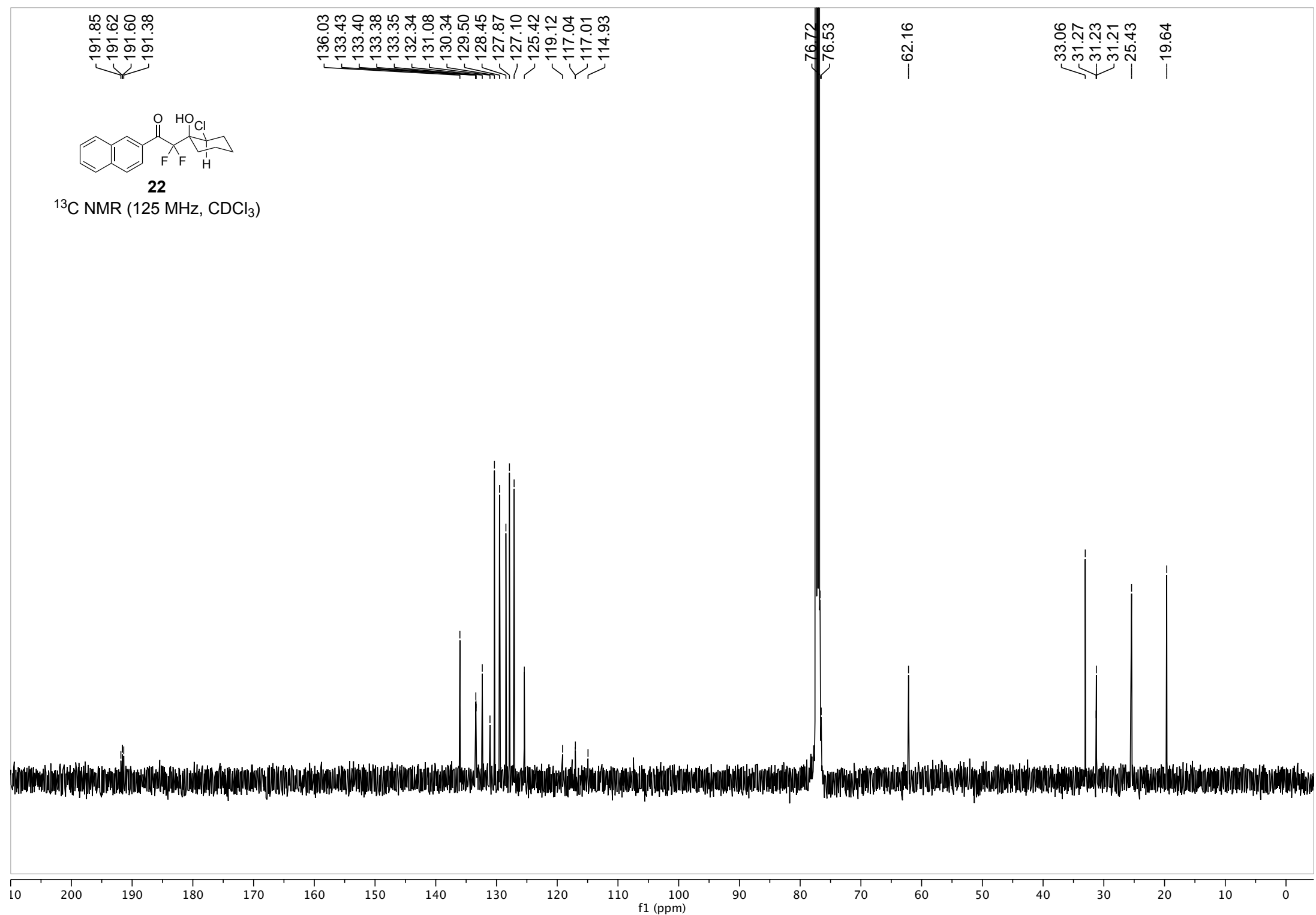


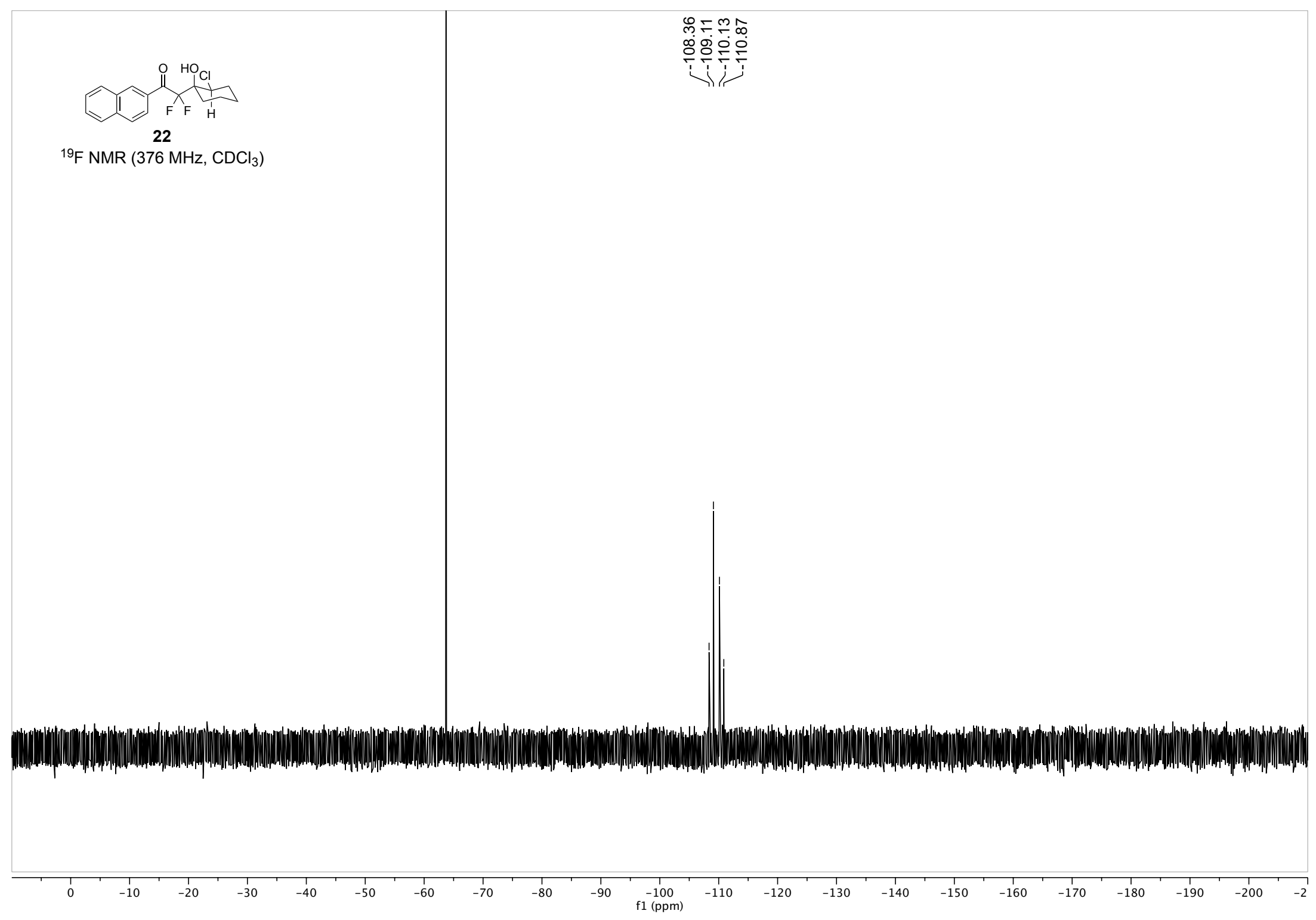




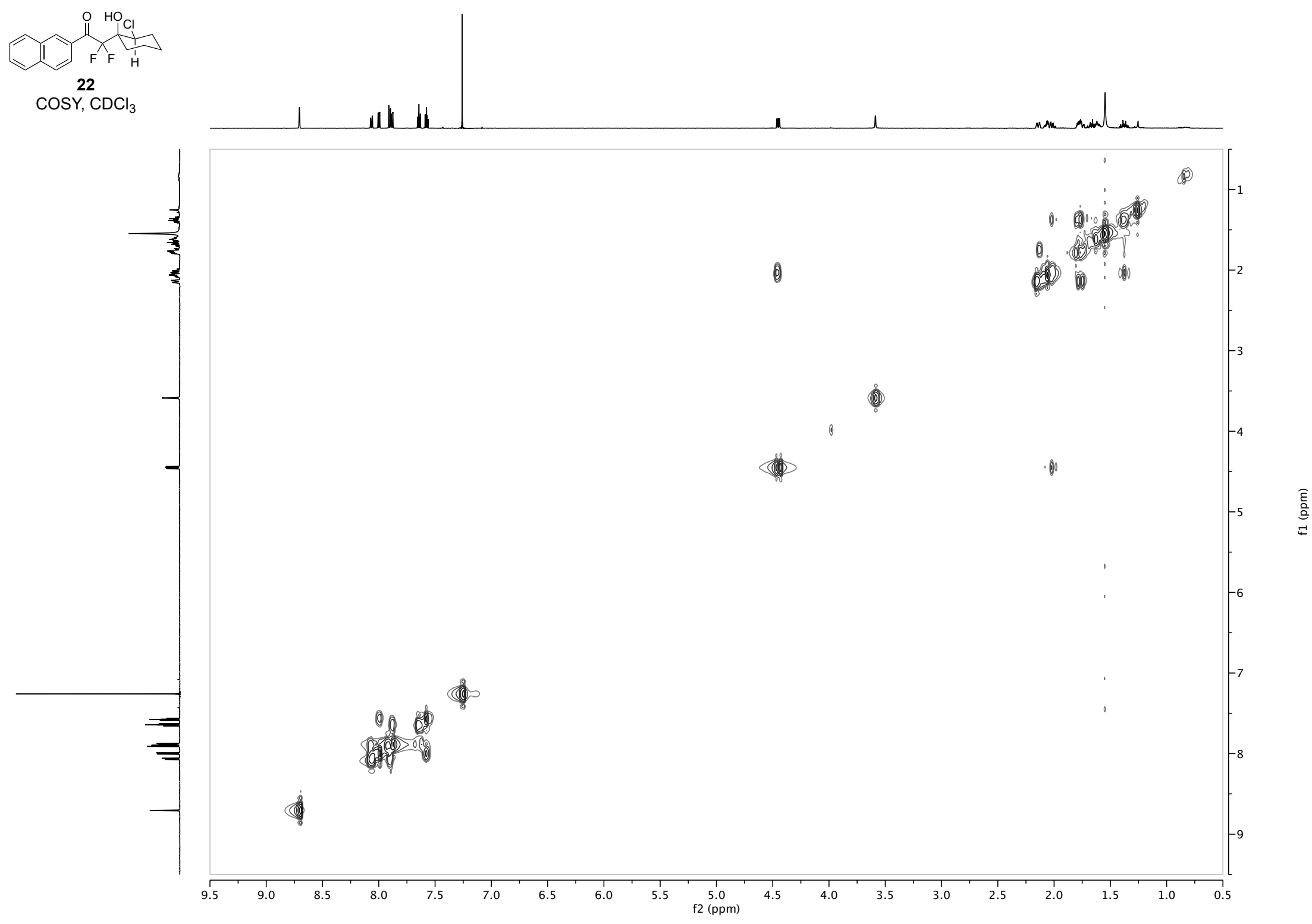

के 


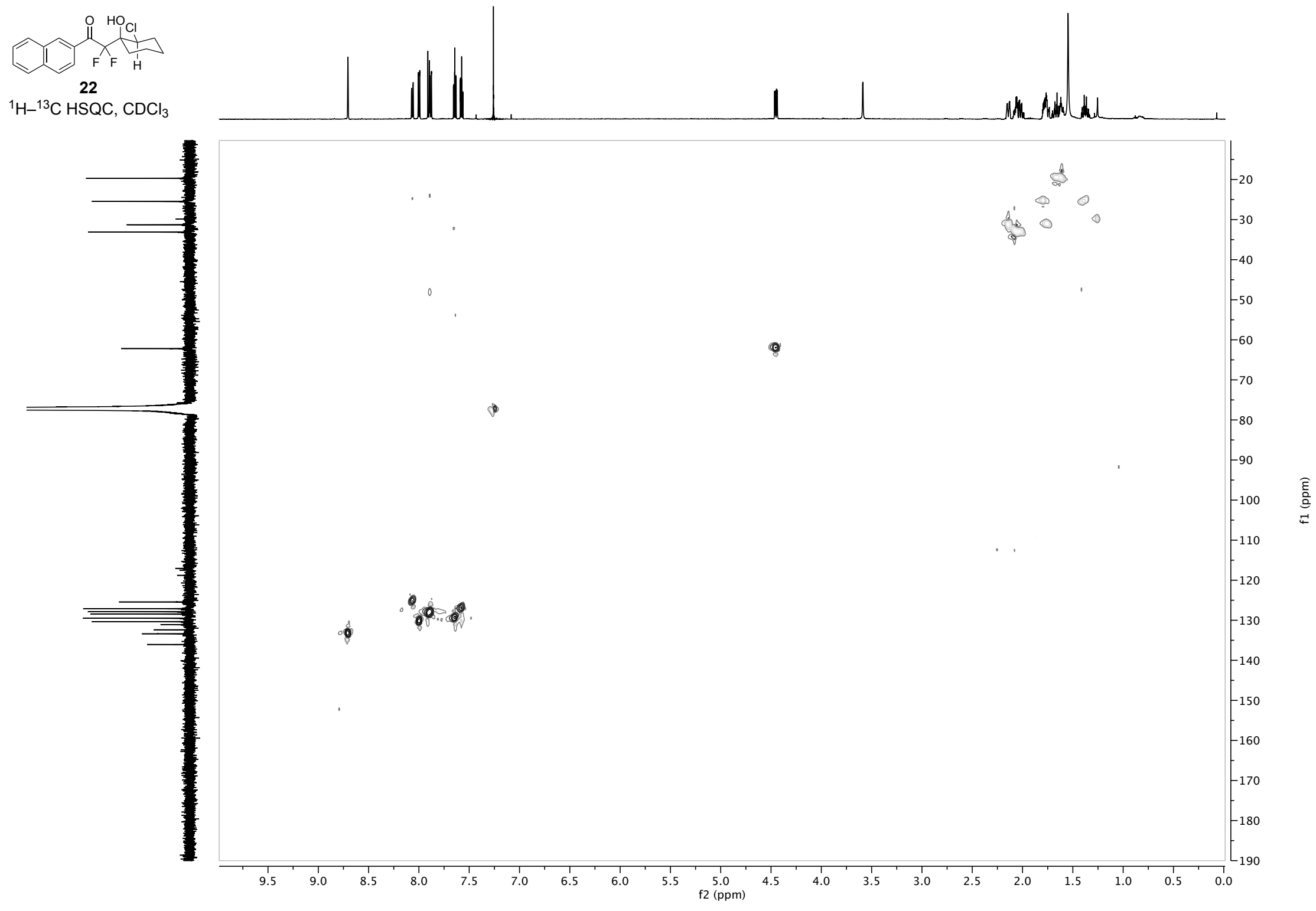




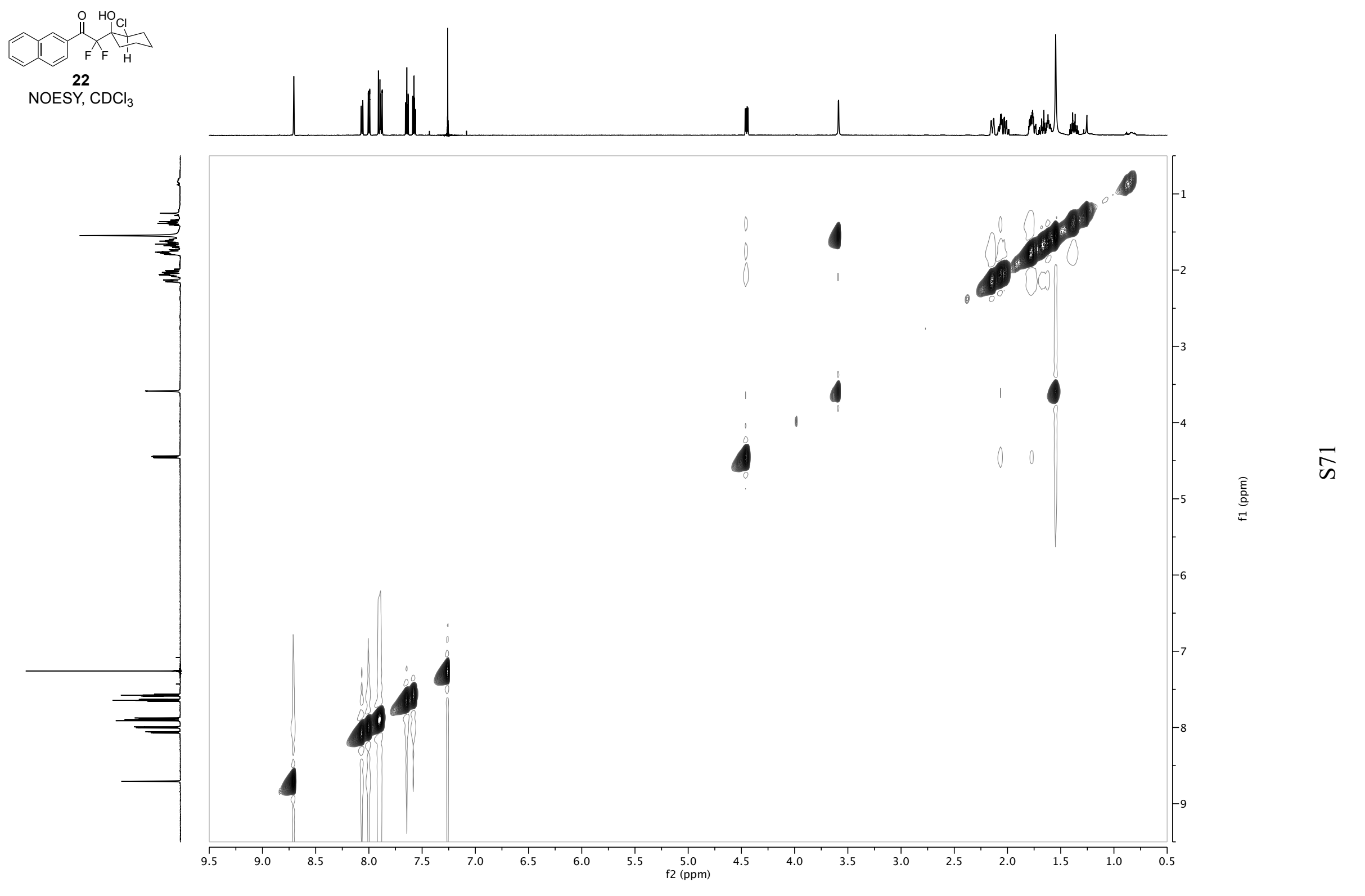




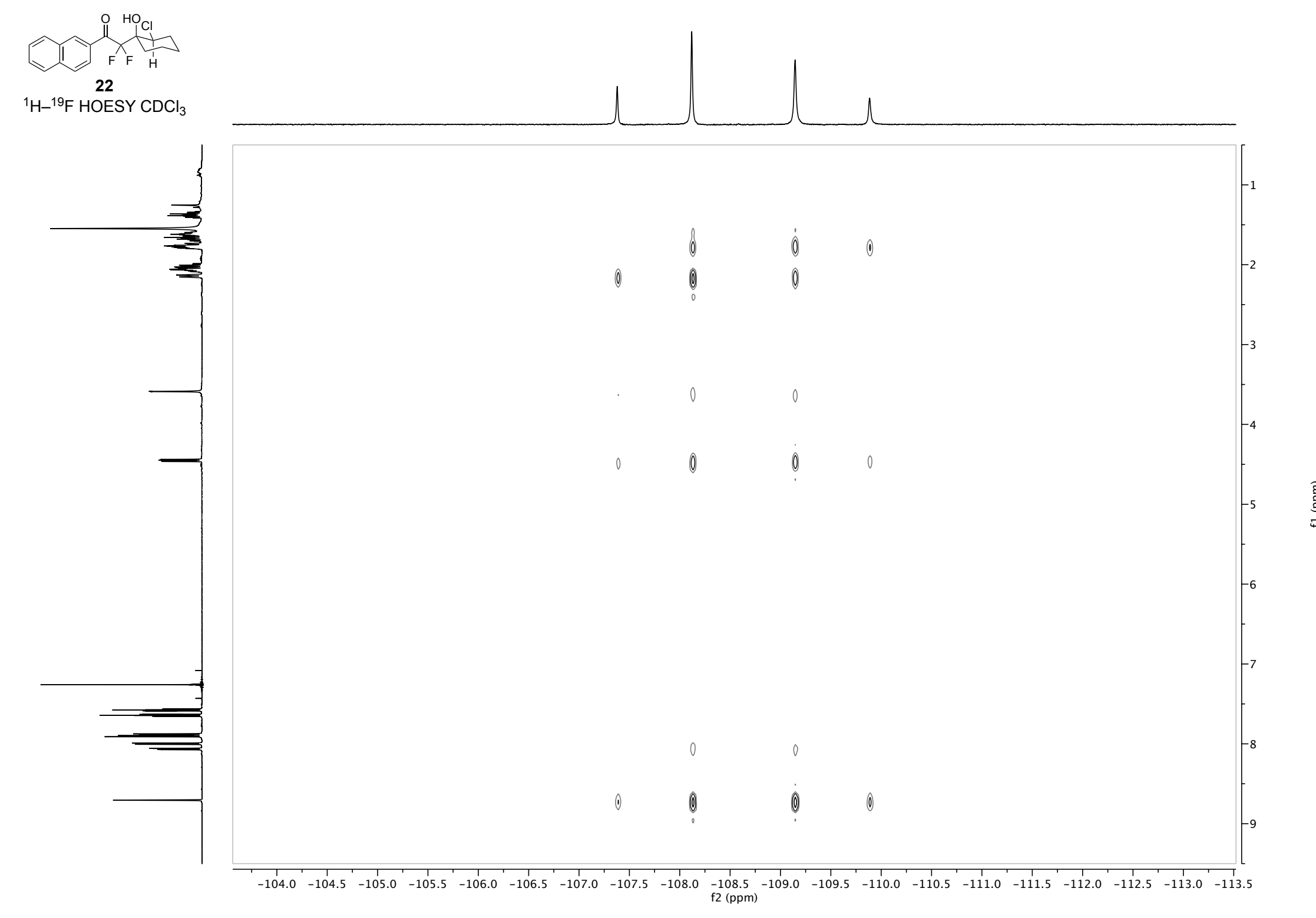




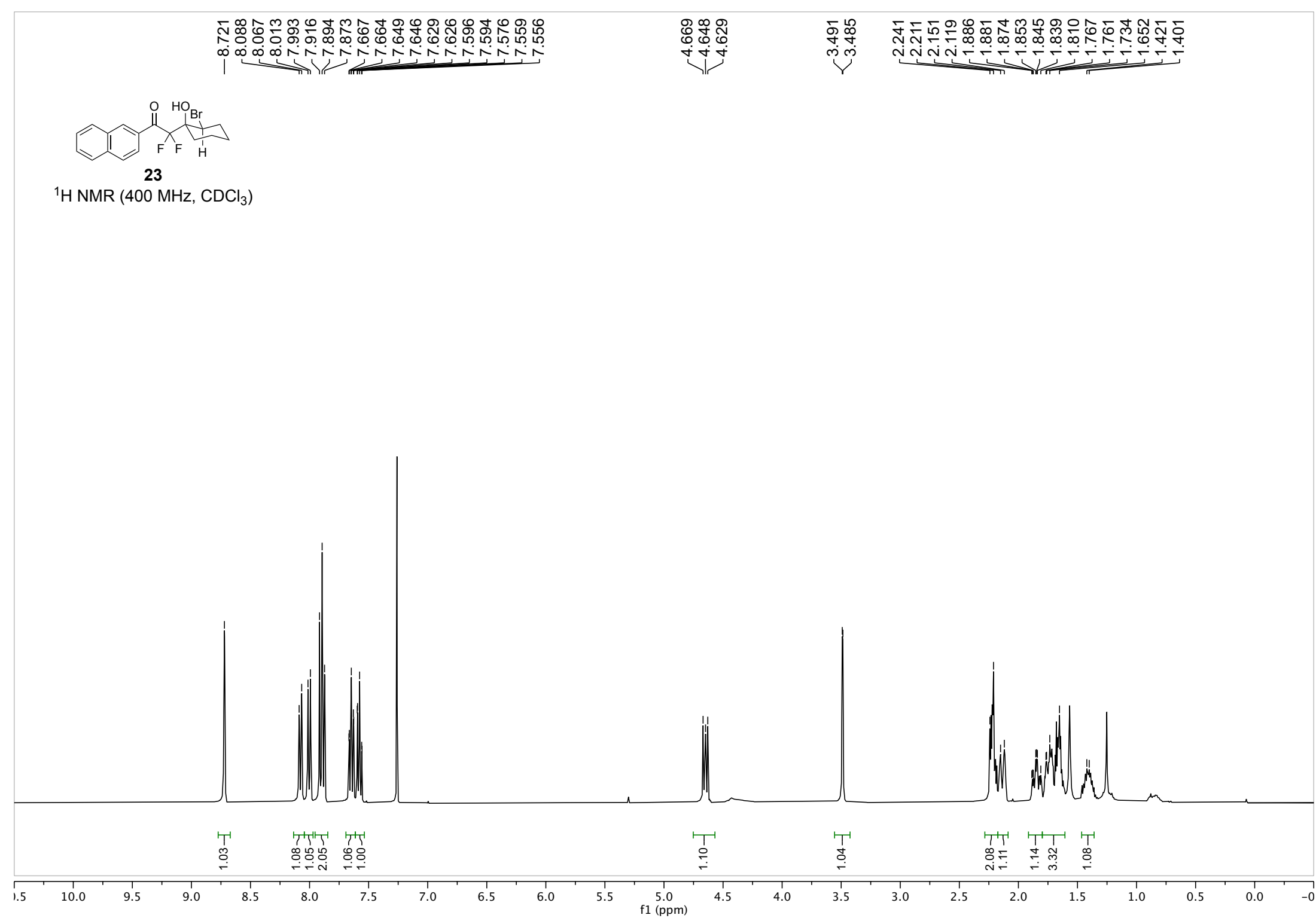




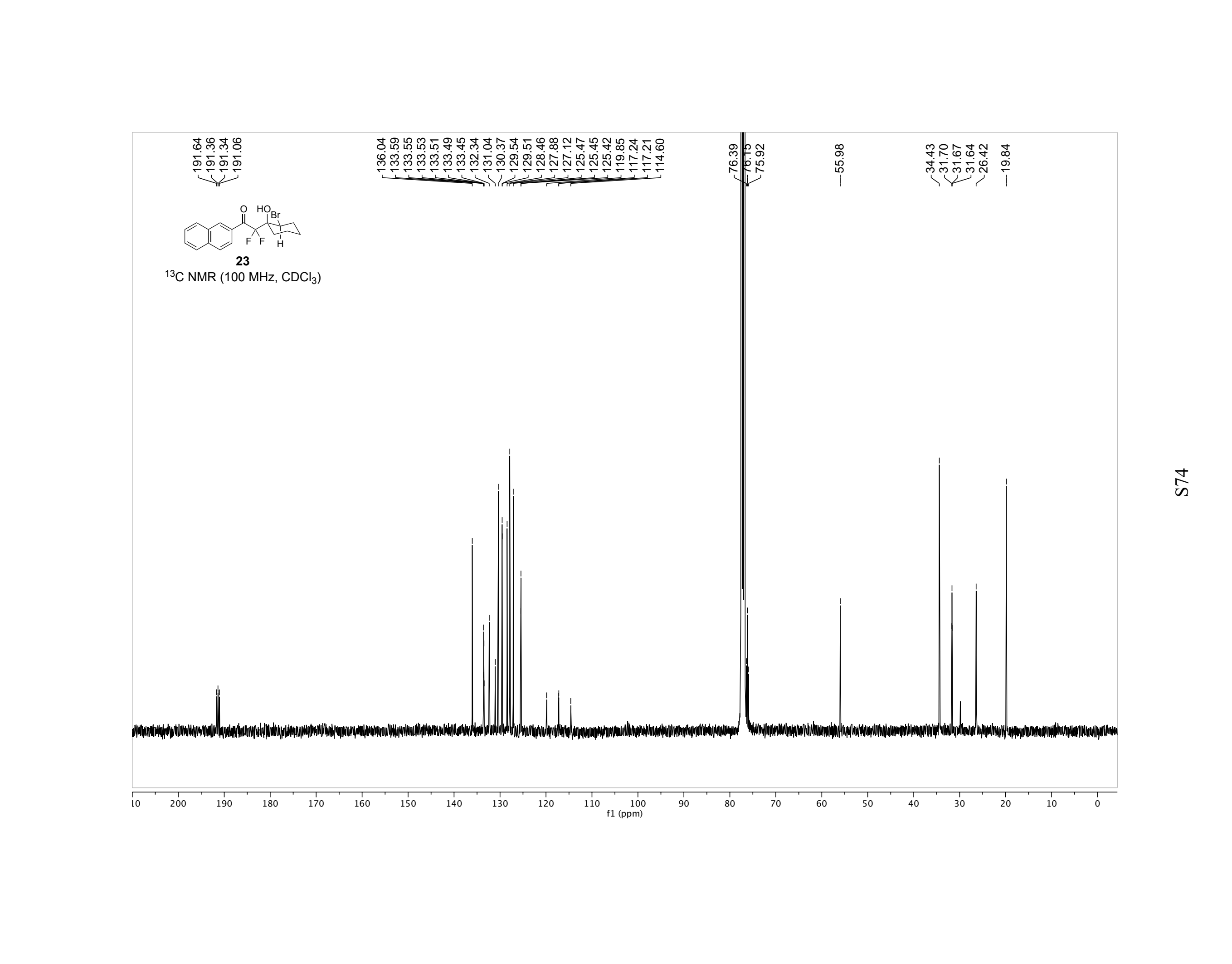



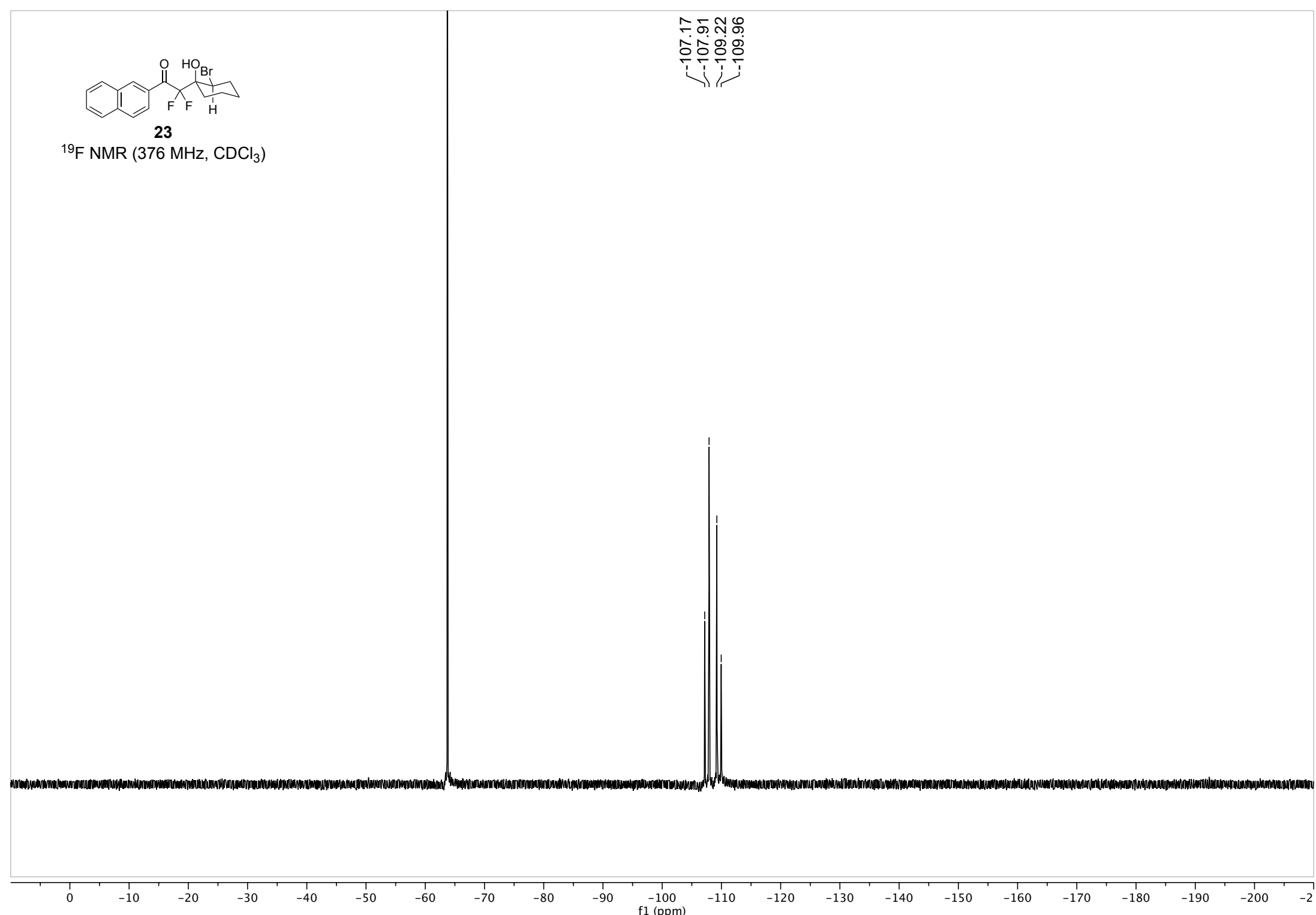


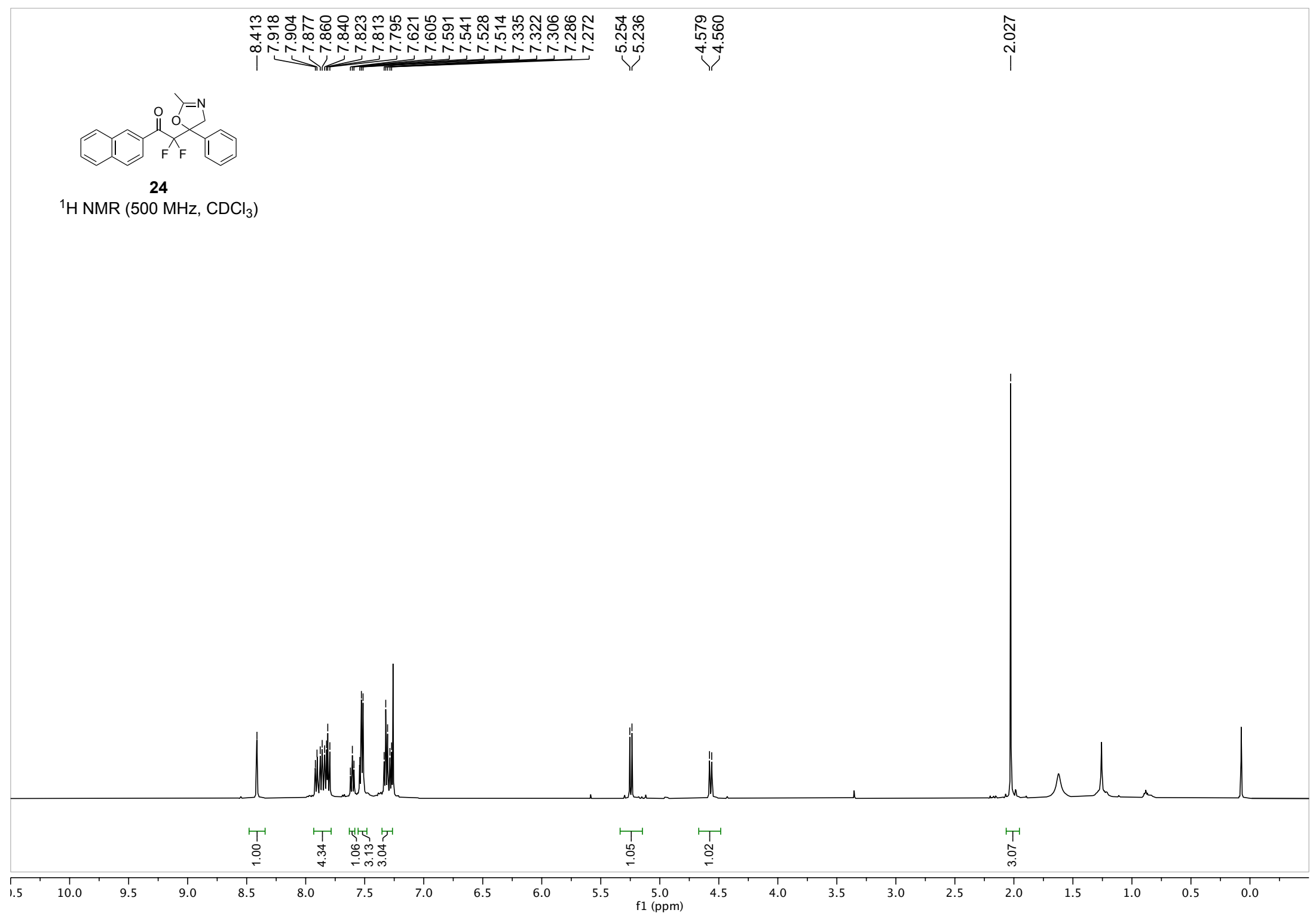




$$
\text { il }
$$



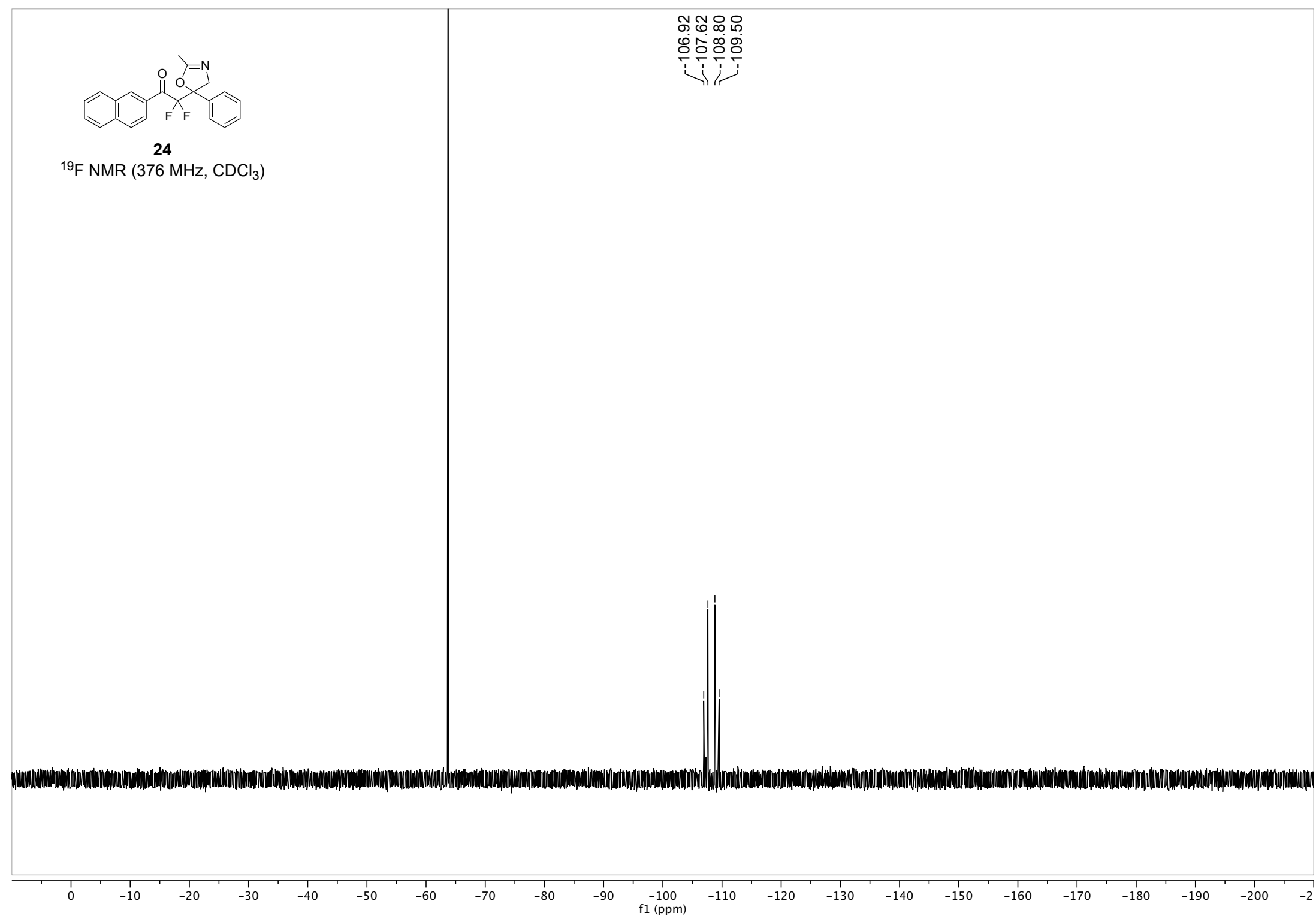


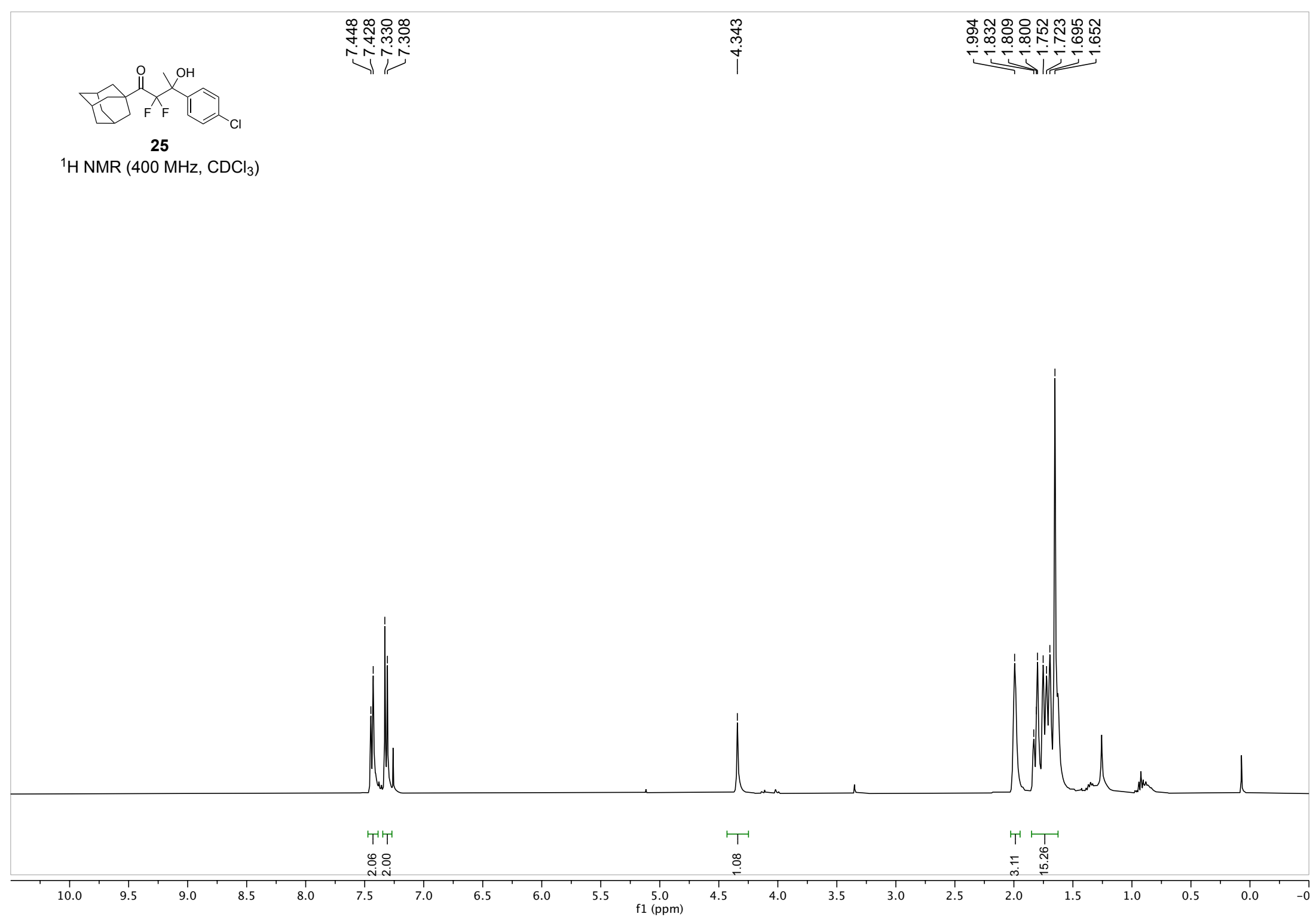




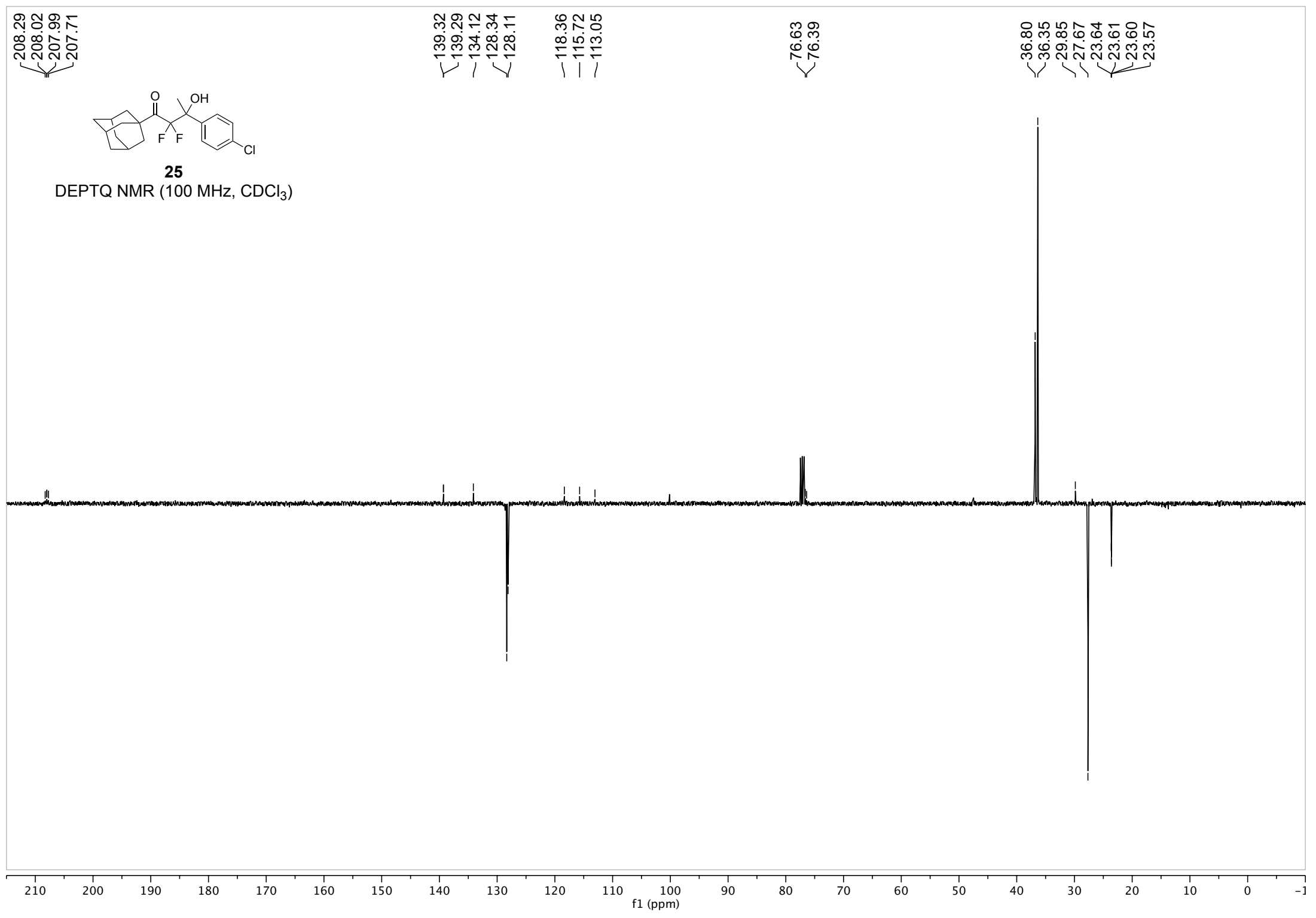

$\stackrel{\infty}{\infty}$ 


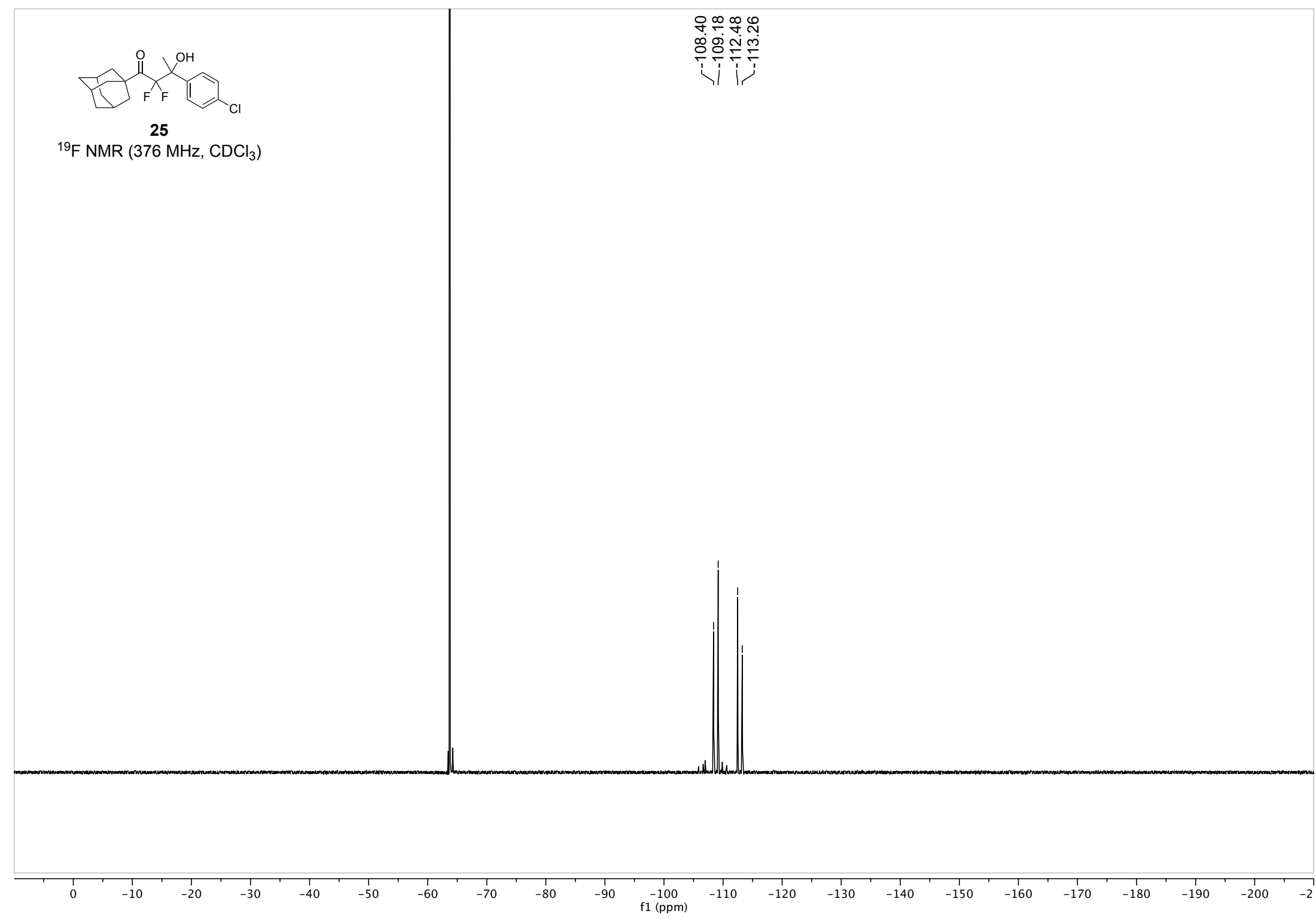

